\title{
Stereoselective Synthesis of D-Desosamine and Related Glycals via Tungsten-Catalyzed Alkynol Cycloisomerization
}

\author{
Mary H. Davidson and Frank E. McDonald* \\ Department of Chemistry, Emory University, Atlanta, GA 30322
}

\section{Supporting Information}

Experimental details and procedures for compounds 2 - 18;

${ }^{1} \mathrm{H}$ and ${ }^{13} \mathrm{C}$ NMR spectra for compounds $( \pm)-3 \mathbf{a},( \pm)-5 \mathbf{a},( \pm)-5 \mathbf{b},( \pm)-6 \mathbf{a},( \pm)-6 \mathbf{b},( \pm)-7 \mathbf{a}$, $( \pm)-7 b,( \pm)-8 a,( \pm)-8 b,( \pm)-13 a,( \pm)-14 a,( \pm)-14 b,( \pm)-15 a, 16,18$

General: ${ }^{1} \mathrm{H}$ NMR and ${ }^{13} \mathrm{C}$ NMR spectra were recorded on an Inova-400 spectrometer (400 MHz for ${ }^{1} \mathrm{H}, 100 \mathrm{MHz}$ for ${ }^{13} \mathrm{C}$ ) or an Inova-600 spectrometer $\left(600 \mathrm{MHz}\right.$ for ${ }^{1} \mathrm{H}, 150$ $\mathrm{MHz}$ for ${ }^{13} \mathrm{C}$ ). NMR spectra were recorded on solutions in deuterated chloroform $\left(\mathrm{CDCl}_{3}\right)$, with residual chloroform ( $\delta 7.27 \mathrm{ppm}$ for ${ }^{1} \mathrm{H} \mathrm{NMR}$ and $\delta 77.23 \mathrm{ppm}$ for ${ }^{13} \mathrm{C}$ NMR) taken as the internal standard, and were reported in parts per million (ppm). Abbreviations for signal coupling are as follows: $s$, singlet; $d$, doublet; $t$, triplet; $q$, quartet; m. multiplet. IR spectra were collected on a Mattson Genesis II FT-IR spectrometer as neat films. Mass spectra (low resoluion and high resolution FAB) were recorded on a VG 70-S Nier Johason Mass Spectrometer. Elemental analyses were performed by Atlantic Microlab Inc, P. O. Box 2288, Norcross, Georgia. Analytical Thin Layer Chromatography (TLC) was performed on precoated glass backed plates purchased from Whatman (silica gel $60 \quad \mathrm{~F}_{254} ; 0.25 \mathrm{~mm}$ thickness). Flash Chromatography was carried out with silica gel 60 (230-400 mesh AASTM) from EM Science. All reactions were carried out with anhydrous solvents in oven-dried or flamedried and nitrogen- or argon-charged glassware. All anhydrous solvents except as mentioned were freshly distilled. All solvents used in workup extraction procedures and chromatography were used as received from commercial suppliers without prior purification. During reaction workup, the reaction mixture was usually diluted to three times the original volume, and washed with equal volume of water or aqueous solutions as needed. All reagents were purchased from Aldrich or Strem Chemicals. 


\section{5-(tert-Butyldimethylsilanyloxy)-1-(trimethylsilyl)hex-1-yn-3-ol ( \pm )-2a and ( \pm )-2b:}

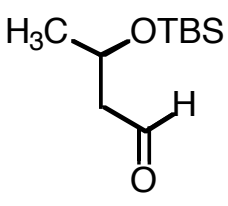

$( \pm)-1$
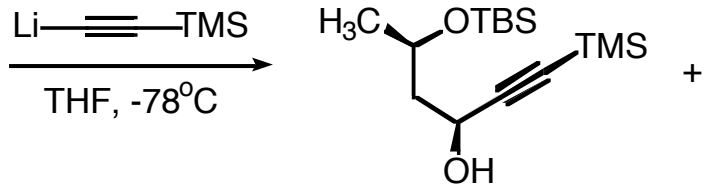

$( \pm)-2 a$

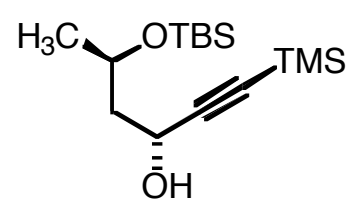

$( \pm)-2 b$

To a stirred solution of trimethylsilylacetylene $(1.473 \mathrm{~g}, 150 \mathrm{mmol})$ in THF $(30 \mathrm{~mL})$ was added a solution of $n$-butyllithium (2.5M in THF, $56 \mathrm{~mL}, 140 \mathrm{mmol}$ ) at $0{ }^{\circ} \mathrm{C}$ over $40 \mathrm{~min}$. The solution was stirred $30 \mathrm{~min}$, then cannulated into a solution of racemic aldehyde $( \pm)-1^{11}(20.24 \mathrm{~g}, 100 \mathrm{mmol})$ in THF $(120 \mathrm{~mL})$ at $-78{ }^{\circ} \mathrm{C}$. The solution was stirred for 90 min at $-78{ }^{\circ} \mathrm{C}$, then brought to $0{ }^{\circ} \mathrm{C}$ and quenched with a saturated solution of $\mathrm{NH}_{4} \mathrm{Cl}$ $(400 \mathrm{~mL})$. The organic phase was separated and the aqueous layer was extracted with ether $(3 \times 100 \mathrm{~mL})$. The organic layers were combined, dried over $\mathrm{Na}_{2} \mathrm{SO}_{4}$, and evaporated under reduced pressure. The residual oil was purified by column chromatography on silica gel (pet-ether:EtOAc $=98: 2$ ) to yield one diastereomer $(\mathbf{\pm})$-2a as a colorless oil $\left(9.3 \mathrm{~g}, 31 \%\right.$ yield). $R_{\mathrm{f}} 0.38$ (pet-ether:EtOAc $=97: 3$ ). IR (neat film) $3429,2958,2930,2898,2858,1472,1376,1252,1143,1090,1003 \mathrm{~cm}^{-1} ;{ }^{1} \mathrm{H}$ NMR $(600$ $\left.\mathrm{MHz}, \mathrm{CDCl}_{3}\right) \delta 4.59$ (ddd, $J=6.3,6.3,6 \mathrm{~Hz}, 1 \mathrm{H}$ ), 4.31 (qdd, $J=6.6,6.3,6 \mathrm{~Hz}, 1 \mathrm{H}$ ), 3.52 (d, $J=6 \mathrm{~Hz} .1 \mathrm{H}$ ), 1.83 (ddd, $J=6.6,6.3,6.3 \mathrm{~Hz}, 2 \mathrm{H}$ ), 1.20 (d, $J=6.6 \mathrm{~Hz}, 3 \mathrm{H}$ ), 0.90 (s, $9 \mathrm{H}), 0.17(\mathrm{~s}, 9 \mathrm{H}), 0.12(\mathrm{~d}, J=16.2 \mathrm{~Hz}, 6 \mathrm{H}) ;{ }^{13} \mathrm{C} \mathrm{NMR}\left(150 \mathrm{MHz}, \mathrm{CDCl}_{3}\right) \delta 106.89$, 89.07, 67.29, 60.93, 45.04, 26.03, 23.79, 18.12, 0.12, -3.98, -4.59; HRMS (FAB ${ }^{+}$) Calcd. for $\mathrm{C}_{15} \mathrm{H}_{32} \mathrm{O}_{2} \mathrm{Si}_{2} \mathrm{Li}\left[(\mathrm{M}+\mathrm{Li})^{+}\right]$307.2101, found 307.2102. Anal. Calcd. for $\mathrm{C}_{15} \mathrm{H}_{32} \mathrm{O}_{2} \mathrm{Si}_{2}$ : C, 59.94; $\mathrm{H}, 10.73$; Found: $\mathrm{C}, 60.01 ; \mathrm{H}, 10.88$. Further elution (petether:EtOAc $=98: 2)$ provided the other diastereomer $(\mathbf{(})-\mathbf{2 b}$ as a colorless oil $(14.8 \mathrm{~g}$, $49 \%$ yield). $R_{f} 0.32$ (pet-ether:EtOAc $=97: 3$ ). IR (neat film) 3403, 2959, 2930, 2897, 2858, 1472, 1373, 1252, 1143, 1091, $1002 \mathrm{~cm}^{-1} ;{ }^{1} \mathrm{H}$ NMR $\left(600 \mathrm{MHz}, \mathrm{CDCl}_{3}\right) \delta 4.53$ (ddd, $J=7.2,6.6,3.6 \mathrm{~Hz}, 1 \mathrm{H}$ ), 4.06 (qdd, $J=9.6,6.6,3.6 \mathrm{~Hz}, 1 \mathrm{H}$ ), 2.79 (d, $J=3.6 \mathrm{~Hz}$, 1H), 1.91 (ddd, $J=13.8,9.6,7.2 \mathrm{~Hz}, 1 \mathrm{H}$ ), 1.76 (ddd, $J=13.8,5.4,3.6 \mathrm{~Hz}, 1 \mathrm{H}$ ), 1.20 (d, $J=6.6 \mathrm{~Hz}, 3 \mathrm{H}), 0.88(\mathrm{~s}, 9 \mathrm{H}), 0.17(\mathrm{~s}, 9 \mathrm{H}), 0.09(\mathrm{~d}, J=5.4 \mathrm{~Hz}, 6 \mathrm{H}) ;{ }^{13} \mathrm{C}$ NMR $(150 \mathrm{MHz}$, $\left.\mathrm{CDCl}_{3}\right) \delta$ 106.63, 89.60, 67.92, 62.00, 46.89, 26.01, 24.52, 18.12, 0.08, -3.87, -4.71; HRMS $\left(F A B^{+}\right)$Calcd. for $\mathrm{C}_{15} \mathrm{H}_{32} \mathrm{O}_{2} \mathrm{Si}_{2} \mathrm{Li}\left[(\mathrm{M}+\mathrm{Li})^{+}\right]$307.2101, found 307.2089. Anal. Calcd. for $\mathrm{C}_{15} \mathrm{H}_{32} \mathrm{O}_{2} \mathrm{Si}_{2}$ : C, 59.94; $\mathrm{H}, 10.73$; Found: C, 59.96; $\mathrm{H}, 10.97$. 
Synthesis of 5-(tert-Butyldimethylsilanyloxy)-1-(trimethylsilyl)-3-aminohex-1-yne (士)-3a:

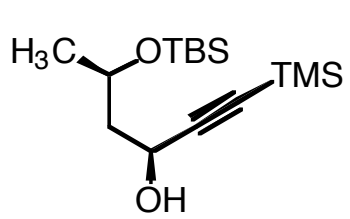

$( \pm)-2 a$
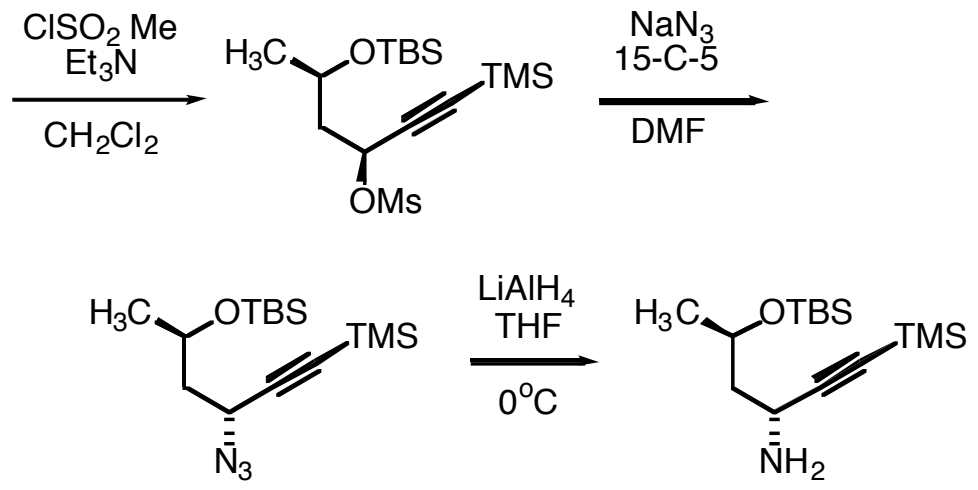

$( \pm)-3 a$

Formation of mesylate from ( $\mathbf{\pm})-\mathbf{2 a}$ : To a stirred solution of $2 \mathbf{a}(9.51 \mathrm{~g}, 31 \mathrm{mmol})$ in $\mathrm{CH}_{2} \mathrm{Cl}_{2}(155 \mathrm{~mL})$ at room temperature was added $\mathrm{Et}_{3} \mathrm{~N}(13.2 \mathrm{~mL}, 95 \mathrm{mmol})$. After stirring for $30 \mathrm{~min}$, the solution was cooled to $0{ }^{\circ} \mathrm{C}$ and $\mathrm{CISO}_{2} \mathrm{CH}_{3}(12 \mathrm{~mL}, 155 \mathrm{mmol})$ was added dropwise over $60 \mathrm{~min}$. The reaction was allowed to warm to room temperature with stirring over $5 \mathrm{~h}$, at which time it was quenched by addition of $\mathrm{NaHCO}_{3}$ $(400 \mathrm{~mL})$ and stirred for an additional $2 \mathrm{~h}$. The organic phase was separated and the aqueous layer was extracted with $\mathrm{CH}_{2} \mathrm{Cl}_{2}(3 \times 100 \mathrm{~mL})$. The combined organic layers were dried over $\mathrm{Na}_{2} \mathrm{SO}_{4}$ and evaporated under reduced pressure. The residual oil was purified by column chromatography on silica gel (pet-ether:EtOAc $=90: 1$ ) to yield mesylate as a pure oil $\left(11.10 \mathrm{~g}, 95 \%\right.$ yield). $R_{f} 0.41$ (pet-ether:EtOAc $=92: 8$ ). IR (neat film) 2958, 2931, 2899, 2857, 1472, 1366, 1253, 1178, 1144, $1099 \mathrm{~cm}^{-1} ;{ }^{1} \mathrm{H}$ NMR (400 $\left.\mathrm{MHz} \mathrm{CDCl}_{3}\right) \delta 5.25(\mathrm{dd}, J=9.2,4 \mathrm{~Hz}, 1 \mathrm{H}) ; 4.01(\mathrm{dqd}, J=9,6,3.6 \mathrm{~Hz}, 1 \mathrm{H}), 3.15(\mathrm{~s}$, $3 \mathrm{H}$ ), 2.01 (ddd $J=14.6,9.2,3.6 \mathrm{~Hz}, 1 \mathrm{H}), 1.92(\mathrm{ddd}, J=14.6,9,4 \mathrm{~Hz}, 1 \mathrm{H}), 1.18(\mathrm{~d}, J=$ $6 \mathrm{~Hz}, 3 \mathrm{H}), 0.90(\mathrm{~s}, 9 \mathrm{H}), 0.20(\mathrm{~s}, 9 \mathrm{H}), 0.10(\mathrm{~d}, J=11.6 \mathrm{~Hz}, 6 \mathrm{H}) ;{ }^{13} \mathrm{C}$ NMR $(100 \mathrm{MHz}$, $\left.\mathrm{CDCl}_{3}\right) \delta 101.17,94.53,69.90,64.22,46.04,39.74,26.10,24.14,18.17,-0.25,-3.92$, 4.61; HRMS (FAB $\left.{ }^{+}\right)$Calcd. for $\mathrm{C}_{16} \mathrm{H}_{34} \mathrm{O}_{4} \mathrm{Si}_{2} \mathrm{SLi}\left[(\mathrm{M}+\mathrm{Li})^{+}\right]$385.1876, found 385.1864. Anal. Calcd. for $\mathrm{C}_{16} \mathrm{H}_{34} \mathrm{O}_{4} \mathrm{Si}_{2} \mathrm{~S}$ : C, 50.75; H, 9.05; S, 8.47; Found: C, 50.87; H, 9.07; S, 8.22 . 
Formation of azide from the mesylate derivative of ( $\mathbf{(})-2 \mathrm{a}$ : To a stirred solution of the above-described mesylate $(11.0 \mathrm{~g}, 29 \mathrm{mmol})$ in DMSO $(29 \mathrm{~mL})$ was added 15-Crown-5 $(5.8 \mathrm{~mL}, 29 \mathrm{mmol})$ and $\mathrm{NaN}_{3}(9.45 \mathrm{~g}, 145 \mathrm{mmol})$ at room temperature. The solution was stirred at room temperature for $6 \mathrm{~h}$, after which time the reaction vessel was fitted with a reflux condenser and heated to $60{ }^{\circ} \mathrm{C}$ for $2 \mathrm{~h}$. The reaction was quenched with $\mathrm{H}_{2} \mathrm{O}(50 \mathrm{~mL})$. The organic phase was separated and the aqueous layer was extracted with EtOAc $(3 \times 50 \mathrm{~mL})$. The combined organic layers were dried over $\mathrm{Na}_{2} \mathrm{SO}_{4}$ and evaporated under reduced pressure. The residual oil was purified by column chromatography on silica gel (pet-ether:EtOAc $=95: 5)$ to yield azide as a pure oil (8.75 g, $92 \%$ yield). $R_{f} 0.43$ (pet-ether:EtOAc $=95: 5$ ). IR (neat film) 2958, 2930, 2898, 2858, 2107, 1472, 1375, 1252, 1144, 1094, $1057 \mathrm{~cm}^{-1} ;{ }^{1} \mathrm{H}$ NMR (400 MHz, CDCl $) \delta 4.19$ (dd, $J=10.2,5.2 \mathrm{~Hz}, 1 \mathrm{H}$ ), 4.02 (dqd, $J=9.6,6,3.2 \mathrm{~Hz}, 1 \mathrm{H}$ ), Hz, 1H), 1.81 (ddd, $J=14.2$, 9.6, $5.2 \mathrm{~Hz}, 1 \mathrm{H}$ ), 1.67 (ddd, $J=14.2,10.2,3.2 \mathrm{~Hz}, 1 \mathrm{H}$ ), 1.18 (d, $J=9 \mathrm{~Hz}, 3 \mathrm{H}$ ), 0.89 (s, $9 \mathrm{H}), 0.20(\mathrm{~s}, 9 \mathrm{H}), 0.08(\mathrm{~s}, 6 \mathrm{H}) ;{ }^{13} \mathrm{C}$ NMR $\left(100 \mathrm{MHz}, \mathrm{CDCl}_{3}\right) \delta$ 100.73, 92.72, 65.84, $51.55,44.84,26.06,24.28,18.21,0.88,-4.01,-4.69$.

Reduction of the azide to amine ( $\mathbf{\pm})-\mathbf{3 a}$ : To a stirred solution of the above-described azide $(3.20 \mathrm{~g}, 10 \mathrm{mmol})$ in THF (18 mL) was added LAH (1M in THF, $15.1 \mathrm{~mL}, 0.58 \mathrm{~g}$, $17.1 \mathrm{mmol}$ ) at $0{ }^{\circ} \mathrm{C}$ dropwise, and the solution was stirred for $1 \mathrm{~h}$ at $0^{\circ} \mathrm{C}$. The reaction was carefully quenched stepwise with $\mathrm{H}_{2} \mathrm{O}(0.6 \mathrm{~mL}), \mathrm{NaOH}(15 \%$ soln, $1.12 \mathrm{~mL})$, then $\mathrm{H}_{2} \mathrm{O}(1.8 \mathrm{~mL})$ at $0{ }^{\circ} \mathrm{C}$ after which it was stirred $30 \mathrm{~min}$ at room temperare. The reaction mixture was dried over $\mathrm{Na}_{2} \mathrm{SO}_{4}$, filtered through celite, and evaporated under reduced pressure. The residual oil was purified by column chromatography on silica gel (petether:EtOAc $=90: 10)$ to yield $( \pm)-3 a$ as a pure oil $\left(2.9 \mathrm{~g}, 96 \%\right.$ yield). $R_{f} 0.27$ (petether:EtOAc = 8:2). IR (neat film) 3374, 3286, 2958, 2929, 2899, 2857, 2164, 1599, 1472, 1463, 1373, 1251, 1145, 1073, $997 \mathrm{~cm}^{-1} ;{ }^{1} \mathrm{H}$ NMR $\left(600 \mathrm{MHz}, \mathrm{CDCl}_{3}\right) \delta 4.02$ (dqd, $J=9.3,6.6,3 \mathrm{~Hz}, 1 \mathrm{H}$ ), 3.70 (dd, $J=10.2,4.8 . \mathrm{Hz}, 1 \mathrm{H}$ ), 1.73 (ddd, $J=14,9.3,4.8 \mathrm{~Hz}$, 1H), 1.53 (ddd, $J=14,10.2,3 \mathrm{~Hz}, 1 \mathrm{H}$ ), 1.43 (brs, 2H), 1.16 (d, $J=6.6 \mathrm{~Hz}, 3 \mathrm{H}$ ), 0.88 (s, $9 \mathrm{H}), 0.15(\mathrm{~s}, 9 \mathrm{H}), 0.075(\mathrm{~d}, J=9.6 \mathrm{~Hz}, 6 \mathrm{H}) ;{ }^{13} \mathrm{C}$ NMR $\left(150 \mathrm{MHz}, \mathrm{CDCl}_{3}\right) \delta$ 110.16, 87.07, 66.56, 48.37, 42.14, 26.10, 24.57, 18.22, 0.241, -4.01, -4.62; HRMS (FAB $\left.{ }^{+}\right)$ Calcd. for $\mathrm{C}_{15} \mathrm{H}_{34} \mathrm{ONSi}_{2}\left[(\mathrm{M}+\mathrm{H})^{+}\right] 300.2179$, found 300.2166 . 
Synthesis of diastereomer $( \pm)-3 b$ :

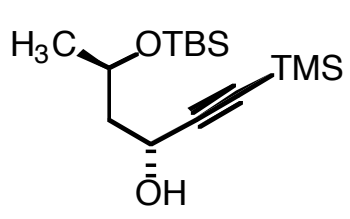

$( \pm)-2 b$
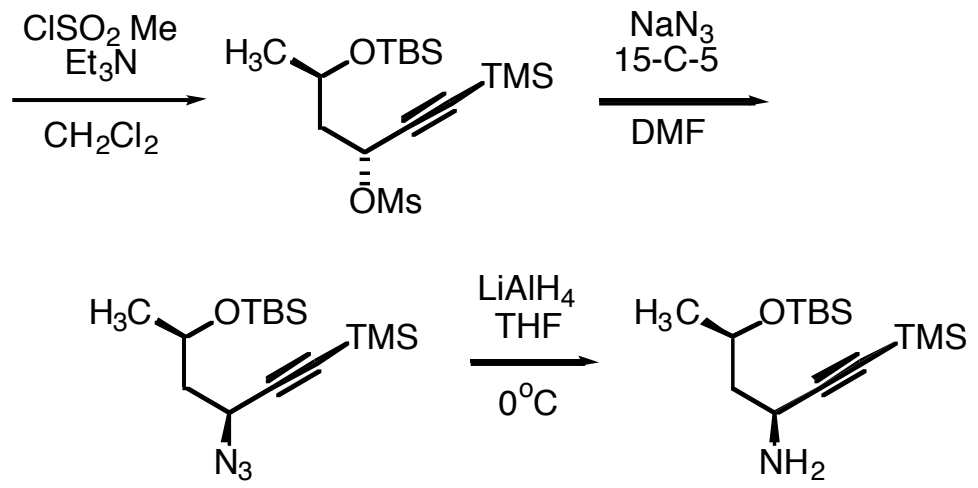

$( \pm)-3 b$

The procedure as described above from $\mathbf{2 a}$ was followed with $\mathbf{2 b}(11 \mathrm{~g}, 37 \mathrm{mmol})$, $\mathrm{CH}_{2} \mathrm{Cl}_{2}(185 \mathrm{~mL}), \mathrm{Et}_{3} \mathrm{~N}(15 \mathrm{~mL}, 110 \mathrm{mmol})$, and $\mathrm{ClSO}_{2} \mathrm{CH}_{3}(14 \mathrm{~mL}, 185 \mathrm{mmol})$, and purification by column chromatography on silica gel (pet-ether:EtOAc $=90: 1$ ) gave the mesylate as a pure oil $\left(13.90 \mathrm{~g}, 99 \%\right.$ yield). $R_{f} 0.36$ (pet-ether:EtOAc $=92: 8$ ). IR (neat film) 2958, 2931, 2898, 2858, 1472, 1464, 1369, 1253, 1179, $1076 \mathrm{~cm}^{-1} ;{ }^{1} \mathrm{H}$ NMR (400 $\left.\mathrm{MHz}, \mathrm{CDCl}_{3}\right) \delta 5.28(\mathrm{dd}, J=9.6,5.6 \mathrm{~Hz}, 1 \mathrm{H}), 4.02(\mathrm{dqd}, J=9.2,6.4,3.6 \mathrm{~Hz}, 1 \mathrm{H}), 3.11$ (s, 3H), 2.08 (ddd, $J=13.2,9.2,5.6 \mathrm{~Hz}, 1 \mathrm{H}$ ), 1.87 (ddd, $J=13.2,9.6,3.6 \mathrm{~Hz}, 1 \mathrm{H}$ ), 1.21 (d, $J=6.4 \mathrm{~Hz}, 3 \mathrm{H}), 0.89(\mathrm{~s}, 9 \mathrm{H}), 0.19(\mathrm{~s}, 9 \mathrm{H}), 0.074(\mathrm{~d}, J=4 \mathrm{~Hz}, 6 \mathrm{H}) ;{ }^{13} \mathrm{C}$ NMR $(100$ $\left.\mathrm{MHz}, \mathrm{CDCl}_{3}\right) \delta 100.61,95.18,70.93,65.26,45.18,39.40,25.98,24.10,18.14,-0.25$, -3.98, -4.78. HRMS $\left(\mathrm{FAB}^{+}\right)$Calcd. for $\mathrm{C}_{16} \mathrm{H}_{34} \mathrm{O}_{4} \mathrm{Si}_{2} \mathrm{SLi}\left[(\mathrm{M}+\mathrm{Li})^{+}\right] 385.1876$, found 385.1864 .

Conversion to the azide from the mesylate $(8.51 \mathrm{~g}, 22.4 \mathrm{mmol})$, DMSO $(25 \mathrm{~mL}) 15$ Crown-5 (4.4 mL, $22.4 \mathrm{mmol})$, and $\mathrm{NaN}_{3}(5.85 \mathrm{~g}, 90 \mathrm{mmol})$ and purification by column chromatography on silica gel (pet-ether:EtOAc $=90: 1$ ) provided the azide as a pure oil (6.45 g, 88\% yield). $R_{f} 0.35$ (pet-ether:EtOAc $=95: 5$ ). IR (neat film) 2958, 2930, 2898, 2858, 2107, 1472, 1464, 1375, 1252, 1143, 1097, $1057 \mathrm{~cm}^{-1} ;{ }^{1} \mathrm{H}$ NMR $(400 \mathrm{MHz}$, $\left.\mathrm{CDCl}_{3}\right) \delta 4.23(\mathrm{dd}, J=10,4.8 \mathrm{~Hz}, 1 \mathrm{H}), 3.95(\mathrm{dqd}, J=9.6,6,3.6 \mathrm{~Hz}, 1 \mathrm{H}), 1.83-1.67(\mathrm{~m}$, 2H), $1.15(\mathrm{~d}, J=6 \mathrm{~Hz}, 3 \mathrm{H}), 0.89(\mathrm{~s}, 9 \mathrm{H}), 0.20(\mathrm{~s}, 9 \mathrm{H}), 0.073(\mathrm{~s}, 6 \mathrm{H}) ;{ }^{13} \mathrm{C}$ NMR $(100$ $\left.\mathrm{MHz}, \mathrm{CDCl}_{3}\right) \delta 101.30,91.74,64.67,50.49,45.30,26.06,24.10,18.21,0.11,-4.12$, -4.72; Anal. Calcd. for $\mathrm{C}_{15} \mathrm{H}_{31} \mathrm{ON}_{3} \mathrm{Si}_{2}:$ C, 55.34; $\mathrm{H}, 9.59 ; \mathrm{N}, 12.91$; Found: $\mathrm{C}, 55.72 ; \mathrm{H}$, $9.91 ; \mathrm{N}, 12.83$. 
Reduction of the azide $(3.70 \mathrm{~g}, 11.4 \mathrm{mmol})$ in THF $(18 \mathrm{~mL})$ with LAH (1M in THF, 17.1 $\mathrm{mL}, 0.64 \mathrm{~g}, 17.1 \mathrm{mmol}$ ) followed by purification by column chromatography on silica gel (pet-ether:EtOAc $=90: 10)$ provided the amine $(\mathbf{\pm})-3 \mathbf{b}$ as a pure oil $(3.20 \mathrm{~g}$, yield $95 \%$ ). $R_{f} 0.36$ (pet-ether:EtOAc = 8:2). IR (neat film) 3380, 2960, 2930, 2898, 2857, 2163, 1591, 1472, 1377, 1251, 1139, 1086, $1001 \mathrm{~cm}^{-1} ;{ }^{1} \mathrm{H}$ NMR $\left(600 \mathrm{MHz}, \mathrm{CDCl}_{3}\right) \delta 4.08$ (qdd, $J=6,6,5.4 \mathrm{~Hz}, 1 \mathrm{H}$ ), $3.63(\mathrm{dd}, J=7.8,7.2 \mathrm{~Hz}, 1 \mathrm{H}$ ), 1.77 (ddd, $J=13.2,7.2,6 \mathrm{~Hz}$, 1H), 1.62 (ddd, $J=13.2,7.8,5.4 \mathrm{~Hz}, 1 \mathrm{H}$ ), 1.44 (brs, 2H), 1.15 (d, $J=6 \mathrm{~Hz}, 3 \mathrm{H}$ ), 0.89 (s, 9H), 0.15 (s, 9H), 0.074 (d, J = 2.4 Hz, 6H); $\left.{ }^{13} \mathrm{C} \mathrm{NMR} \mathrm{(150} \mathrm{MHz,} \mathrm{CDCl}_{3}\right) \delta 110.48$, 86.50, 65.98, 47.55, 41.21, 26.12, 23.94, 18.27, 0.24, -4.13, -4.49; HRMS (FAB ${ }^{+}$) Calcd. for $\mathrm{C}_{15} \mathrm{H}_{34} \mathrm{ONSi}_{2}\left[(\mathrm{M}+\mathrm{H})^{+}\right]$300.2179, found 307.2177. Anal. Calcd. for $\mathrm{C}_{15} \mathrm{H}_{33} \mathrm{ONSi}_{2}$ : C, 60.13; H, 11.10; N, 4.68; Found: C, 60.27; H, 11.23; N, 4.77.

\section{General procedure for preparation of alkynyl alcohol substrates $4-6$ :}

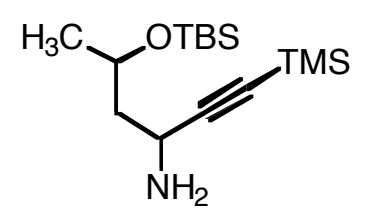

$( \pm)-3 a$ or $3 b$

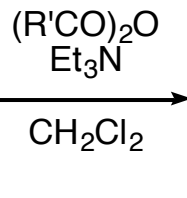

$\mathrm{R}^{\prime}=$ Boc, Acetyl, or Pivaloyl

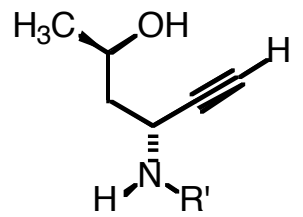

diastereomer a

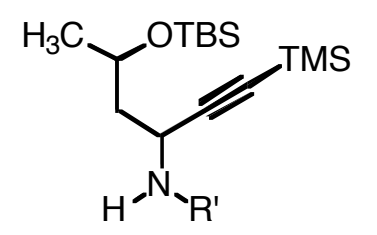

$\underset{{ }^{\circ} \mathrm{C}}{\stackrel{\substack{\text { TBAF } \\ \text { THF }}}{\longrightarrow}}$

or<smiles>C#CC(C[C@@H](C)O)NP</smiles>

diastereomer $b$
4: $\mathrm{R}^{\prime}=\mathrm{Boc}$

5: $\mathrm{R}^{\prime}=$ Acetyl

6: $R^{\prime}=$ Pivaloyl

$\mathrm{N}$-acylation: To a stirred solution of $3 \mathbf{a}$ or $3 \mathbf{b}(0.300 \mathrm{~g}, 1 \mathrm{mmol})$ in $\mathrm{CH}_{2} \mathrm{Cl}_{2}(3 \mathrm{~mL})$ at $0{ }^{\circ} \mathrm{C}$ was added $\mathrm{Et}_{3} \mathrm{~N}(0.303 \mathrm{~g}, 0.41 \mathrm{~mL}, 3 \mathrm{mmol})$ and DMAP $(0.012 \mathrm{~g}, 0.10 \mathrm{mmol})$. Anhydride $(3 \mathrm{mmol})$ in $\mathrm{CH}_{2} \mathrm{Cl}_{2}(2 \mathrm{~mL})$ was added dropwise. The reaction was stirred at $0{ }^{\circ} \mathrm{C}$ for 4 hours and then allowed to come to room temperature. If starting material remains as determined by TLC, additional anhydride is added. The reaction was quenched by evaporation of solvent, taken up in EtOAc $(20 \mathrm{~mL})$, washed with $\mathrm{NaHCO}_{3}$ $\left(20 \mathrm{~mL}\right.$ ), dried over $\mathrm{Na}_{2} \mathrm{SO}_{4}$, and evaporated under reduced pressure. Purification of the material by flash column chromatography on silica gel afforded the $\mathrm{N}$-acylated compounds. 
Desilylation: Removal of the O-TBS and alkynyl-TMS groups was accomplished on 1 mmol scale by dissolving in THF $(1 \mathrm{~mL})$ at $0{ }^{\circ} \mathrm{C}$ and adding TBAF (1M in THF, $4 \mathrm{~mL}, 4$ $\mathrm{mmol})$. The solution was stirred for $4 \mathrm{~h}$ at $0{ }^{\circ} \mathrm{C}$, then brought to room temperature. If starting material remains as determined by TLC, additional TBAF is added. The reaction was quenched with $\mathrm{H}_{2} \mathrm{O}(20 \mathrm{~mL})$. The organic phase was separated and the aqueous layer was extracted with EtOAc $(3 \times 20 \mathrm{~mL})$. The organic layers were combined, washed with a saturated solution of $\mathrm{NaHCO}_{3}(20 \mathrm{~mL})$, dried over $\mathrm{Na}_{2} \mathrm{SO}_{4}$, and evaporated under reduced pressure. The residual oil was purified by column chromatography on silica gel to yield alkynyl alcohol substrates (4a-6a) or (4b-6b).

$\left(3 R^{*}, 5 R^{*}\right)$-3-amino-hex-1-yn-5-ol, $N$-(tert-Butoxycarbonyl) derivative ( \pm )-4a: The general procedure for $\mathrm{N}$-acylation was followed starting with $3 \mathrm{a}(1.2 \mathrm{~g}, 4 \mathrm{mmol}), \mathrm{Et}_{3} \mathrm{~N}$ (0.56 mL, $4 \mathrm{mmol})$ in $\mathrm{CH}_{2} \mathrm{Cl}_{2}(20 \mathrm{~mL})$ and di-tert-butyl dicarbonate $(0.873 \mathrm{~g}, 4 \mathrm{mmol})$ without DMAP. Purification by column chromatography on silica gel (pet-ether:EtOAc $=$ $90: 10)$ provided the $\mathrm{N}$-Boc derivative of $3 \mathrm{a}$ as a white solid (1.48 g, yield $93 \%$ ). Mp 60.5-62.5 ${ }^{\circ} \mathrm{C}$; IR (neat film) 3328, 3113, 2965, 2930, 2902, 2857, 2180, 1679, 1526 , 1462, 1391, 1366, 1329, 1249, 1167, 1090, $1024 \mathrm{~cm}^{-1} ;{ }^{1} \mathrm{H} \mathrm{NMR}\left(600 \mathrm{MHz}, \mathrm{CDCl}_{3}\right) \delta$ 4.75 (brs, 1H), 4.51 (brs, 1H), $4.01(\mathrm{~m}, 1 \mathrm{H}), 1.90-1.51(\mathrm{~m}, 2 \mathrm{H}), 1.45(\mathrm{~s}, 9 \mathrm{H}), 1.18(\mathrm{~d}, J=$ $9.6 \mathrm{~Hz}, 3 \mathrm{H}), 0.90(\mathrm{~s}, 9 \mathrm{H}), 0.16(\mathrm{~s}, 9 \mathrm{H}), 0.088(\mathrm{~d}, J=17.4 \mathrm{~Hz}, 6 \mathrm{H}) ;{ }^{13} \mathrm{C}$ NMR $(150 \mathrm{MHz}$, $\left.\mathrm{CDCl}_{3}\right) \delta 154.74,105.73,87.90,79.79,66.15,46.06,41.72,28.58,26.13,24.37,18.24$, 0.13, -1.06, -4.05; HRMS (FAB ${ }^{+}$) Calcd. for $\mathrm{C}_{20} \mathrm{H}_{41} \mathrm{O}_{3} \mathrm{NSi}_{2} \mathrm{Li}\left[(\mathrm{M}+\mathrm{Li})^{+}\right]$406.2785, found 406.2796. Anal. Calcd. for $\mathrm{C}_{20} \mathrm{H}_{42} \mathrm{O}_{3} \mathrm{NSi}_{2}$ : C, 59.95; $\mathrm{H}, 10.56 ; \mathrm{N}, 3.50$; Found $\mathrm{C}, 60.07$; $\mathrm{H}, 10.39$; N, 3.50 .

The general procedure for desilylation was followed starting with the above-described $N$-Boc derivative of $3 \mathbf{a}(0.800 \mathrm{~g}, 2 \mathrm{mmol})$ in THF $(8 \mathrm{~mL})$ to which was added acetic acid $(0.12 \mathrm{~mL}, 2 \mathrm{mmol})$ and then TBAF $(1 \mathrm{M}$ in THF, $12 \mathrm{~mL}, 12 \mathrm{mmol})$. Purification by column chromatography on silica gel (pet-ether:EtOAc $=7: 3$ ) provided $4 \mathrm{a}$ as an oil (0.400 g, yield 95\%). IR (neat film) 3308, 3312, 2975, 2963, 2932, 2121, 1692, 1519, 1368, 1278, 1250, 1170, 1083, $1047 \mathrm{~cm}^{-1} ;{ }^{1} \mathrm{H}$ NMR (400 MHz, $\left.\mathrm{CDCl}_{3}\right) \delta 4.83$ (brs, $J=$ $1 \mathrm{H}), 4.66-4.56(\mathrm{~m}, 1 \mathrm{H}), 4.10-3.98(\mathrm{~m}, 1 \mathrm{H}), 2.33(\mathrm{~d}, J=2 \mathrm{~Hz}, 1 \mathrm{H}), 1.92-1.78(\mathrm{~m}, 2 \mathrm{H})$, $1.46(\mathrm{~s}, 9 \mathrm{H}), 1.26(\mathrm{~d}, J=6 \mathrm{~Hz}, 3 \mathrm{H}) ;{ }^{13} \mathrm{C}$ NMR $\left(150 \mathrm{MHz}, \mathrm{CDCl}_{3}\right) \delta$ 155.11, 83.54, 80.40, 71.77, 65.68, 45.30, 41.01, 28.54, 24.05, HRMS (FAB ${ }^{+}$) Calcd. for $\mathrm{C}_{11} \mathrm{H}_{19} \mathrm{O}_{3} \mathrm{NLi}[(\mathrm{M}+$ $\left.\mathrm{Li}^{+}\right]$220.1525, found 220.1534. Anal. Calcd. for $\mathrm{C}_{11} \mathrm{H}_{19} \mathrm{O}_{3} \mathrm{~N}$ : C, 61.95; $\mathrm{H}, 9.00 ; \mathrm{N}, 6.57$; Found: $\mathrm{C}, 61.49 ; \mathrm{H}, 9.06 ; \mathrm{N}, 6.41$. 
$\left(3 S^{*}, 5 R^{*}\right)$-3-amino-hex-1-yn-5-ol, $N$-(tert-Butoxycarbonyl) derivative ( \pm )-4b: The general procedure for acylation was followed starting with $\mathbf{3 b}$ with modifications as for 3a. Purification by column chromatography on silica gel (pet-ether:EtOAc $=90: 10$ ) provided the $\mathrm{N}$-Boc derivative of $3 \mathbf{b}$ as a white solid (yield $93 \%$ ). Mp $98.5-100.5{ }^{\circ} \mathrm{C}$; IR (neat film) 3331, 3128, 2965, 2930, 2898, 2858, 2171, 1683, 1523, 1472, 1393, 1369, 1279, 1250, 1176, 1092, $1016 \mathrm{~cm}^{-1} ;{ }^{1} \mathrm{H}$ NMR $\left(600 \mathrm{MHz}, \mathrm{CDCl}_{3}\right) \delta 5.56(\mathrm{~d}, J=5.4 \mathrm{~Hz}$, $1 \mathrm{H}$ ); 4.49 (brs, $1 \mathrm{H}), 4.26$ (brs, $1 \mathrm{H}), 1.80-1.70(\mathrm{~m}, 2 \mathrm{H}), 1.44$ (s, 9H), 1.18 (d, J = 6.6 Hz, $3 \mathrm{H}), 0.92(\mathrm{~s}, 9 \mathrm{H}), 0.15(\mathrm{~s}, 9 \mathrm{H}), 0.12(\mathrm{~d}, J=19.2 \mathrm{~Hz}, 6 \mathrm{H}) ;{ }^{13} \mathrm{C} \mathrm{NMR}\left(150 \mathrm{MHz}, \mathrm{CDCl}_{3}\right) \delta$ 155.06, 105.46, 87.37, 79.53, 66.94, 43.72, 41.91, 28.56, 26.13, 24.18, 18.19, 0.16, -3.86, -4.49; HRMS (FAB $\left.{ }^{+}\right)$Calcd. for $\mathrm{C}_{20} \mathrm{H}_{41} \mathrm{O}_{3} \mathrm{NSi}_{2} \mathrm{Li}\left[(\mathrm{M}+\mathrm{Li})^{+}\right]$406.2785, found 406.2781. Anal. Calcd. for $\mathrm{C}_{20} \mathrm{H}_{42} \mathrm{O}_{3} \mathrm{NSi}_{2}$ : C, 59.95; $\mathrm{H}, 10.56 ; \mathrm{N}, 3.50$; Found $\mathrm{C}, 60.34$; $\mathrm{H}, 10.58$; N, 3.40 .

The general procedure for desilylation was followed starting with the $\mathrm{N}$-Boc derivative of $\mathbf{3 b}$ with modifications as for $\mathbf{4 a}$. Purification by column chromatography on silica gel (pet-ether:EtOAc $=7: 3$ ) provided $\mathbf{4 b}$ as a white solid (yield 95\%). Mp 91-92 ${ }^{\circ} \mathrm{C} ; \quad I R$ (neat film) 3404, 3312, 3245, 3127, 3010, 2980, 2963, 2911, 2121, 1673, 1534, 1397 , 1276, 1258, 1164, $1077 \mathrm{~cm}^{-1} ;{ }^{1} \mathrm{H}$ NMR $\left(600 \mathrm{MHz}, \mathrm{CDCl}_{3}\right) \delta 5.15(\mathrm{~d}, J=8.4 \mathrm{~Hz}, 1 \mathrm{H})$, 4.40 (brs, 1H), 3.91 (brs, 1H), 3.42 (brs, 1H), 2.32-2.29 (m, 1H), 1.83-1.68 (m, 2H), 1.45 $(\mathrm{s}, 9 \mathrm{H}), 1.21(\mathrm{~d}, J=6.6 \mathrm{~Hz}, 3 \mathrm{H}) ;{ }^{13} \mathrm{C}$ NMR $\left(150 \mathrm{MHz}, \mathrm{CDCl}_{3}\right) \delta$ 156.27, 83.27, 80.70, 71.56, 63.98, 46.03, 40.38, 28.50, 23.02, HRMS (FAB ${ }^{+}$) Calcd. for $\mathrm{C}_{11} \mathrm{H}_{19} \mathrm{O}_{3} \mathrm{NLi}[(\mathrm{M}+$ $\left.\mathrm{Li}^{+}{ }^{+}\right]$220.1525, found 220.1533. Anal. Calcd. for $\mathrm{C}_{11} \mathrm{H}_{19} \mathrm{O}_{3} \mathrm{~N}: \mathrm{C}, 61.95 ; \mathrm{H}, 9.00 ; \mathrm{N}, 6.57$; Found: $\mathrm{C}, 61.86 ; \mathrm{H}, 9.10 ; \mathrm{N}, 6.45$.

$\left(3 R^{\star}, 5 R^{\star}\right)$-3-amino-hex-1-yn-5-ol, $N$-acetyl derivative $( \pm)$-5a: The general procedure for acylation was followed starting with $3 a$ and acetic anhydride. Purification by column chromatography on silica gel (pet-ether:EtOAc $=80: 20$ ) provided the $\mathrm{N}$-Ac derivative as a white solid (yield 96\%). Mp 80.5-82.5 ${ }^{\circ} \mathrm{C}$; IR (neat film) 3421, 3248, 3066, 2960, 2930, 2896, 2857, 2173, 1645, 1563, 1473, 1376, 1251, 1147, 1067, $1017 \mathrm{~cm}^{-1} ;{ }^{1} \mathrm{H}$ NMR $\left(600 \mathrm{MHz}, \mathrm{CDCl}_{3}\right) \delta 5.66(\mathrm{~d}, J=6.3 \mathrm{~Hz}, 1 \mathrm{H}), 4.92$ (ddd, $J=9.3,9,6.3 \mathrm{~Hz}, 1 \mathrm{H}$ ), 4.01 (dqd, $J=9.6,6.6,4.2 \mathrm{~Hz}, 1 \mathrm{H}$ ), 1.98 (s, 3H), 1.83 (ddd, $J=13.8,9.3,4.2 \mathrm{~Hz}, 1 \mathrm{H}$ ), 163-157 (m, 1H), 1.19 (d, J = 6.6 Hz, 3H), $0.91(\mathrm{~s}, 9 \mathrm{H}), 0.17(\mathrm{~s}, 9 \mathrm{H}), 0.092(\mathrm{~d}, J=18$ 
$\mathrm{Hz}, 6 \mathrm{H}) ;{ }^{13} \mathrm{C}$ NMR $\left(150 \mathrm{MHz}, \mathrm{CDCl}_{3}\right) \delta$ 168.58, 105.40, 88.14, 66.10, 45.91, 40.23, 26.09, 24.33, 23.56, 18.23, 0.12, -3.97, -4.64; HRMS (FAB ${ }^{+}$) Calcd. for $\mathrm{C}_{17} \mathrm{H}_{35} \mathrm{O}_{2} \mathrm{NSi}_{2} \mathrm{Li}$ $\left[(\mathrm{M}+\mathrm{Li})^{+}\right]$348.2366, found 348.2360. Anal. Calcd. for $\mathrm{C}_{17} \mathrm{H}_{35} \mathrm{O}_{2} \mathrm{NSi}_{2}: \mathrm{C}, 59.77 ; \mathrm{H}$, 10.33; N, 4.10; Found C, 59.81; H, 10.38; N, 4.11.

The general procedure for desilylation was followed, and purification by column chromatography on silica gel $\left(\mathrm{CH}_{2} \mathrm{Cl}_{2}: \mathrm{CH}_{3} \mathrm{OH}=97: 3\right)$ provided $5 \mathrm{a}$ as an oil (yield $92 \%$ ). IR (neat film) 3284, 3069, 2967, 2918, 2849, 2115, 1650, 1541, 1461, 1434, 1374, 1293 $\mathrm{cm}^{-1} ;{ }^{1} \mathrm{H}$ NMR $\left(600 \mathrm{MHz}, \mathrm{CDCl}_{3}\right) \delta 6.03$ (brs, $1 \mathrm{H}$ ), 4.90 (ddd, $J=14.4,7.8,2.4 \mathrm{~Hz}, 1 \mathrm{H}$ ), 4.02 (brs, 1H), 2.33 (d, J = 2.4 Hz, 1H), $2.0(\mathrm{~s}, 3 \mathrm{H}), 1.88-1.75(\mathrm{~m}, 2 \mathrm{H}), 1.25$ (d, J = 6.6 $\mathrm{Hz}, 3 \mathrm{H}) ;{ }^{13} \mathrm{C}$ NMR $\left(150 \mathrm{MHz}, \mathrm{CDCl}_{3}\right) \delta 169.58,83.27,71.84,65.74,44.92,39.76,24.11$, 23.45; HRMS (EI) Calcd. for $\mathrm{C}_{8} \mathrm{H}_{13} \mathrm{O}_{2} \mathrm{NLi}\left[(\mathrm{M})^{+}\right]$155.0946, found 155.0953.

$\left(3 S^{*}, 5 R^{*}\right)$-3-amino-hex-1-yn-5-ol, $N$-acetyl derivative $( \pm)-5 \mathrm{~b}$ : The general procedure for acylation was followed starting with $\mathbf{3 b}$. Purification by column chromatography on silica gel (pet-ether:EtOAc $=80: 20$ ) provided the $\mathrm{N}$-Ac derivative as a white solid (yield 80\%). Mp 79-80 ${ }^{\circ} \mathrm{C}$; IR (neat film) 3438, 3295, 3125, 2958, 2931, 2896, 2856, 2176, 1656, 1539, 1400, 1255, 1123, 1081, $1013 \mathrm{~cm}^{-1} ;{ }^{1} \mathrm{H}$ NMR $\left(600 \mathrm{MHz}, \mathrm{CDCl}_{3}\right) \delta 6.60(\mathrm{~d}, J$ $=7.8 \mathrm{~Hz}, 1 \mathrm{H}), 4.90-4.86(\mathrm{~m}, 1 \mathrm{H}), 4.35-4.30(\mathrm{~m}, 1 \mathrm{H}), 1.95(\mathrm{~s}, 3 \mathrm{H}), 1.77$ (ddd, $J=13.8$, 10.2, $5.4 \mathrm{~Hz}, 1 \mathrm{H}$ ), 1.72 (ddd, $J=13.8,9,4.8 \mathrm{~Hz}, 1 \mathrm{H}), 1.20$ (d, $J=6 \mathrm{~Hz}, 3 \mathrm{H}$ ), 0.94 (s, $9 \mathrm{H}), 0.16(\mathrm{~s}, 9 \mathrm{H}), 0.13(\mathrm{~s}, 6 \mathrm{H}) ;{ }^{13} \mathrm{C}$ NMR $\left(150 \mathrm{MHz}, \mathrm{CDCl}_{3}\right) \delta 168.66,104.64,87.59$, $67.34,43.25,40.37,26.16,24.22,23.54,18.22,0.16,-4.01,-4.15$; HRMS (FAB $\left.{ }^{+}\right)$ Calcd. for $\mathrm{C}_{17} \mathrm{H}_{35} \mathrm{O}_{2} \mathrm{NSi}_{2} \mathrm{Li}\left[(\mathrm{M}+\mathrm{Li})^{+}\right]$348.2366, found 348.2358. Anal. Calcd. for $\mathrm{C}_{17} \mathrm{H}_{35} \mathrm{O}_{2} \mathrm{NSi}_{2}$ : C, 59.77; $\mathrm{H}, 10.33 ; \mathrm{N}, 4.10$; Found $\mathrm{C}, 59.92 ; \mathrm{H}, 10.43 ; \mathrm{N}, 4.15$.

The general procedure for desilylation was followed, and purification by column chromatography on silica gel $\left(\mathrm{CH}_{2} \mathrm{Cl}_{2}: \mathrm{CH}_{3} \mathrm{OH}=97: 3\right)$ provided $\mathbf{5 b}$ as an oil (yield $\left.80 \%\right)$. IR (neat film) 3362, 3280, 3057, 2961, 2919, 2850, 2354, 1658, 1540, 1463, 1375, 1287, $1070 \mathrm{~cm}^{-1} ;{ }^{1} \mathrm{H}$ NMR $\left(600 \mathrm{MHz}, \mathrm{CDCl}_{3}\right) \delta 6.13$ (brs, $\left.1 \mathrm{H}\right), 4.94-4.90(\mathrm{~m}, 1 \mathrm{H}), 3.95-$ $3.85(\mathrm{~m}, 1 \mathrm{H}), 3.38(\mathrm{~d}, J=4.2 \mathrm{~Hz}, 1 \mathrm{H}), 2.29(\mathrm{~d}, J=2.4 \mathrm{~Hz}, 1 \mathrm{H}), 2.01(\mathrm{~s}, 3 \mathrm{H}), 1.76-1.56$ $(\mathrm{m}, 1 \mathrm{H}), 1.45-1.35(\mathrm{~m}, 1 \mathrm{H}), 1.19(\mathrm{~d}, J=6 \mathrm{~Hz}, 3 \mathrm{H}) ;{ }^{13} \mathrm{C} \mathrm{NMR}\left(150 \mathrm{MHz}, \mathrm{CDCl}_{3}\right) \delta$ $170.55,82.87,71.80,64.08,45.35,39.43,23.09,20.17$; HRMS (EI) Calcd. for $\mathrm{C}_{8} \mathrm{H}_{13} \mathrm{O}_{2} \mathrm{~N}\left[(\mathrm{M})^{+}\right]$155.09463, found 155.0953. 
$\left(3 R^{*}, \quad 5 R^{\star}\right)$-3-amino-hex-1-yn-5-ol, $N$-pivaloyl derivative $( \pm)-6 \mathrm{a}$ : The general procedure for acylation was followed starting with $\mathbf{3 b}$. Purification by column chromatography on silica gel (pet-ether:EtOAc $=80: 20$ ) provided the $N$-Piv derivative as a white solid (yield $88 \%$ ). Mp 114-116 ${ }^{\circ} \mathrm{C}$; IR (neat film) 3315, 3126, 2960, 2931, 2901 , $2859,2174,1638,1530,1473,1400,1251,1151,1009, \mathrm{~cm}^{-1} ;{ }^{1} \mathrm{H}$ NMR $(600 \mathrm{MHz}$, $\left.\mathrm{CDCl}_{3}\right) \delta 5.70(\mathrm{~d}, J=8.4 \mathrm{~Hz}, 1 \mathrm{H}$ ), 4.91 (ddd, $J=9.3,8.4,5.4 \mathrm{~Hz}, 1 \mathrm{H}), 3.99$ (dq, $J=9.6$, $6 \mathrm{~Hz}, 1 \mathrm{H}$ ), 1.82 (ddd, $J=13.2,9,5.4 \mathrm{~Hz}, 1 \mathrm{H}$ ), 1.55 (ddd, $J=13.2,9.6,3.6 \mathrm{~Hz}, 1 \mathrm{H}$ ), 1.20 (s, 9H), $1.18(\mathrm{~d}, J=6 \mathrm{~Hz}, 3 \mathrm{H}), 0.95(\mathrm{~s}, 9 \mathrm{H}), 0.17(\mathrm{~s}, 9 \mathrm{H}), 0.087(\mathrm{~d}, J=22 \mathrm{~Hz}, 6 \mathrm{H}) ;{ }^{13} \mathrm{C}$ NMR $\left(150 \mathrm{MHz}, \mathrm{CDCl}_{3}\right) \delta 176.98,105.65,88.15,65.95,46.10,40.06,38.77,27.68$, 26.12, 24.31, 18.27, 0.12, -4.05, -4.60; HRMS (FAB ${ }^{+}$Calcd. for $\mathrm{C}_{20} \mathrm{H}_{42} \mathrm{O}_{2} \mathrm{NSi}_{2} \mathrm{Li}[(\mathrm{M}+$ $\left.\mathrm{H})^{+}\right]$384.2754, found 384.2752. Anal. Calcd. for $\mathrm{C}_{20} \mathrm{H}_{41} \mathrm{O}_{2} \mathrm{NSi}_{2}$ : C, 62.60; $\mathrm{H}, 10.77 ; \mathrm{N}$, 3.65; Found C, 62.55; H, 11.00; N, 3.60.

The general procedure for desilylation was followed, and purification by column chromatography on silica gel (pet-ether:EtOAc $=8: 2$ ) provided $6 \mathbf{a}$ as a white solid (yield 87\%). Mp 61-63 ${ }^{\circ} \mathrm{C}$; IR (neat film) 3353, 3312, 2967, 2932, 2873, 2116, 1643, 1527, 1482, 1461, 1368, 1206, $1137 \mathrm{~cm}^{-1} ;{ }^{1} \mathrm{H}$ NMR $\left(600 \mathrm{MHz}, \mathrm{CDCl}_{3}\right) \delta 6.04(\mathrm{~d}, J=5.4 \mathrm{~Hz}$, 1H), 4.87 (ddd, $J=7.2,7.2,4.2 \mathrm{~Hz}, 1 \mathrm{H}), 4.01$ (qd, $J=6.6,6 \mathrm{~Hz}, 1 \mathrm{H}), 2.33(\mathrm{~d}, J=2.4$ $\mathrm{Hz}, 1 \mathrm{H}$ ), 2.23 (brs, 1H), 1.84 (ddd, $J=7.2,7.2,4.2 \mathrm{~Hz}, 2 \mathrm{H}) 1.25$ (d, J = 6.6 Hz, 3H), 1.20 (s, 9H); ${ }^{13} \mathrm{C}$ NMR $\left(150 \mathrm{MHz}, \mathrm{CDCl}_{3}\right) \delta 177.97,83.56,71.77,65.96,45.06,39.88$, 38.80, 27.59, 24.13; HRMS (FAB ${ }^{+}$) Calcd. for $\mathrm{C}_{11} \mathrm{H}_{19} \mathrm{O}_{2} \mathrm{~N} \mathrm{Li}\left[(\mathrm{MLi})^{+}\right]$204.1576, found 204.1576 .

$\left(3 S^{*}, \quad 5 R^{*}\right)$-3-amino-hex-1-yn-5-ol, $N$-pivaloyl derivative $( \pm)-6 \mathrm{~b}$ : The general procedure for acylation was followed starting with $\mathbf{3 b}$. Purification by column chromatography on silica gel (pet-ether:EtOAc $=95: 5$ ) provided the $N$-Piv derivative as a white solid (yield 81\%). Mp 66-68 ' C; IR (neat film) 3438, 3337, 2958, 2930, 2901, $2858,2171,1642,1523,1473,1367,1251,1121,1006, \mathrm{~cm}^{-1} ;{ }^{1} \mathrm{H}$ NMR $(600 \mathrm{MHz}$, $\left.\mathrm{CDCl}_{3}\right) \delta 6.27(\mathrm{~d}, J=8.4 \mathrm{~Hz}, 1 \mathrm{H}), 4.90-4.86(\mathrm{~m}, 1 \mathrm{H}), 4.19-4.14(\mathrm{~m}, 1 \mathrm{H}), 1.79-1.70(\mathrm{~m}$, $1 \mathrm{H}), 1.72-1.64(\mathrm{~m}, 1 \mathrm{H}), 1.16(\mathrm{brs}, 12 \mathrm{H}), 0.89(\mathrm{~s}, 9 \mathrm{H}), 0.13(\mathrm{~s}, 9 \mathrm{H}), 0.11(\mathrm{~d}, J=6 \mathrm{H}) ;{ }^{13} \mathrm{C}$ NMR $\left(150 \mathrm{MHz}, \mathrm{CDCl}_{3}\right) \delta 177.14,105.17,87.55,66.98,44.27,40.04,38.65,27.66$, 26.23, 24.04, 18.41, 0.16, -3.62, -4.70; HRMS (FAB $)$ Calcd. for $\mathrm{C}_{20} \mathrm{H}_{41} \mathrm{O}_{2} \mathrm{NSi}_{2} \mathrm{Li}[(\mathrm{M}+$ 
$\left.\mathrm{Li}^{+}\right]$390.2836, found 390.2835. Anal. Calcd. for $\mathrm{C}_{20} \mathrm{H}_{41} \mathrm{O}_{2} \mathrm{NSi}_{2}$ : C, 62.60; $\mathrm{H}, 10.77 ; \mathrm{N}$, 3.65; Found C, 62.79; H, 10.87; N, 3.61.

The general procedure for desilylation was followed, and purification by column chromatography on silica gel (pet-ether:EtOAc $=8: 2$ ) provided $\mathbf{6 b}$ as a white solid (yield 87\%). Mp 93-95 ' C; IR (neat film) 3383, 3276, 3214, 3248, 2973, 2945, 2912, 2116 , 1620, 1533, 1401, 1331, 1204, $1140 \mathrm{~cm}^{-1} ;{ }^{1} \mathrm{H}$ NMR $\left(600 \mathrm{MHz}, \mathrm{CDCl}_{3}\right) \delta 6.30$ (d, $J=4.8$ $\mathrm{Hz}, 1 \mathrm{H}), 4.94-4.91(\mathrm{~m}, 1 \mathrm{H}), 3.86-3.79(\mathrm{~m}, 1 \mathrm{H}), 3.65(\mathrm{~d}, J=1.8 \mathrm{~Hz}, 1 \mathrm{H}), 2.32$ (ddd, $J=$ 1.8, 1.8, 1.8 Hz, 1H), 1.79 (ddd, $J=13.2,10.2,3 \mathrm{~Hz}, 1 \mathrm{H}$ ), 1.72 (ddd, $J=13.2,10.8,1.2$ $\mathrm{Hz}, 1 \mathrm{H}), 1.22(\mathrm{~d}, J=0.6 \mathrm{~Hz}, 9 \mathrm{H}), 1.21(\mathrm{~d}, J=6.3 \mathrm{~Hz}, 3 \mathrm{H}) ;{ }^{13} \mathrm{C} \mathrm{NMR}\left(150 \mathrm{MHz}, \mathrm{CDCl}_{3}\right) \delta$ 179.21, 83.16, 71.73, 63.96, 45.57, 39.31, 38.91, 27.67, 23.06; HRMS (EI) Calcd. for $\mathrm{C}_{11} \mathrm{H}_{19} \mathrm{O}_{2} \mathrm{~N}\left[(\mathrm{M})^{+}\right]$197.14158, found 197.14244.

$\left(3 R^{\star}, 5 R^{\star}\right)$-3-amino-hex-1-yn-5-ol, $N$-formyl derivative $( \pm)-7 \mathrm{a}$ :

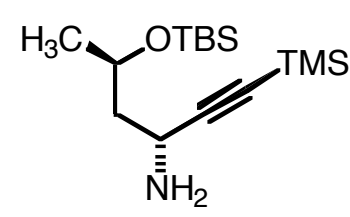

$( \pm)-3 a$

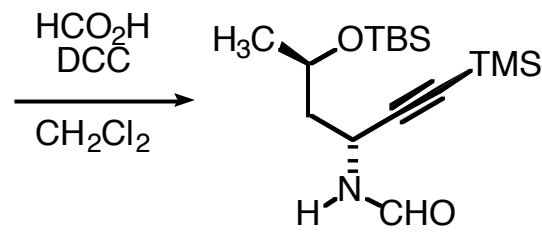

$\mathrm{H}^{-\overline{\mathrm{N}}_{\mathrm{CHO}}}$

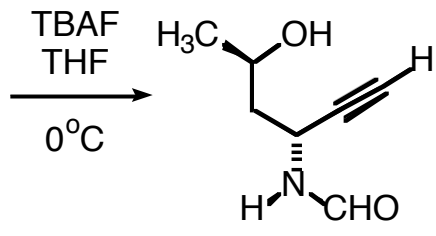

$( \pm)-7 \mathrm{a}$

To a stirred solution of $3 \mathrm{a}(2.5 \mathrm{~g}, 8 \mathrm{mmol})$ in $\mathrm{CH}_{2} \mathrm{Cl}_{2}(16 \mathrm{~mL})$ was added a solution of formic acid $(0.736 \mathrm{~g}, 0.6 \mathrm{~mL}, 16 \mathrm{mmol})$ in $\mathrm{CH}_{2} \mathrm{Cl}_{2}(32 \mathrm{~mL})$ at room temperature, after which 1,3-dicyclohexylcarbodiimide (DCC, $3.3 \mathrm{~g}, 16 \mathrm{mmol}$ ) was added neat and the reaction progress followed by TLC. The reaction was stirred for $6 \mathrm{~h}$ at which time the reaction was quenched by addition of a solution of $5 \% \mathrm{NaOH},(60 \mathrm{~mL})$. The organic phase was separated and the aqueous layer was extracted with $\mathrm{CH}_{2} \mathrm{Cl}_{2}(3 \times 25 \mathrm{~mL})$. The combined organic layers were washed with a solution of $1 \% \mathrm{HCl}(60 \mathrm{~mL})$, dried over $\mathrm{Na}_{2} \mathrm{SO}_{4}$, and evaporated under reduced pressure. The residual oil was purified by column chromatography on silica gel (pet-ether:EtOAc $=8: 2$ ) to yield the $N$-formyl derivative of $3 \mathbf{a}$ as a white solid $\left(1.84 \mathrm{~g}\right.$, yield $70 \%$ ). Mp $110-111^{\circ} \mathrm{C}$; IR (neat film) 3261, 3058, 2958, 2928, 2890, 2855, 2178, 1684, 1657, 1546, 1473, 1382, 1249, 1146 , 1068, $1004 \mathrm{~cm}^{-1} ;{ }^{1} \mathrm{H}$ NMR (600 MHz, $\left.\mathrm{CDCl}_{3}\right) \delta 8.20(\mathrm{~d}, J=12 \mathrm{~Hz}, 0.3 \mathrm{H}), 8.12(\mathrm{~s}, 0.7 \mathrm{H})$, 
5.8-5.65 (m, 1H), 4.97 (ddd, $J=9.6,9,5.4 \mathrm{~Hz}, 0.7 \mathrm{H}), 4.38$ (ddd, $J=9.6,9,5.4 \mathrm{~Hz}$, $0.3 \mathrm{H}$ ), 4.03 (dqd, $J=9.6,6.6,3.6 \mathrm{~Hz}, 1 \mathrm{H}), 1.86$, (ddd, $J=13.8,9,5.4 \mathrm{~Hz}, 1 \mathrm{H}$ ), 1.64 (ddd, $J=13.8,9.6,2.4 \mathrm{~Hz}, 1 \mathrm{H}), 1.20(\mathrm{~d}, J=6.6 \mathrm{~Hz}, 3 \mathrm{H}), 0.911(\mathrm{~s}, 9 \mathrm{H}), 0.17(\mathrm{~s}, 9 \mathrm{H})$, $0.10(\mathrm{~d}, J=16.2 \mathrm{~Hz}, 6 \mathrm{H}) ;{ }^{13} \mathrm{C}$ NMR $\left(150 \mathrm{MHz}, \mathrm{CDCl}_{3}\right) \delta 159.68,104.59,88.55,66.12$, 45.59, 39.02, 26.09, 24.32, 18.22, 0.062, -3.95, -4.63; HRMS (FAB ${ }^{+}$) Calcd. for $\mathrm{C}_{16} \mathrm{H}_{33} \mathrm{O}_{2} \mathrm{NSi}_{2} \mathrm{Li}\left[(\mathrm{M}+\mathrm{Li})^{+}\right]$334.2210, found 334.2197. Anal. Calcd. for $\mathrm{C}_{16} \mathrm{H}_{33} \mathrm{O}_{2} \mathrm{NSi}_{2}$ : C, 58.66; H, 10.15; N, 4.28; Found: C, 58.99; H, 10.20; N, 4.19.

The general procedure for desilylation was followed, and purification by column chromatography on silica gel $\left(\mathrm{CH}_{2} \mathrm{Cl}_{2}: \mathrm{CH}_{3} \mathrm{OH}=98: 2\right)$ provided $7 \mathrm{a}$ as an oil (yield $87 \%$ ). IR (neat film) 3286, 3040, 2969, 2926, 2878, 2764, 2118, 1667, 1536, 1461, 1384 1270, 1137, $1086 \mathrm{~cm}^{-1} ;{ }^{1} \mathrm{H}$ NMR $\left(600 \mathrm{MHz}, \mathrm{CDCl}_{3}\right) \delta 8.26(\mathrm{~d}, J=12 \mathrm{~Hz}, 0.2 \mathrm{H}), 8.15(\mathrm{~s}, 0.8 \mathrm{H})$, 6.14 (brs, $1 \mathrm{H}$ ), 4.99 (ddd, $J=8.2,8.2,1.8 \mathrm{~Hz}, 0.8 \mathrm{H}$ ), 4.51 (ddd, $J=8.2,8.2,1.8 \mathrm{~Hz}$, $0.2 \mathrm{H}$ ), 4.05 (brs, $1 \mathrm{H}), 2.47(\mathrm{~d}, J=2.4 \mathrm{~Hz}, 0.2 \mathrm{H}) 2.35(\mathrm{~d}, J=2.4 \mathrm{~Hz}, 0.8 \mathrm{H}), 2.05$ (brs, $1 \mathrm{H}), 1.93-1.83(\mathrm{~m}, 1 \mathrm{H}), 1.78-1.71(\mathrm{~m}, 1 \mathrm{H}), 1.27\left(\mathrm{~d}, J=6.6 \mathrm{~Hz}, 3 \mathrm{H} ;{ }^{13} \mathrm{C}\right.$ NMR $(150 \mathrm{MHz}$, $\left.\mathrm{CDCl}_{3}\right) \delta 160.42,82.57,72.17,65.70,44.56,38.51,24.19$; HRMS (EI) Calcd. for $\mathrm{C}_{7} \mathrm{H}_{11} \mathrm{O}_{2} \mathrm{~N}\left[(\mathrm{M})^{+}\right]$141.07898, found 141.07836 .

$\left(3 S^{*}, 5 R^{\star}\right)-3-a m i n o-h e x-1-y n-5-o l, N$-formyl derivative $( \pm)-7 \mathrm{~b}$ :

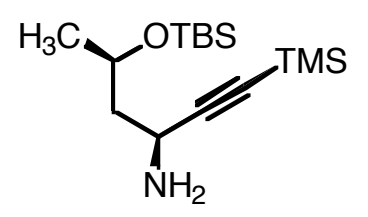

$( \pm)-3 b$

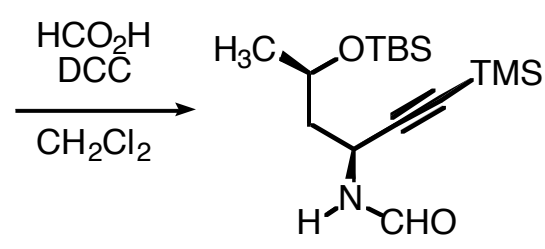

(1)

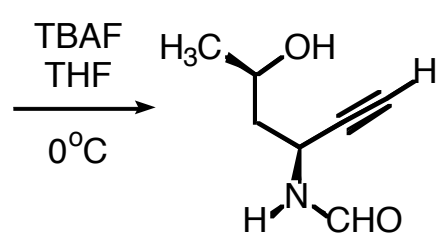

$( \pm)-7 b$

The procedure described above for $N$-formylation was followed using $\mathbf{3} \mathbf{b}$, with purification by column chromatography on silica gel (pet-ether:EtOAc $=8: 2$ ), to provide the $\mathrm{N}$-formyl derivative of $\mathbf{3 b}$ as a white solid (yield $81 \%$ ). Mp $79-81^{\circ} \mathrm{C}$; IR (neat film) 3261, 3029, 2969, 2930, 2896, 2872, 2178, 1681, 1663, 1534, 1473, 1436, 1386, 1251, 1148, 1064, $1004 \mathrm{~cm}^{-1} ;{ }^{1} \mathrm{H}$ NMR $\left(600 \mathrm{MHz}, \mathrm{CDCl}_{3}\right) \delta 8.16(\mathrm{~d}, J=12 \mathrm{~Hz}, 0.2 \mathrm{H}), 8.08$ (s, $0.8 \mathrm{H}), 6.75(\mathrm{~d}, J=7.2 \mathrm{~Hz}, 0.8 \mathrm{H}), 6.18-6.12(\mathrm{~m}, 0.2 \mathrm{H}), 4.98-4.92(\mathrm{~m}, 0.8 \mathrm{H}), 4.41$ (ddd, $J$ $=7.8,7.8,3.6 \mathrm{~Hz}, 0.2 \mathrm{H}), 4.34-4.29(\mathrm{~m}, 0.8 \mathrm{H}), 4.16-4.10(\mathrm{~m}, 0.2 \mathrm{H}), 1.86$ (ddd, $J=14.4$, 
9, 3.6 Hz, 0.4H), 1.82-1.73 (m, 1.6H), $1.21(\mathrm{~d}, J=6.6 \mathrm{~Hz}, 3 \mathrm{H}), 0.92(\mathrm{~s}, 9 \mathrm{H}), 0.16(\mathrm{~s}, 9 \mathrm{H})$, $0.12(\mathrm{~d}, J=13.2 \mathrm{~Hz}, 6 \mathrm{H}) ;{ }^{13} \mathrm{C}$ NMR $\left(150 \mathrm{MHz}, \mathrm{CDCl}_{3}\right) \delta$ 159.68, 103.94, 87.97, 67.21, 43.11, 38.95, 26.13, 24.20, 18.19, 0.090, -3.71, -4.30; HRMS (FAB ${ }^{+}$Calcd. for $\mathrm{C}_{16} \mathrm{H}_{33} \mathrm{O}_{2} \mathrm{NSi}_{2} \mathrm{Li}\left[(\mathrm{M}+\mathrm{Li})^{+}\right]$334.2210, found 334.2209. Anal. Calcd. for $\mathrm{C}_{16} \mathrm{H}_{33} \mathrm{O}_{2} \mathrm{NSi}_{2}$ : C, 58.66; H, 10.15; N, 4.28; Found: C, 58.79; H, 10.15; N, 4.27.

The general procedure for desilylation was followed, and purification by column chromatography on silica gel $\left(\mathrm{CH}_{2} \mathrm{Cl}_{2}: \mathrm{CH}_{3} \mathrm{OH}=98: 2\right)$ provided $\mathbf{7 b}$ as an oil (yield $90 \%$ ). IR (neat film) 3287, 3039, 2965, 2925, 2876, 2859, 2118, 1666, 1530, 1461, 13841260 , $1093 \mathrm{~cm}^{-1} ;{ }^{1} \mathrm{H}$ NMR $\left(600 \mathrm{MHz}, \mathrm{CDCl}_{3}\right) \delta 8.19(\mathrm{~s}, 1 \mathrm{H}), 6.42$ (brs, $\left.1 \mathrm{H}\right), 5.08-5.02(\mathrm{~m}, 1 \mathrm{H})$, 4.08-3.98 (m, 1H), $2.97(\mathrm{brs}, 1 \mathrm{H}), 2.34(\mathrm{~d}, J=3.6 \mathrm{~Hz}, 1 \mathrm{H}) 1.86-1.75(\mathrm{~m}, 2 \mathrm{H}), 1.25(\mathrm{~d}, J$ $=9.6 \mathrm{~Hz}, 3 \mathrm{H}) ;{ }^{13} \mathrm{C}$ NMR $\left(150 \mathrm{MHz}, \mathrm{CDCl}_{3}\right) \delta 161.04,82.22,72.06,64.48,44.41,38.28$, 23.43; HRMS (EI) Calcd. for $\mathrm{C}_{7} \mathrm{H}_{11} \mathrm{O}_{2} \mathrm{~N}\left[(\mathrm{M})^{+}\right] 141.07898$, found 141.07845 .

$\left(3 R^{\star}, 5 R^{\star}\right)-3-($ methylamino)-hex-1-yn-5-ol, $N$-formyl derivative ( \pm )-8a:

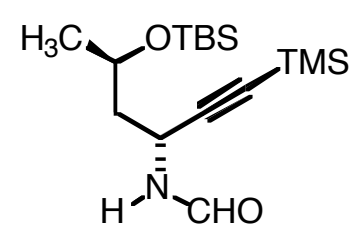

$\mathrm{N}$-formyl derivative of $( \pm)-3 a$
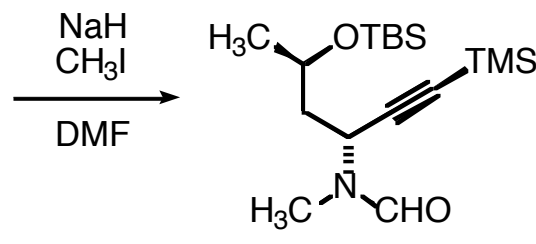

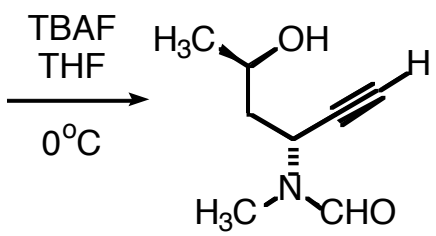

$( \pm)-8 a$

To a stirred solution of the above-described $N$-formyl derivative of $3 \mathrm{a}(1.2 \mathrm{~g}, 3.7 \mathrm{mmol})$ in DMF $(18 \mathrm{~mL})$ at room temperature was carefully added $\mathrm{NaH}(0.294 \mathrm{~g}, 7.4 \mathrm{mmol})$. The reaction was stirred $15 \mathrm{~min}$, at which time $\mathrm{CH}_{3}$ I $(3.151 \mathrm{~g}, 3.4 \mathrm{~mL}, 22.2 \mathrm{mmol})$ was added dropwise. The reaction progress was followed by TLC. The reaction was quenched with $\mathrm{H}_{2} \mathrm{O}(60 \mathrm{~mL})$. The organic layer was separated and the aqueous layer was extracted with EtOAc $(3 \times 50 \mathrm{~mL})$. The combined organic layers were washed with $\mathrm{NaHCO}_{3}$, dried over $\mathrm{Na}_{2} \mathrm{SO}_{4}$, and evaporated under reduced pressure. The residual oil was purified by column chromatography on silica gel (pet-ether:EtOAc $=8: 2$ ) to yield $N$ formyl- $N$-methyl derivative of $\mathbf{3 a}$ as an oil $(0.8 \mathrm{~g}, 80 \%$ yield). IR (neat film) 3584, 3310, $3241,2956,2929,2857,2114,1681,1472,1464,1404,1380,1003 \mathrm{~cm}^{-1} ;{ }^{1} \mathrm{H}$ NMR $(600$ $\left.\mathrm{MHz}, \mathrm{CDCl}_{3}\right) \delta 8.14(\mathrm{~s}, 0.7 \mathrm{H}), 8.01(\mathrm{~s}, 0.3 \mathrm{H}), 5.41$ (ddd, $\left.J=13.2,9,4.2 \mathrm{~Hz}, 0.3 \mathrm{H}\right), 4.51$ 
(ddd, $J=16.2,6.6,3.6 \mathrm{~Hz}, 0.7 \mathrm{H}), 4.02$ (qdd, $J=9,9,4.8 \mathrm{~Hz}, 0.7 \mathrm{H}), 3.99-3.91(\mathrm{~m}$, $0.3 \mathrm{H}), 2.95(\mathrm{~s}, 0.9 \mathrm{H}), 2.89(\mathrm{~s}, 2.1 \mathrm{H}), 2.42(\mathrm{~d}, J=3.6 \mathrm{~Hz}, 0.7 \mathrm{H}), 2.29(\mathrm{~d}, J=3 \mathrm{~Hz}, 0.3 \mathrm{H})$, $1.90(\mathrm{ddd}, J=19.8,16.2,4.8 \mathrm{~Hz}, 0.7 \mathrm{H}), 1.83-1.75(\mathrm{~m}, 0.3 \mathrm{H}), 1.73-1.63(\mathrm{~m}, 1 \mathrm{H}), 1.21$ $(\mathrm{d}, J=9 \mathrm{~Hz}, 0.9 \mathrm{H}), 1.20(\mathrm{~d}, J=9 \mathrm{~Hz}, 2.1 \mathrm{H}), 0.92(\mathrm{~s}, 9 \mathrm{H}), 0.100(\mathrm{~d}, J=4.2 \mathrm{~Hz}, 6 \mathrm{H}) ;{ }^{13} \mathrm{C}$ NMR $\left(150 \mathrm{MHz}, \mathrm{CDCl}_{3}\right) \delta 162.30,80.52,74.45,65.43,48.41,44.63,30.71,26.01$, 24.24, 18.15, -3.86, -4.76; HRMS (FAB ${ }^{+}$Calcd. for $\mathrm{C}_{14} \mathrm{H}_{27} \mathrm{O}_{2} \mathrm{NSi}\left[(\mathrm{M}+\mathrm{Li})^{+}\right]$ 276.1971, found 276.1977. Anal. Calcd. for $\mathrm{C}_{14} \mathrm{H}_{27} \mathrm{O}_{2} \mathrm{NSi}$ : C, 62.40; $\mathrm{H}, 10.10 ; \mathrm{N}, 5.20$; Found: $\mathrm{C}, 62.31 ; \mathrm{H}, 10.27 ; \mathrm{N}, 5.25$.

The general procedure for desilylation was followed, and purification by column chromatography on silica gel (pet-ether:EtOAc $=8: 2$ ) provided $8 \mathbf{a}$ as an oil (yield $90 \%$ ). IR (neat film) 3409, 3289, 2967, 2928, 2880, 2115, 1665, 1485, 1404, 15, 1313 1260, 1241, 1118, 1129, $1079 \mathrm{~cm}^{-1} ;{ }^{1} \mathrm{H}$ NMR $\left(600 \mathrm{MHz}, \mathrm{CDCl}_{3}\right) \delta 8.12(\mathrm{~s}, 0.7 \mathrm{H}), 7.95(\mathrm{~s}$, $0.3 \mathrm{H}$ ), 5.40 (ddd, $J=11.4,6.6,2.4 \mathrm{~Hz}, 0.3 \mathrm{H}$ ), 4.60 (ddd, $J=10.2,5.4,2.4 \mathrm{~Hz}, 0.7 \mathrm{H}$ ), 4.02-3.96 (m, 0.7H), 3.95-3.88 (m, 0.3H), $2.96(\mathrm{~s}, 0.91 \mathrm{H}), 2.85,(\mathrm{~s}, 2.1 \mathrm{H}), 2.41(\mathrm{~d}, J=$ $2.4 \mathrm{~Hz}, 0.7 \mathrm{H}$ ), 2.32 (d, $J=2.4 \mathrm{~Hz}, 0.3 \mathrm{H}$ ), 1.89 (ddd, $J=13.2,10.2,2.4 \mathrm{~Hz}, 0.7 \mathrm{H}) 1.81$ (ddd, $J=13.2,9,3.6 \mathrm{~Hz}, 0.3 \mathrm{H}) 1.69-1.60(\mathrm{~m}, 1 \mathrm{H}), 1.23(\mathrm{~d}, J=6.6 \mathrm{~Hz}, 2.1 \mathrm{H}), 1.21(\mathrm{~d}, J=$ $6.6 \mathrm{~Hz}, 0.9 \mathrm{H}) ;{ }^{13} \mathrm{C} \mathrm{NMR}\left(150 \mathrm{MHz}, \mathrm{CDCl}_{3}\right) \delta$ 162.53, 80.30, 73.56, 64.88, 48.54, 43.49, 30.89, 24.31; HRMS (FAB ${ }^{+}$) Calcd. for $\mathrm{C}_{8} \mathrm{H}_{13} \mathrm{O}_{2} \mathrm{NLi}\left[(\mathrm{MLi})^{+}\right]$162.1106, found 162.1104.

\section{$\left(3 S^{*}, 5 R^{\star}\right)$-3-(methylamino)-hex-1-yn-5-ol, $N$-formyl derivative $( \pm)-8 \mathrm{~b}$ :}

The procedure described above for $N$-methylation of the $N$-formyl derivative of 3a was followed with the $N$-formyl derivative of $3 \mathbf{b}$, with purification by column chromatography on silica gel (pet-ether:EtOAc $=8: 2$ ), to provide $N$-formyl- $N$-methyl derivative as an oil (yield 75\%). IR (neat film) 3584, 3310, 3241, 2955, 2930, 2886, 2857, 2115, 1681, 1472, 1463, 1403, 1382, 1255, 1144, 1095, 1066, 1034, $1003 \mathrm{~cm}^{-1} ;{ }^{1} \mathrm{H}$ NMR $(600 \mathrm{MHz}$, $\left.\mathrm{CDCl}_{3}\right) \delta 8.10(\mathrm{~s}, 0.25 \mathrm{H}), 8.01(\mathrm{~s}, 0.75 \mathrm{H}), 5.28(\mathrm{dd}, J=7.2,7.2 \mathrm{~Hz}, 0.25 \mathrm{H}), 4.53(\mathrm{dd}, J=$ 7.2, 7.2 Hz, 0.75H), 3.89 (qdd, $J=6,6,5.4 \mathrm{~Hz}, 0.25 \mathrm{H}), 3.78-3.72(\mathrm{~m}, 0.75 \mathrm{H}), 2.95(\mathrm{~d}, J$ $=0.6 \mathrm{~Hz}, 0.75 \mathrm{H}), 2.86(\mathrm{~d}, J=0.6 \mathrm{~Hz}, 2.25 \mathrm{H}), 2.39(\mathrm{dd}, J=1.2,1.2 \mathrm{~Hz}, 0.75 \mathrm{H}), 2.29(\mathrm{~d}$, $J=1.2 \mathrm{~Hz}, 0.25 \mathrm{H}$ ), 1.91 (ddd, $J=14.4,10.2,7.2 \mathrm{~Hz}, 0.5 \mathrm{H}), 1.88-1.81(\mathrm{~m}, 1 \mathrm{H}), 1.71$ (ddd, $J=14.4,6,6 \mathrm{~Hz}, 0.5 \mathrm{H}$ ), 1.20 (app.dd, $J=6,1.2 \mathrm{~Hz}, 3 \mathrm{H}$ ), 0.89 (d, $J=0.6 \mathrm{~Hz}, 9 \mathrm{H}$ ), 0.06 (app.t, $J=7.2 \mathrm{~Hz}, 6 \mathrm{H}),{ }^{13} \mathrm{C}$ NMR $\left(150 \mathrm{MHz}, \mathrm{CDCl}_{3}\right) \delta 162.80,81.32,73.43,64.55$, 
$47.15,43.43,30.51,26.13,23.98,18.22,-3.34,-4.38$; HRMS $\left(F_{A B}^{+}\right)$Calcd. for $\mathrm{C}_{14} \mathrm{H}_{28} \mathrm{O}_{2} \mathrm{NSi}\left[(\mathrm{M}+\mathrm{H})^{+}\right]$270.1889, found 270.1877. Anal. Calcd. for $\mathrm{C}_{14} \mathrm{H}_{27} \mathrm{O}_{2} \mathrm{NSi}$ : C, 62.40; H, 10.10; N, 5.20; Found: C, 62.55; H, 10.10; N, 5.23.

The general procedure for desilylation was followed, and purification by column chromatography on silica gel (pet-ether:EtOAc $=8: 2$ ) provided $\mathbf{8 b}$ as an oil (yield $98 \%$ ). IR (neat film) 3414, 3296, 2967, 2927, 2879, 2116, 1665, 1530, 1457, 1428, 1405, 1301 1131, $1081 \mathrm{~cm}^{-1} ;{ }^{1} \mathrm{H}$ NMR $\left(600 \mathrm{MHz}, \mathrm{CDCl}_{3}\right) \delta 7.94$ (s, 0.3H), 7.92 (s, 0.7H), 5.20 (ddd, $J=11.4,1.8,1.8 \mathrm{~Hz}, 0.7 \mathrm{H}$ ), 4.50 (ddd, $J=13.2,4.2,2.4 \mathrm{~Hz}, 0.3 \mathrm{H}$ ), 3.49 (brs, 0.7H), 3.36 (ddd, $J=10.2,6,2.4 \mathrm{~Hz}, 0.3 \mathrm{H}$ ), $2.81(\mathrm{~s}, 2.1 \mathrm{H}), 2.69(\mathrm{~s}, 0.9 \mathrm{H}), 2.22(\mathrm{~d}, J=2.4 \mathrm{~Hz}$, $0.3 \mathrm{H}$ ), $2.21(\mathrm{~d}, J=2.4 \mathrm{H}, 0.7 \mathrm{H}$ ), 1.75 (ddd, $J=13.2,13.2,2.4 \mathrm{~Hz}, 0.3 \mathrm{H}$ ), 1.61 (ddd, $J=$ 11.4,11.4, $2.4 \mathrm{~Hz}, 0.7 \mathrm{H}), 1.59$ (ddd, $J=10.2,10.2,4.2 \mathrm{~Hz}, 1 \mathrm{H}), 1.06(\mathrm{~d}, J=6 \mathrm{~Hz}, 0.9 \mathrm{H}$ ), $1.02(\mathrm{~d}, J=6 \mathrm{~Hz}, 2.1 \mathrm{H}) ;{ }^{13} \mathrm{C}$ NMR $\left(150 \mathrm{MHz}, \mathrm{CDCl}_{3}\right) \delta$ 163.45, 80.22, 73.92, 62.88, 47.27, 42.45, 30.80, 22.27; HRMS (FAB ${ }^{+}$Calcd. for $\mathrm{C}_{8} \mathrm{H}_{13} \mathrm{O}_{2} \mathrm{NLi}\left[(\mathrm{MLi})^{+}\right]$162.1106, found 162.1108 .

$\left(3 R^{\star}, 5 R^{\star}\right)-3$-amino-hex-1-yn-5-ol, $N, N-B_{0 c}$ derivative ( \pm )-9a:

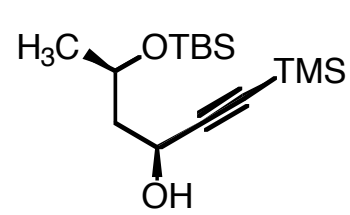

$( \pm)-2 a$

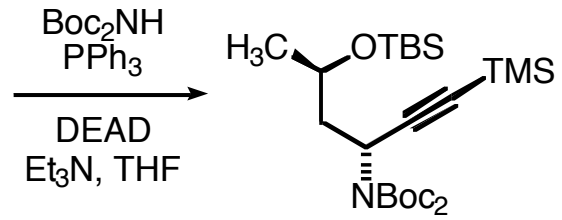

DEAD
$t_{3} \mathrm{~N}, \mathrm{THF}$

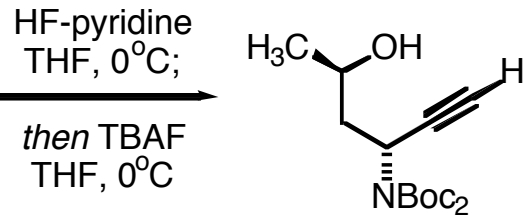

$( \pm)-9 a$

A $50 \mathrm{~mL}$ flask was charged with triphenylphosphine, $\left(\mathrm{Ph}_{3} \mathrm{P}, 1.312 \mathrm{~g}, 5 \mathrm{mmol}\right)$ and a stir bar. Vacuum ( $\leq 0.5 \mathrm{mbar}$ ) was applied and the flask was heated to $50{ }^{\circ} \mathrm{C}$ overnight. At the same time a $10 \mathrm{~mL}$ flask was charged with di-tert-butyl iminodicarboxylate $\left(\mathrm{HN}(\mathrm{Boc})_{2}, 1.740 \mathrm{~g}, 8 \mathrm{mmol}\right)$. Vacuum ( $\leq 0.5 \mathrm{mbar}$ ) was applied and the flask was heated to $50{ }^{\circ} \mathrm{C}$ overnight. The two flasks were brought to room temperature and the vacuum was replaced with a flow of argon. Solvent (THF, $10 \mathrm{~mL}$ ) was added to the $\mathrm{HN}(\mathrm{Boc})_{2}$ and the solution was transferred by syringe to the $50 \mathrm{~mL}$ flask containing the $\mathrm{Ph}_{3} \mathrm{P}$ to which an additional $10 \mathrm{~mL}$ THF was added. The reaction mixture was reduced to $0{ }^{\circ} \mathrm{C}$. A separate $10 \mathrm{~mL}$ flask was charged with $2 \mathrm{a}(0.601 \mathrm{~g}, 2 \mathrm{mmol})$ in THF $(2 \mathrm{~mL})$ 
and $\mathrm{Et}_{3} \mathrm{~N}(0.85 \mathrm{~mL}, 6 \mathrm{mmol})$ at $0{ }^{\circ} \mathrm{C}$ and the solution was allowed to stir for $30 \mathrm{~min}$ at which time the solution was transferred by syringe to the reaction flask containing $\mathrm{Ph}_{3} \mathrm{P}$ and $\mathrm{HN}(\mathrm{Boc})_{2}$ in solution at $0{ }^{\circ} \mathrm{C}$. To this reaction mixture was added diethyl azodicarboxylate, (DEAD, $2.73 \mathrm{~mL}, 6 \mathrm{mmol}$ ) dropwise over 30 minutes. Immediately upon completion of the addition of DEAD the reaction vessel was fitted with a condenser and heated to $50{ }^{\circ} \mathrm{C}$ for $4 \mathrm{~h}$. The reaction was quenched by evaporation of solvent under reduced pressure. Purification of the material by flash column chromatography on silica gel (pet-ether:EtOAc $=98: 2$ ) afforded the $\mathrm{N}, \mathrm{N}-\mathrm{Boc}_{2}$ product as an oil (0.454 g, 45\% yield). Mp 71-72 ${ }^{\circ} \mathrm{C}$; IR (neat film) 2960, 2931, 2899, 2857, 2176 , 1747, 1710, 1473, 1461, 1368, 1345, 1251, 1159, 1119, $1006 \mathrm{~cm}^{-1} ;{ }^{1} \mathrm{H}$ NMR $(400 \mathrm{MHz}$, $\left.\mathrm{CDCl}_{3}\right) \delta 5.18(\mathrm{dd}, J=10.4,5.2 \mathrm{~Hz}, 1 \mathrm{H}), 3.99(\mathrm{dqd}, J=10,6,3.2 \mathrm{~Hz}, 1 \mathrm{H}), 2.18$ (ddd, $J$ $=13,10.4,3.2 \mathrm{~Hz}, 1 \mathrm{H}), 1.74$ (ddd, $J=13,10,5.2 \mathrm{~Hz}, 1 \mathrm{H}), 1.52(\mathrm{~s}, 18 \mathrm{H}), 1.17$ (d, J = 6 $\mathrm{Hz}, 3 \mathrm{H}), 0.91$ (s, 9H), 0.15 (s, 9H), $0.10(\mathrm{~d}, J=14 \mathrm{~Hz}, 6 \mathrm{H}) ;{ }^{13} \mathrm{C} \mathrm{NMR}\left(100 \mathrm{MHz}, \mathrm{CDCl}_{3}\right)$ $\delta$ 152.09, 103.72, 88.41, 82.75, 66.06, 47.13, 44.38, 28.27, 26.15, 24.42, 18.27, 0.16, -4.09, -4.57; HRMS $\left(\mathrm{FAB}^{+}\right)$Calcd. for $\mathrm{C}_{25} \mathrm{H}_{48} \mathrm{O}_{5} \mathrm{NSi}_{2} \mathrm{Li}\left[(\mathrm{M}+\mathrm{Li})^{+}\right] 506.3309$, found 506.3288. Anal. Calcd. for $\mathrm{C}_{25} \mathrm{H}_{48} \mathrm{O}_{5} \mathrm{NSi}_{2}$ : C, 60.20; H, 9.70; N, 2.81; Found C, 60.35; $\mathrm{H}, 9.93 ; \mathrm{N}, 2.75$.

The O-TBS was removed by dropwise addition of HF-pyridine (70:30, $0.50 \mathrm{~mL}, 19.3$ $\mathrm{mmol})$ to a stirred solution of the above-described $\mathrm{N}, \mathrm{N}-\mathrm{Boc}_{2}$ product $(0.3200 \mathrm{~g}, 0.65$ $\mathrm{mmol})$ in THF $(3 \mathrm{~mL})$ at $0{ }^{\circ} \mathrm{C}$. The reaction was monitored by TLC and if starting material remained, additional HF-py was added as needed. The reaction was quenched by addition of $\mathrm{Et}_{2} \mathrm{O}\left(10 \mathrm{~mL}\right.$ per $\mathrm{mL} \mathrm{THF}$ ) followed by $\mathrm{NaHCO}_{3}(1 \mathrm{~mL}$ per $\mathrm{mL}$ $\mathrm{THF}$ ) at $0{ }^{\circ} \mathrm{C}$. After $30 \mathrm{~min}$ the reaction mixture was allowed to come to room temperature and stirred $30 \mathrm{~min}$. The organic layer was separated, washed with a saturated solution of $\mathrm{NaCl}(20 \mathrm{~mL})$, dried over $\mathrm{Na}_{2} \mathrm{SO}_{4}$, and evaporated under reduced pressure. The residual oil was purified by column chromatography on silica gel (petether:EtOAc $=8: 2)$ to yield the secondary alcohol as a white solid $(0.180 \mathrm{~g}, 73 \%$ yield $)$. Mp 71-72 ${ }^{\circ} \mathrm{C}$; IR (neat film) 3484, 2977, 2932, 2176, 1745, 1706, 1478, 1457, 1368, 1249, 1150, $1122 \mathrm{~cm}^{-1} ;{ }^{1} \mathrm{H}$ NMR $\left(400 \mathrm{MHz}, \mathrm{CDCl}_{3}\right) \delta 5.29(\mathrm{dd}, J=9.2,5.2 \mathrm{~Hz}, 1 \mathrm{H})$, 4.04-3.91 (brs, 1H), 2.21 (ddd, $J=13.6,9.2,2.8 \mathrm{~Hz}, 1 \mathrm{H}$ ), 1.81 (ddd, $J=13.6,9.6,5.2$ $\mathrm{Hz}, 1 \mathrm{H}), 1.52(\mathrm{~s}, 18 \mathrm{H}), 1.25(\mathrm{~d}, J=6.4 \mathrm{~Hz}, 3 \mathrm{H}), 0.16(\mathrm{~s}, 9 \mathrm{H}) ;{ }^{13} \mathrm{C} \mathrm{NMR}(100 \mathrm{MHz}$, 
$\left.\mathrm{CDCl}_{3}\right) \delta 152.13,103.39,88.52,83.10,65.74,46.74,44.22,28.24,24.01,0.13 ; \mathrm{HRMS}$ $\left(\mathrm{FAB}^{+}\right)$Calcd. for $\mathrm{C}_{19} \mathrm{H}_{35} \mathrm{O}_{5} \mathrm{NSiLi}\left[(\mathrm{MLi})^{+}\right]$392.2445, found 392.2445. Anal. Calcd. for $\mathrm{C}_{19} \mathrm{H}_{35} \mathrm{O}_{5} \mathrm{~N}$ : C, 59.19; H, 9.15; N, 3.63; Found: C, 59.53; H, 9.22; N, 3.62.

The alkynyl-TMS group was then removed by dropwise addition of TBAF (1M in THF, $0.46 \mathrm{~mL} 0.46 \mathrm{mmol}$ ) to a stirred solution of the above-described secondary alcohol $(0.090 \mathrm{~g}, 0.23 \mathrm{mmol})$ and acetic acid $(0.01 \mathrm{~mL}, 0.23 \mathrm{mmol})$ in THF $(2 \mathrm{~mL})$ at $0{ }^{\circ} \mathrm{C}$. The reaction was monitored by TLC and if starting material remained, additional TBAF was added as needed. The reaction was quenched with $\mathrm{H}_{2} \mathrm{O}(10 \mathrm{~mL})$. The organic layer was separated and the aqueous layer was extracted with EtOAc $(3 \times 15 \mathrm{~mL})$. The combined organic layers were washed with $\mathrm{NaHCO}_{3}$, dried over $\mathrm{Na}_{2} \mathrm{SO}_{4}$, and evaporated under reduced pressure. The residual oil was purified by column chromatography on silica gel (pet-ether:EtOAc $=8: 2)$ to yield $9 \mathrm{a}$ as an oil $(0.071 \mathrm{~g}, 96 \%$ yield). IR (neat film) 3439, 3312, 2977, 2932, 2121, 1743, 1704, 1458, 1369,1246, 1151', $1125, \mathrm{~cm}^{-1} ;{ }^{1} \mathrm{H}$ NMR $\left(400 \mathrm{MHz}, \mathrm{CDCl}_{3}\right) \delta 5.31$ (ddd, $J=9.6,5.2,2.8 \mathrm{~Hz}, 1 \mathrm{H}$ ), 4.02 (dqd, 9.6, 6.4, 3.2 Hz, 1H), 2.34 (d, $J=2.8 \mathrm{~Hz}, 1 \mathrm{H}$ ), 2.24 (ddd, $J=13.2,9.6,3.2$ $\mathrm{Hz}, 1 \mathrm{H}$ ), 1.81 (ddd, $J=13.2,9.6,5.2 \mathrm{~Hz}, 1 \mathrm{H}), 1.52(\mathrm{~s}, 18 \mathrm{H}), 1.25$ (d, $J=6.4 \mathrm{~Hz}, 3 \mathrm{H}$ ); ${ }^{13} \mathrm{C}$ NMR $\left(100 \mathrm{MHz}, \mathrm{CDCl}_{3}\right) \delta 152.29,83.27,81.77,65.56,45.92,43.64,28.23,24.09$; HRMS ( $\left.\mathrm{FAB}^{+}\right)$Calcd. for $\mathrm{C}_{16} \mathrm{H}_{27} \mathrm{O}_{5} \mathrm{NLi}\left[(\mathrm{MLi})^{+}\right] 320.2049$, found 320.2041. Anal. Calcd. for $\mathrm{C}_{16} \mathrm{H}_{27} \mathrm{O}_{5} \mathrm{~N}$ : C, 61.32; $\mathrm{H}, 8.68 ; \mathrm{N}, 4.47$; Found: $\mathrm{C}, 61.08 ; \mathrm{H}, 8.60 ; \mathrm{N}, 4.19$.

\section{$\left(3 S^{*}, 5 R^{\star}\right)$-3-amino-hex-1-yn-5-ol, $N, N-B_{0} c_{2}$ derivative ( \pm )-9b:}

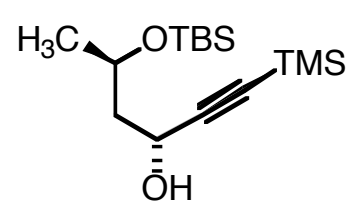

$( \pm)-2 b$

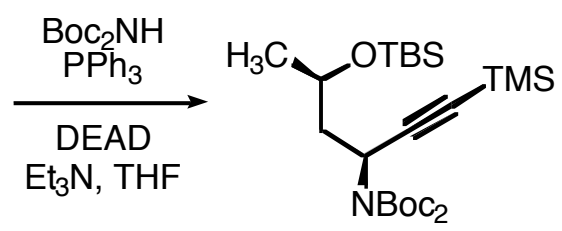

$t_{3} \mathrm{~N}, \mathrm{THF}$

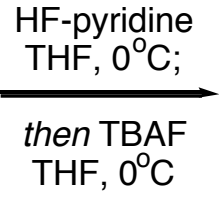

then TBAF

$\mathrm{THF}, 0^{\circ} \mathrm{C}$

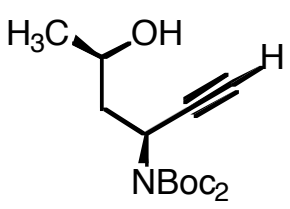

$( \pm)-9 b$

Substitution from $\mathbf{2 b}$ with $\mathrm{Boc}_{2} \mathrm{NH}$ was accomplished as described from $\mathbf{2 a}$, and purification by column chromatography on silica gel (pet-ether:EtOAc $=98: 2$ ) to provided the $\mathrm{N}, \mathrm{N}-\mathrm{Boc}_{2}$ product as an oil (yield 50\%). IR (neat film) 2959, 2931, 2857, $2177,1750,1711,1473,1461,1368,1345,1251,1150,1121,1006 \mathrm{~cm}^{-1} ;{ }^{1} \mathrm{H}$ NMR (400 $\left.\mathrm{MHz}, \mathrm{CDCl}_{3}\right) \delta 5.02$ (dd, $J=8,5.2 \mathrm{~Hz}, 1 \mathrm{H}$ ); 3.92 (qdd, $J=6,5.2,3.2 \mathrm{~Hz}, 1 \mathrm{H}$ ), 2.28 (ddd, 
$J=13.2,5.2,3.2 \mathrm{~Hz}, 1 \mathrm{H}), 1.81$ (ddd, $J=13.2,8,5.2 \mathrm{~Hz}, 1 \mathrm{H}), 1.52(\mathrm{~s}, 18 \mathrm{H}), 1.20(\mathrm{~d}, J=$ $6 \mathrm{~Hz}, 3 \mathrm{H}), 0.90$ (s, 9H), $0.15(\mathrm{~s}, 9 \mathrm{H}), 0.076(\mathrm{~s}, 6 \mathrm{H}) ;{ }^{13} \mathrm{C} \mathrm{NMR}\left(100 \mathrm{MHz}, \mathrm{CDCl}_{3}\right) \delta$ 151.94, 104.00, 87.71, 82.93, 66.25, 46.20, 44.88, 28.27, 26.16, 23.22, 18.36, 0.13, -4.30, -4.55; Calcd. for $\mathrm{C}_{25} \mathrm{H}_{48} \mathrm{O}_{5} \mathrm{NSi}_{2} \mathrm{Li}\left[(\mathrm{M}+\mathrm{Li})^{+}\right]$506.3309, found 506.3323. Anal. Calcd. for $\mathrm{C}_{25} \mathrm{H}_{48} \mathrm{O}_{5} \mathrm{NSi}_{2}$ : C, 60.20; $\mathrm{H}, 9.70 ; \mathrm{N}, 2.81$; Found C, 60.33; H, 9.63; N, 2.75.

O-desilylation as described above and purification by column chromatography on silica gel (pet-ether:EtOAc $=98: 2$ ) provided the secondary alcohol as a clear oil (yield $81 \%$ ). IR (neat film) 3524, 2977, 2934, 2177, 1746, 1709, 1478, 1458, 1369,1250, 1150, 1121 $\mathrm{cm}^{-1} ;{ }^{1} \mathrm{H}$ NMR $\left(400 \mathrm{MHz}, \mathrm{CDCl}_{3}\right) \delta 5.24$ (dd, $\left.J=10.8,10.4 \mathrm{~Hz}, 1 \mathrm{H}\right), 3.72$ (qdd, $J=6$, 5.8, $2.8 \mathrm{~Hz}, 1 \mathrm{H}$ ), 3.06 (d, $J=5.4 \mathrm{~Hz}, 1 \mathrm{H}$ ), 2.20 (ddd, $J=13.6,10.8,2.8 \mathrm{~Hz}, 1 \mathrm{H}$ ), 1.85 (ddd, $J=13.6,10.4,5.8 \mathrm{~Hz}, 1 \mathrm{H}), 1.53(\mathrm{~s}, 18 \mathrm{H}), 1.21(\mathrm{~d}, J=6 \mathrm{~Hz}, 3 \mathrm{H}) ;{ }^{13} \mathrm{C}$ NMR $(100$ $\left.\mathrm{MHz}, \mathrm{CDCl}_{3}\right) \delta 152.69,103.73,88.09,83.57,64.13,46.26,44.65,28.21,22.80,0.07$; HRMS (FAB ${ }^{+}$) Calcd. for $\mathrm{C}_{19} \mathrm{H}_{35} \mathrm{O}_{5} \mathrm{NLi}\left[(\mathrm{MLi})^{+}\right]$392.2445, found 392.2457. Anal. Calcd. for $\mathrm{C}_{19} \mathrm{H}_{35} \mathrm{O}_{5} \mathrm{~N}$ : C, 59.19; H, 9.15; N, 3.63; Found: C, 59.88; H, 8.99; N, 3.60.

Removal of the alkynyl-TMS substituent was accomplished as described above, with purification by column chromatography on silica gel (pet-ether:EtOAc $=8: 2$ ) to provide 9b as a white solid (yield 86\%). Mp 67-68 ${ }^{\circ} \mathrm{C}$; IR (neat film) 3524, 3272, 2979, 2933, $2121,1739,1705,1478,1458,1369,1249,1150,1122, \mathrm{~cm}^{-1} ;{ }^{1} \mathrm{H}$ NMR $(400 \mathrm{MHz}$, $\left.\mathrm{CDCl}_{3}\right) \delta 5.21$ (ddd, $\left.J=10.8,4.4,2.8 \mathrm{~Hz}, 1 \mathrm{H}\right), 3.73(\mathrm{dqd}, J=10,6,2.8 \mathrm{~Hz}, 1 \mathrm{H}), 2.98(\mathrm{~d}$, $J=4.4 \mathrm{~Hz}, 1 \mathrm{H}$ ), 2.34 (d, $J=2.8 \mathrm{~Hz}, 1 \mathrm{H}$ ), 2.26 (ddd, $J=14,10.8,2.8 \mathrm{~Hz}, 1 \mathrm{H}$ ), 1.84 (ddd, $J=21,15,6.6 \mathrm{~Hz}, 1 \mathrm{H}), 1.53(\mathrm{~s}, 18 \mathrm{H}), 1.22(\mathrm{~d}, J=9 \mathrm{~Hz}, 3 \mathrm{H}) ;{ }^{13} \mathrm{C}$ NMR $(100 \mathrm{MHz}$, $\left.\mathrm{CDCl}_{3}\right) \delta$ 152.88, 83.75, 82.10, 71.62, 63.90, 45.38, 44.08, 28.19, 22.79; HRMS (FAB ${ }^{+}$) Calcd. for $\mathrm{C}_{16} \mathrm{H}_{27} \mathrm{O}_{5} \mathrm{NLi}\left[(\mathrm{MLi})^{+}\right] 320.2049$, found 320.2036. Anal. Calcd. for $\mathrm{C}_{16} \mathrm{H}_{27} \mathrm{O}_{5} \mathrm{~N}$ : C, 61.32; H, 8.68; N, 4.47; Found: C, 61.35; H, 8.73; N, 4.39. 
General procedure for tungsten-catalyzed cycloisomerization synthesis of $\mathbf{N}$-acyl3-amino glycals (10-15):

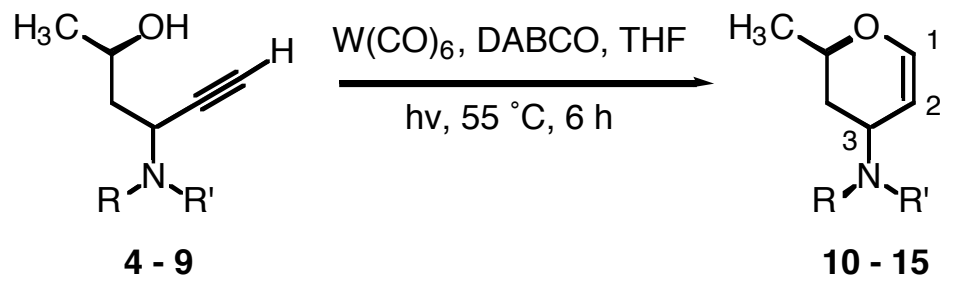

A $10 \mathrm{~mL}$ Schlenk flask was charged with $\mathrm{N}$-acyl-3-amino-hex-1-yn-5-ol (4-9) (1 mmol) and the contents were azeotropically dried with benzene $(2 \times 0.5 \mathrm{~mL})$. THF $(2.5 \mathrm{~mL})$ was added to the reaction flask, followed by $W(C O)_{6}(0.0351 \mathrm{~g}, 0.1 \mathrm{mmol})$ and $1,4-$ diazabicyclo[2.2.2] octane (DABCO, $0.224 \mathrm{~g}, 2 \mathrm{mmol}$ ). The flask was fitted with a reflux condenser and carefully degassed by three cycles of the standard freeze-thaw method. The reaction flask was placed under an atmosphere of argon and irradiated at $350 \mathrm{~nm}$ (Rayonet Photoreactor) at $55{ }^{\circ} \mathrm{C}$ for $6 \mathrm{~h}$. Evaporation of solvent followed by column chromatography on silica gel provided the product glycals 10-15.

$\left(3 R^{*}, 5 R^{*}\right)-N$-Boc-3-amino-glycal (10a)

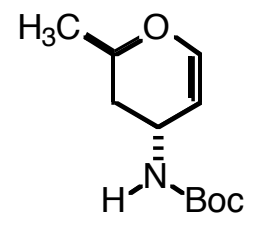

The general procedure was followed using $4 \mathrm{a}(0.123 \mathrm{~g}, 0.58 \mathrm{mmol}), \mathrm{W}(\mathrm{CO})_{6},(0.0102 \mathrm{~g}$, $0.03 \mathrm{mmol})$, DABCO $(0.131 \mathrm{~g}, 0.12 \mathrm{mmol})$. Purification by column chromatography on silica gel (pet-ether:EtOAc $=95: 5)$ provided glycal $(10 \mathrm{a})$ as a white solid $(0.108 \mathrm{~g}$, yield $88 \%$ ). Mp 101-103 ${ }^{\circ} \mathrm{C}$; IR (neat film) 3368, 3130, 2977, 2928, 2868, 2130, 1678, 1645, 1509, 1398, $1241 \mathrm{~cm}^{-1} ;{ }^{1} \mathrm{H}$ NMR $\left(600 \mathrm{MHz}, \mathrm{CDCl}_{3}\right) \delta 6.49(\mathrm{~d}, J=6 \mathrm{~Hz}, 1 \mathrm{H}), 4.74$ (ddd, $J$ $=6,5.7,1.8 \mathrm{~Hz}, 1 \mathrm{H}$ ), 4.07 (brs, $1 \mathrm{H}$ ), 3.86 (qdd, $J=6,4.2,1.8 \mathrm{~Hz}, 1 \mathrm{H}$ ), 1.90 (d, $J=13.4$ $\mathrm{Hz}, 1 \mathrm{H}$ ), 1.65 (ddd, $J=13.4,12.9,4.2 \mathrm{~Hz}, 1 \mathrm{H}), 1.44(\mathrm{~s}, 9 \mathrm{H}), 1.30(\mathrm{~d}, J=6 \mathrm{~Hz}, 3 \mathrm{H}) ;{ }^{13} \mathrm{C}$ NMR $\left(150 \mathrm{MHz}, \mathrm{CDCl}_{3}\right) \delta 154.84,147.33,100.18,79.62,68.20,41.21,37.07,28.61$, 20.98; HRMS (EI) Calcd. for $\mathrm{C}_{11} \mathrm{H}_{19} \mathrm{O}_{3} \mathrm{~N}\left[(\mathrm{M})^{+}\right]$213.13649, found 220.13590. Anal. Calcd. for $\mathrm{C}_{11} \mathrm{H}_{19} \mathrm{O}_{3} \mathrm{~N}$ : C, 61.95; $\mathrm{H}, 9.00 ; \mathrm{N}, 6.57$; Found: $\mathrm{C}, 61.90 ; \mathrm{H}, 9.00 ; \mathrm{N}, 6.51$. 
$\left(3 S^{*}, 5 R^{\star}\right)-N-B o c-3-a m i n o-g l y c a l(10 \mathrm{~b}):$

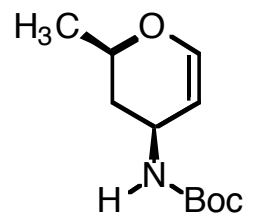

The general procedure was followed using $\mathbf{4 b}$ with modifications as for $\mathbf{4 a}$. Purification by column chromatography on silica gel (pet-ether:EtOAc $=95: 5)$ provided glycal $(\mathbf{1 0 b})$ as a white solid (yield 90\%). Mp 57-58 ${ }^{\circ} \mathrm{C}$; IR (neat film) 3341, 3065, 2979, 2934, 2876, 2139, 1681, 1647, 1527, 1391, $1234 \mathrm{~cm}^{-1} ;{ }^{1} \mathrm{H}$ NMR $\left(600 \mathrm{MHz}, \mathrm{CDCl}_{3}\right) \delta 6.38(\mathrm{~d}, J=6$ $\mathrm{Hz}, 1 \mathrm{H}), 4.58$ (d, J = $6 \mathrm{~Hz}, 1 \mathrm{H}), 4.42$ (brs, 1H), 4.36 (d, $J=7.2 \mathrm{~Hz}, 1 \mathrm{H}$ ), (qdd, $J=6,6,6$ $\mathrm{Hz}, 1 \mathrm{H}$ ), 2.25 (ddd, $J=12.6,7.2,6 \mathrm{~Hz}, 2 \mathrm{H}), 1.45(\mathrm{~s}, 9 \mathrm{H}), 1.28\left(\mathrm{~d}, J=6 \mathrm{~Hz}, 3 \mathrm{H}\right.$ ); ${ }^{13} \mathrm{C}$ NMR $\left(150 \mathrm{MHz}, \mathrm{CDCl}_{3}\right) \delta 155.64,145.97,103.03,79.64,71.81,43.74,37.74,28.61$, 21.21; HRMS (EI) Calcd. for $\mathrm{C}_{11} \mathrm{H}_{19} \mathrm{O}_{3} \mathrm{~N}\left[(\mathrm{M})^{+}\right]$213.13649, found 220.13714. Anal. Calcd. for $\mathrm{C}_{11} \mathrm{H}_{19} \mathrm{O}_{3} \mathrm{~N}$ : C, 61.95; $\mathrm{H}, 9.00 ; \mathrm{N}, 6.57$; Found: $\mathrm{C}, 61.94 ; \mathrm{H}, 9.03 ; \mathrm{N}, 6.51$.

$\left(3 R^{\star}, 5 R^{\star}\right)$-N-Acetyl-3-amino-glycal (11a):<smiles>CC1C[C@H](NC=O)C=CO1</smiles>

The general procedure was followed using 5a. Purification by column chromatography on silica gel $\left(\mathrm{CH}_{2} \mathrm{Cl}_{2}: \mathrm{CH}_{3} \mathrm{OH}=97: 3\right)$ provided glycal (11a) as a white solid (yield $75 \%$ ). Mp 130-131 ' C; IR (neat film) 3250, 3189, 3065, 2961, 2940, 2918, 2882, 1646, 1549, 1400, 1372, 1243, $1090 \mathrm{~cm}^{-1} ;{ }^{1} \mathrm{H}$ NMR (600 MHz, CDCl 3$) \delta 6.52$ (d, J = $\left.6 \mathrm{~Hz}, 1 \mathrm{H}\right), 5.59$ (brs, $1 \mathrm{H}$ ), 4.74 (dd, $J=6,5.4 \mathrm{~Hz}, 1 \mathrm{H}$ ), 4.35 (d, $6 \mathrm{~Hz}, 1 \mathrm{H}$ ), 3.87 (qdd, $J=6,6,5.4 \mathrm{~Hz}$, $1 \mathrm{H}), 1.98(\mathrm{~s}, 3 \mathrm{H}), 1.93(\mathrm{~d}, J=13.2 \mathrm{~Hz}, 1 \mathrm{H}), 1.68$ (ddd $J=13.2,6,5.4 \mathrm{~Hz}, 1 \mathrm{H}), 1.31$ (d, $J=6 \mathrm{~Hz}, 3 \mathrm{H}) ;{ }^{13} \mathrm{C}$ NMR $\left(150 \mathrm{MHz}, \mathrm{CDCl}_{3}\right) \delta 169.13,147.77,99.74,68.39,40.37$, .36.47, 23.69, 20.98; HRMS (EI) Calcd. for $\mathrm{C}_{8} \mathrm{H}_{13} \mathrm{O}_{2} \mathrm{~N}\left[(\mathrm{M})^{+}\right]$155.09463, found 155.09487. Anal. Calcd. for $\mathrm{C}_{8} \mathrm{H}_{13} \mathrm{O}_{2} \mathrm{~N}: \mathrm{C}, 61.91 ; \mathrm{H}, 8.44 ; \mathrm{N}, 9.03$; Found: $\mathrm{C}, 62.16 ; \mathrm{H}$, $8.57 ; \mathrm{N}, 9.00$. 
$\left(3 S^{\star}, 5 R^{\star}\right)$-N-Acetyl-3-amino-glycal (11b):

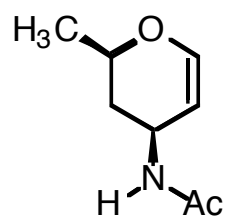

The general procedure was followed using $\mathbf{5 b}$. Purification by column chromatography on silica gel $\left(\mathrm{CH}_{2} \mathrm{Cl}_{2}: \mathrm{CH}_{3} \mathrm{OH}=97: 3\right)$ provided glycal $(11 \mathrm{~b})$ as a white solid (yield $75 \%$ ). Mp 91-93 ${ }^{\circ} \mathrm{C}$; IR (neat film) 3283, 3193, 3080, 2973, 2945, 2920, 2844, 2270, 1646, 1551, 1437, 1386, 1241, 1121, $1035 \mathrm{~cm}^{-1} ;{ }^{1} \mathrm{H}$ NMR $\left(600 \mathrm{MHz}, \mathrm{CDCl}_{3}\right) \delta 6.42$ (d, J = 6.3 $\mathrm{Hz}, 1 \mathrm{H}$ ), 5.39 (brs, $1 \mathrm{H}$ ), 4.66 (ddd, $J=6,6,6 \mathrm{~Hz}, 1 \mathrm{H}), 4.55$ (dd, $J=6.3,1.2 \mathrm{~Hz}, 1 \mathrm{H}$ ), 4.08 (qdd, $J=6,6,6,6 \mathrm{~Hz}, 1 \mathrm{H}$ ), 2.28 (ddd, $J=13.2,6,1.2 \mathrm{~Hz}, 2 \mathrm{H}$ ), 1.98 (d, $J=0.6 \mathrm{~Hz}$, $3 \mathrm{H}) 1.28(\mathrm{dd}, J=6,1.2 \mathrm{~Hz}, 3 \mathrm{H}) ;{ }^{13} \mathrm{C}$ NMR $\left(150 \mathrm{MHz}, \mathrm{CDCl}_{3}\right) \delta 169.93,146.50,102.39$, 71.77, 42.64, 37.24, 23.65, 21.15; HRMS (EI) Calcd. for $\mathrm{C}_{8} \mathrm{H}_{13} \mathrm{O}_{2} \mathrm{~N}\left[(\mathrm{M})^{+}\right]$155.09463, found 155.09518. Anal. Calcd. for $\mathrm{C}_{8} \mathrm{H}_{13} \mathrm{O}_{2} \mathrm{~N}$ : C, 61.91; $\mathrm{H}, 8.44 ; \mathrm{N}, 9.03$; Found: C, 61.96; H, 8.51; N, 8.86.

$\left(3 R^{\star}, 5 R^{\star}\right)$-N-Pivaloyl-3-amino-glycal (12a):

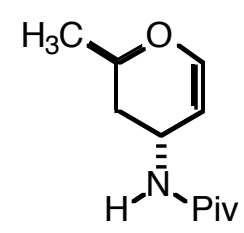

The general procedure was followed using $\mathbf{6 a}$. Purification by column chromatography on silica gel $\left(\mathrm{CH}_{2} \mathrm{Cl}_{2}: \mathrm{CH}_{3} \mathrm{OH}=98: 2\right)$ provided glycal (12a) as a white solid (yield $40 \%$ ). Mp 100-102 ${ }^{\circ} \mathrm{C}$; IR (neat film) 3311, 3158, 3065, 2969, 2922, 2873, 2364, 2296, 1630, 1524, 1461, 1399, 1240, 1202, 1089, $1048 \mathrm{~cm}^{-1} ;{ }^{1} \mathrm{H}$ NMR $\left(600 \mathrm{MHz}, \mathrm{CDCl}_{3}\right) \delta 6.53$ (d, J $=5.4 \mathrm{~Hz}, 1 \mathrm{H}$ ), 5.61 (brs, 1H), 4.75 (ddd, $J=6,5.4,1.8 \mathrm{~Hz}, 1 \mathrm{H}$ ), 4.34 (app. ddd, $J=6,6$, $1.8 \mathrm{~Hz}, 1 \mathrm{H}$ ), 3.85 (dqdd, $J=12,6.6,6,1.8 \mathrm{~Hz}, 1 \mathrm{H}$ ), 1.90 (dd, $J=14.4,1.8 \mathrm{~Hz}, 1 \mathrm{H}$ ), 1.68 (app.ddd, $J=14.4,6,2.4 \mathrm{~Hz}, 1 \mathrm{H}), 1.57(\mathrm{~s}, 9 \mathrm{H}), 1.32$ (d, J = $6.6 \mathrm{~Hz}, 3 \mathrm{H}) ;{ }^{13} \mathrm{C}$ NMR $(150$ $\left.\mathrm{MHz}, \mathrm{CDCl}_{3}\right) \delta 178.32,146.42,102.61,71.77,42.35,38.74,37.24,27.73,21.15$; HRMS (El) Calcd. for $\mathrm{C}_{11} \mathrm{H}_{19} \mathrm{O}_{2} \mathrm{~N}$ [(M) $\left.{ }^{+}\right]$197.14158, found 197.14109; Anal. Calcd. for $\mathrm{C}_{11} \mathrm{H}_{19} \mathrm{O}_{2} \mathrm{~N}$ : C, 66.97; $\mathrm{H}, 9.71 ; \mathrm{N}, 7.10$; Found: $\mathrm{C}, 67.06 ; \mathrm{H}, 9.76 ; \mathrm{N}, 6.80$. 
$\left(3 S^{*}, 5 R^{\star}\right)-N$-Pivaloyl-3-amino-glycal (12b):

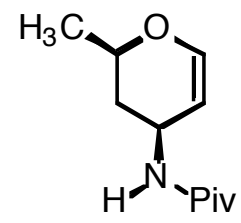

The general procedure was followed using $\mathbf{6 b}$. Purification by column chromatography on silica gel $\left(\mathrm{CH}_{2} \mathrm{Cl}_{2}: \mathrm{CH}_{3} \mathrm{OH}=98: 2\right)$ provided glycal (12b) as a white solid (yield $\left.60 \%\right)$. Mp 99-101 ' C; IR (neat film) 3314, 3126, 3069, 2960, 2930, 2869, 2360, 2341, 1627 , 1536, 1459, 1399, 1227, 1154, 1110, $1033 \mathrm{~cm}^{-1} ;{ }^{1} \mathrm{H} \mathrm{NMR}\left(600 \mathrm{MHz}, \mathrm{CDCl}_{3}\right) \delta 6.43$ (dd, $J=6.6,1.8 \mathrm{~Hz}, 1 \mathrm{H}$ ), 5.46 (brs, 1H), 4.64 (ddd, $J=7.8,6.6,1.8 \mathrm{~Hz}, 1 \mathrm{H}$ ), 4.55 (ddd, $J=$ 6.6, 4.2, $1.8 \mathrm{~Hz}, 1 \mathrm{H}$ ), 4.10 (qddd, $J=6.6,6.6,6.6,1.8 \mathrm{~Hz}, 1 \mathrm{H}$ ), 2.30 (ddd, $J=13.2,7.8$, 7.8, $1.8 \mathrm{~Hz}, 1 \mathrm{H}$ ), 2.29 (dddd, $J=13.2,6.66 .6,1.8 \mathrm{~Hz}, 1 \mathrm{H}$ ), 1.28 (d, $J=6.6 \mathrm{~Hz}, 3 \mathrm{H}$ ), 1.19 (s, 9H); ${ }^{13} \mathrm{C}$ NMR $\left(150 \mathrm{MHz}, \mathrm{CDCl}_{3}\right) \delta 178.33,146.45,102.61,71.78,42.36,38.77$, 37.26, 27.74, 21.18; HRMS (EI) Calcd. for $\mathrm{C}_{11} \mathrm{H}_{19} \mathrm{O}_{2} \mathrm{~N}\left[(\mathrm{M})^{+}\right]$197.14158, found 197.14141; Anal. Calcd. for $\mathrm{C}_{11} \mathrm{H}_{19} \mathrm{O}_{2} \mathrm{~N}$ : C, 66.97; H, 9.71; N, 7.10; Found: C, 67.14; $\mathrm{H}$, $9.79 ; \mathrm{N}, 7.10$.

$\left(3 R^{*}, 5 R^{\star}\right)-N$-Formyl-3-amino-glycal (13a):

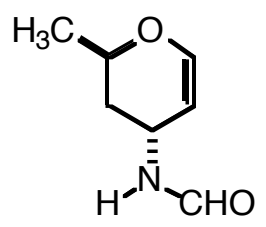

The general procedure was followed using $7 \mathbf{a}$ with modifications as for $4 \mathbf{a}$. Purification by column chromatography on silica gel $\left(\mathrm{CH}_{2} \mathrm{Cl}_{2}: \mathrm{CH}_{3} \mathrm{OH}=98: 2\right)$ provided glycal (13a) as a white solid (yield 85\%). Mp 60-62 ${ }^{\circ} \mathrm{C}$; IR (neat film) 3287, 3039, 2965, 2925, 2876 , 2859, 2118, 1666, 1530, 1461, 1384 1260, $1093 \mathrm{~cm}^{-1} ;{ }^{1} \mathrm{H}$ NMR $\left(600 \mathrm{MHz}, \mathrm{CDCl}_{3}\right) \delta$ $8.11(\mathrm{~s}, 1 \mathrm{H}), 6.54(\mathrm{~d}, J=9 \mathrm{~Hz}, 1 \mathrm{H}), 5.63$ (brs, 1H), 4.75 (ddd, J = 9, 7.8, $3 \mathrm{~Hz}, 1 \mathrm{H}$ ), 3.89, (dqd, $J=18,9.6,2.4 \mathrm{~Hz}, 1 \mathrm{H}$ ), 1.97 (ddd, $J=21,3,2.4 \mathrm{~Hz}, 1 \mathrm{H}$ ), 1.73 (ddd, $J=21$, $18,7.8 \mathrm{~Hz}, 1 \mathrm{H}), 1.33$ (d, $J=9.6 \mathrm{~Hz}, 3 \mathrm{H}) ;{ }^{13} \mathrm{C} \mathrm{NMR}\left(150 \mathrm{MHz}, \mathrm{CDCl}_{3}\right) \delta 160.22,148.14$, 99.23, 68.31, 39.29, 36.38, 20.95; HRMS (El) Calcd. for $\mathrm{C}_{7} \mathrm{H}_{11} \mathrm{O}_{2} \mathrm{~N}\left[(\mathrm{M})^{+}\right]$141.07898, found 141.07838 . 
$\left(3 S^{\star}, 5 R^{\star}\right)-N$-Formyl-3-amino-glycal (13b)

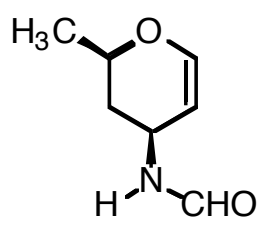

The general procedure was followed using $\mathbf{7 b}$ with modifications as for $\mathbf{4 a}$. Purification by column chromatography on silica gel $\left(\mathrm{CH}_{2} \mathrm{Cl}_{2}: \mathrm{CH}_{3} \mathrm{OH}=98: 2\right)$ provided glycal (13b) as a white solid (yield 90\%). Mp 76-78 ${ }^{\circ} \mathrm{C}$; IR (neat film) 3287, 3039, 2965, 2925, 2876, 2859, 2118, 1666, 1530, 1461, $13841260,1093 \mathrm{~cm}^{-1} ;{ }^{1} \mathrm{H}$ NMR $\left(600 \mathrm{MHz}, \mathrm{CDCl}_{3}\right) \delta$ 8.16 (brs, 1H), 6.44 (dd, J = 9, $3 \mathrm{~Hz}, 1 \mathrm{H}$ ), 5.55 (brs, 1H), 4.77 (ddd, J = 9.2, 7.8, $3 \mathrm{~Hz}$, 1H), 4.56, (ddd, $J=9,5.4,2.4 \mathrm{~Hz}, 1 \mathrm{H}$ ), 4.09 (qddd, $J=9.6,9.2,7.8,2.4 \mathrm{~Hz}, 1 \mathrm{H}$ ), 2.29 (ddd, $J=17.4,7.8,2.4 \mathrm{~Hz}, 1 \mathrm{H}$ ), 1.60 (ddd, $J=17.4,9.2,2.4 \mathrm{~Hz}, 1 \mathrm{H}$ ), 1.29 (d, J = 9.6 $\mathrm{Hz}, 3 \mathrm{H}) ;{ }^{13} \mathrm{C}$ NMR $\left(150 \mathrm{MHz}, \mathrm{CDCl}_{3}\right) \delta 161.00,146.80,101.80,71.61,41.36,37.08$, 21.12; HRMS (EI) Calcd. for $\mathrm{C}_{7} \mathrm{H}_{11} \mathrm{O}_{2} \mathrm{~N}\left[(\mathrm{M})^{+}\right]$141.07898, found 141.07916; Anal. Calcd. for $\mathrm{C}_{7} \mathrm{H}_{11} \mathrm{O}_{2} \mathrm{~N}$ : C, 59.56; H, 7.85; N, 9.92; Found: C, 59.87; H, 7.92; N, 9.62.

$\left(3 R^{*}, 5 R^{*}\right)-N-F o r m y l-N-m e t h y l-3-a m i n o-g l y c a l ~(14 a)$ :

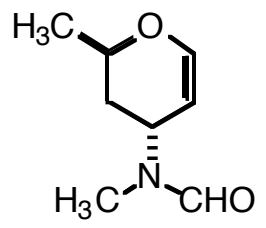

The general procedure was followed using $\mathbf{8 a}$. Purification by column chromatography on silica gel $\left(\mathrm{CH}_{2} \mathrm{Cl}_{2}: \mathrm{CH}_{3} \mathrm{OH}=98: 2\right)$ provided glycal (14a) as a white solid (yield $80 \%$ ). Mp 128-131 ' C; IR (neat film) 3583, 3335, 3059, 2974, 2928, 2872, 2116, 1676, 1642 , 1444, 1385, $12361089,1032 \mathrm{~cm}^{-1} ;{ }^{1} \mathrm{H}$ NMR $\left(600 \mathrm{MHz}, \mathrm{CDCl}_{3}\right) \delta 8.35$ (s, 0.75H), 8.11 (s, 0.25H), $6.63(\mathrm{dd}, J=6.6,1.2 \mathrm{~Hz}, 0.75 \mathrm{H}), 6.61(\mathrm{~d}, J=1.2 \mathrm{~Hz}, 0.25 \mathrm{H}), 4.91-4.87(\mathrm{~m}$, $0.25 \mathrm{H}$ ), 4.67 (ddd, $J=6.6,6,1.8 \mathrm{~Hz}, 0.75 \mathrm{H}$ ), 4.58 (ddd, $J=4.8,4.8,1.2 \mathrm{~Hz}, 0.25 \mathrm{H}$ ), 4.07 (dqd, $J=12,6,6 \mathrm{~Hz}, 0.25 \mathrm{H}), 4.02-3.96(\mathrm{~m}, 0.75 \mathrm{H}), 4.02-3.96(\mathrm{~m}, 0.75 \mathrm{H}), 3.94-$ $3.91(\mathrm{~m}, 0.75 \mathrm{H}), 2.94(\mathrm{~s}, 0.75 \mathrm{H}), 2.88(\mathrm{~s}, 2.25 \mathrm{H}), 1.98-1.88(\mathrm{~m}, 1 \mathrm{H}), 1.83$ (ddd, $J=16$, 10, $6 \mathrm{~Hz}, 0.1 \mathrm{H}$ ), 1.73 (ddd, $J=16,12,6 \mathrm{~Hz}, 0.9 \mathrm{H}$ ), 1.32 (d, $J=6 \mathrm{~Hz}, 2.25 \mathrm{H}$ ), 1.30 (s, $0.75 \mathrm{H}) ;{ }^{13} \mathrm{C}$ NMR $\left(150 \mathrm{MHz}, \mathrm{CDCl}_{3}\right) \delta 163.01,148.50,97.68,68.03,49.77,35.15$, 29.14, 21.60; HRMS (EI) Calcd. for $\mathrm{C}_{8} \mathrm{H}_{13} \mathrm{O}_{2} \mathrm{~N}\left[(\mathrm{M})^{+}\right]$155.09463, found 155.09504 . 
$\left(3 S^{*}, 5 R^{\star}\right)-N$-Formyl-N-methyl-3-amino-glycal (14b):

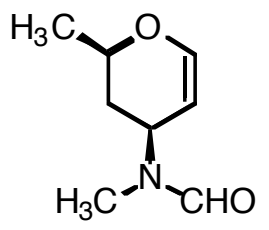

The general procedure was followed using $\mathbf{8 b}$. Purification by column chromatography on silica gel $\left(\mathrm{CH}_{2} \mathrm{Cl}_{2}: \mathrm{CH}_{3} \mathrm{OH}=98: 2\right)$ provided glycal $(14 \mathrm{~b})$ as an oil (yield $\left.84 \%\right)$. IR (neat film) 3583, 3335, 3059, 2974, 2928, 2872, 2116, 1676, 1642, 1444, 1385, 1236 1089, $1032 \mathrm{~cm}^{-1} ;{ }^{1} \mathrm{H}$ NMR (600 MHz, $\left.\mathrm{CDCl}_{3}\right) \delta 8.15(\mathrm{~s}, 0.7 \mathrm{H}), 8.10(\mathrm{~s}, 0.3 \mathrm{H}), 6.55(\mathrm{~d}, J=$ $6 \mathrm{~Hz}, 1 \mathrm{H}$ ), 5.22 (ddd, $J=8.4,8.4,1.8 \mathrm{~Hz}, 0.3 \mathrm{H}$ ), 4.51 (d, $J=6 \mathrm{~Hz}, 0.7 \mathrm{H}), 4.47$ (d, $J=$ $6.6 \mathrm{~Hz}, 0.3 \mathrm{H}$ ), 4.35 (ddd, $J=8.4,8.4,1.8 \mathrm{~Hz}, 0.7 \mathrm{H}$ ), 4.13 (qdd, $J=6,6,6 \mathrm{~Hz}, 1 \mathrm{H}$ ), 2.83 $(\mathrm{s}, 0.9 \mathrm{H}), 2.78(\mathrm{~s}, 2.1 \mathrm{H}), 1.93-1.78(\mathrm{~m}, 1.4 \mathrm{H}), 1.69-1.60(\mathrm{~m}, 0.6 \mathrm{H}), 1.06(\mathrm{~d}, J=6 \mathrm{~Hz}$, $0.9 \mathrm{H}), 1.02(\mathrm{~d}, J=6 \mathrm{~Hz}, 2.1 \mathrm{H}) ;{ }^{13} \mathrm{C}$ NMR $\left(150 \mathrm{MHz}, \mathrm{CDCl}_{3}\right) \delta$ 162.61, 148.25, 101.08, 71.80, 51.60, 34.93, 29.80, 21.25; HRMS (EI) Calcd. for $\mathrm{C}_{8} \mathrm{H}_{13} \mathrm{O}_{2} \mathrm{~N}\left[(\mathrm{M})^{+}\right]$155.09463, found 155.09504 .

$\left(3 R^{*}, 5 R^{*}\right)-N, N$-Diboc-3-amino-glycal (15a):

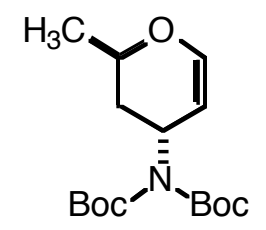

The general procedure was followed using $9 \mathrm{a}(0.260 \mathrm{~g}, 0.83 \mathrm{mmol}), \mathrm{W}(\mathrm{CO})_{6},(0.044 \mathrm{~g}$, $0.12 \mathrm{mmol})$, DABCO $(0.186 \mathrm{~g}, 1.7 \mathrm{mmol})$, THF $(3 \mathrm{~mL})$. Purification by column chromatography on silica gel (pet-ether:EtOAc $=95: 5)$ provided glycal $(\mathbf{1 5 a})$ as an oil (0.052 g, yield 20\%). IR (neat film) 3062, 2961, 2853, 1745, 1707, 1674, 1459, 1368, 1339, 1260, 1241, 1150, $1113 \mathrm{~cm}^{-1} ;{ }^{1} \mathrm{H}$ NMR $\left(400 \mathrm{MHz}, \mathrm{CDCl}_{3}\right) \delta 6.51$ (dd, $J=6.4,1.6$ $\mathrm{Hz}, 0.9 \mathrm{H}$ ), 6.39 (dd, $J=6.4,1.6 \mathrm{~Hz}, 0.1 \mathrm{H}$ ), 5.35 (ddd, $J=5.6,2.4,2 \mathrm{~Hz}, 0.9 \mathrm{H}$ ), 4.99$4.91(\mathrm{~m}, 0.1 \mathrm{H}), 4.78-4.65(\mathrm{~m}, 0.9 \mathrm{H}), 4.37(\mathrm{dq}, J=9,3.6 \mathrm{~Hz}, 0.1 \mathrm{H}), 4.26(\mathrm{dd}, J=2.4,2.4$ $\mathrm{Hz}, 0.5 \mathrm{H}), 3.88(\mathrm{dd}, J=1.6,1.6 \mathrm{~Hz}, 0.5 \mathrm{H}), 2.41(\mathrm{ddd}, J=12.6,9,5.6 \mathrm{~Hz}, 0.5 \mathrm{H}), 2.12-$ $2.03(\mathrm{~m}, 0.5 \mathrm{H}), 1.94(\mathrm{ddd}, J=12.6,9,5.6 \mathrm{~Hz}, 0.5 \mathrm{H}), 1.79-1.64(\mathrm{~m}, 0.5 \mathrm{H}), 1.51(\mathrm{~d}, J=$ 2.8, 9H), $1.49(\mathrm{~s}, \mathrm{~Hz}, 9 \mathrm{H}), 1.32-1.24(\mathrm{~m}, 3 \mathrm{H}) ;{ }^{13} \mathrm{C}$ NMR $\left(100 \mathrm{MHz}, \mathrm{CDCl}_{3}\right) \delta 152.73$, 147.17, 98.11, 83.11, 68.57, 46.57, 37.17, 28.24, 21.85; HRMS (FAB ${ }^{+}$Calcd. for $\mathrm{C}_{16} \mathrm{H}_{27} \mathrm{O}_{5} \mathrm{NLi}\left[(\mathrm{MLi})^{+}\right]$320.2049, found 320.2039. 
$\left(3 S^{*}, 5 R^{\star}\right)-N, N-$ Diboc-3-amino-glycal (15b):<smiles>CC1CC(N(C(=O)OC(C)(C)C)C(=O)OC(C)(C)C)C=CO1</smiles>

The general procedure was followed with modifications as for 9a. Purification by column chromatography on silica gel (pet-ether:EtOAc $=95: 5)$ provided glycal $(\mathbf{1 5 b})$ as a white solid (yield $85 \%$ ). Mp 34-36 ${ }^{\circ} \mathrm{C}$; IR (neat film) 3134, 3067, 2982, 2940, 2883, 2296, 1739, 1703, 1647, 1480, 1459, 1397, 1372, 1347, 1237,1116, $1035 \mathrm{~cm}^{-1} ;{ }^{1} \mathrm{H}$ NMR $\left(400 \mathrm{MHz}, \mathrm{CDCl}_{3}\right) \delta 6.39$ (dd, $\left.J=6.4,2.4 \mathrm{~Hz}, 1 \mathrm{H}\right), 4.95$ (dddd, $J=10.4,6.4,2.4,2$ $\mathrm{Hz}, 1 \mathrm{H}$ ), 4.60 (ddd, $J=6.4,2.4,2 \mathrm{~Hz}, 1 \mathrm{H}$ ), 4.08 (dqd, $J=10.4,6,2 \mathrm{~Hz}, 1 \mathrm{H}$ ), 2.16 (app.ddd, $J=12.4,10.4,10 \mathrm{~Hz}, 1 \mathrm{H}$ ), 1.95 (dddd, $J=12.4,6.4,2.4,2 \mathrm{~Hz}, 1 \mathrm{H}$ ), 1.5 (s, $18 \mathrm{H}), 1.29$ (d, $J=6 \mathrm{~Hz}, 3 \mathrm{H}) ;{ }^{13} \mathrm{C}$ NMR $\left(100 \mathrm{MHz}, \mathrm{CDCl}_{3}\right) \delta 152.92,145.39,102.62$,

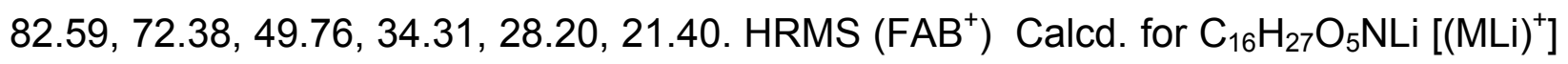
320.2049, found 320.2035. Anal. Calcd. for $\mathrm{C}_{16} \mathrm{H}_{27} \mathrm{O}_{5} \mathrm{~N}$ : C, 61.32; $\mathrm{H}, 8.68 ; \mathrm{N}, 4.47$; Found: $\mathrm{C}, 61.34 ; \mathrm{H}, 8.68 ; \mathrm{N}, 4.47$.

(3S, 5R)-5-(tert-Butyldimethylsilanyloxy)-1-(trimethylsilyl)hex-1-yn-3-ol (2b):

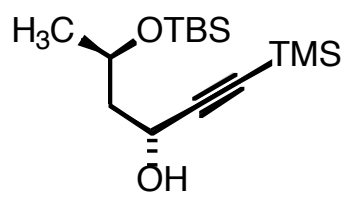

$(R, R)-\mathbf{2 b}$

A $50 \mathrm{~mL}$ Schlenk flask was charged with $\mathrm{Zn}(\mathrm{OTf})_{2}(1.810 \mathrm{~g}, 5 \mathrm{mmol})$. Vacuum $(\leq 0.5$ mbar) was applied and the flask was heated to $125^{\circ} \mathrm{C}$ overnight. The flask was cooled to room temperature, the vacuum was released, and (+)- $N$-methylephedrine $(0.936 \mathrm{~g}$, $5.2 \mathrm{mmol}$ ) was added. Vacuum ( $\leq 0.5 \mathrm{mbar}$ ) was applied for $0.5 \mathrm{~h}$ and then released. To the flask was added toluene $(12 \mathrm{~mL})$ and $\mathrm{Et}_{3} \mathrm{~N}(0.73 \mathrm{~mL}, 5.2 \mathrm{mmol})$ The resulting mixture was stirred for $2 \mathrm{~h}$ at room temperature before trimethylsilyacetylene $(0.73 \mathrm{~mL}$, $5.2 \mathrm{mmol}$ ) was added neat. After stirring for $30 \mathrm{~min}$, aldehyde $(R)-1^{1}(0.404 \mathrm{~g}, 2 \mathrm{mmol})$ in toluene $(2 \mathrm{~mL})$ was added over a $2 \mathrm{~h}$ period. The reaction mixture was stirred for 18

\footnotetext{
${ }^{1}$ Ohta, K.; Miyagaw, O.; Tsutsui, H.; Mitsunobu, O. Bull. Chem. Soc. Jpn. 1993, 66, 523.
} 
$h$ at which time it was quenched by the addition of a saturated solution of $\mathrm{NH}_{4} \mathrm{Cl}(40$ $\mathrm{mL}$ ). The organic phase was separated and the aqueous layer was extracted with ether $(3 \times 25 \mathrm{~mL})$. The organic layers were combined, dried over $\mathrm{Na}_{2} \mathrm{SO}_{4}$, and evaporated under reduced pressure. The residual oil was purified by column chromatography on silica gel (pet-ether:EtOAc=98:2) to yield compound $\mathbf{2 b}$ as a clear oil $(0.6 \mathrm{~g}, 75 \%$ yield). $[\alpha]^{26}{ }_{D}-43.01\left(c=1.0, \mathrm{CHCl}_{3}\right)$.

(3S, 5R)-5-(tert-Butyldimethylsilanyloxy)-1-(trimethylsilyl)-3-aminohex-1-yne (3b):

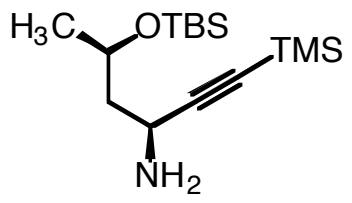

3b

Prepared as described earlier in the racemic series from $\mathbf{2 b} .[\alpha]^{26}{ }_{D}-23.94(c=1.38$, $\left.\mathrm{CHCl}_{3}\right)$.

(3S, 5R)-3-amino-hex-1-yn-5-ol, $N$-(tert-Butoxycarbonyl) derivative (4b):

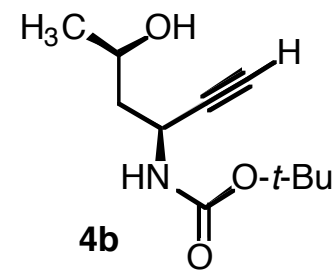

Prepared as described earlier for racemate. $[\alpha]^{26}{ }_{D}-50.2\left(c=1.2, \mathrm{CHCl}_{3}\right)$.

(3S, 5R)-N-Boc-3-amino-glycal (10b):

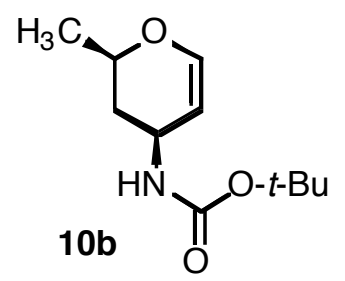

Prepared as described earlier for racemate. $[\alpha]^{26}{ }_{D}-40.43\left(c=1.3, \mathrm{CHCl}_{3}\right)$. 
(3S, 5R)-N,N-Dimethyl-3-amino-glycal (16):

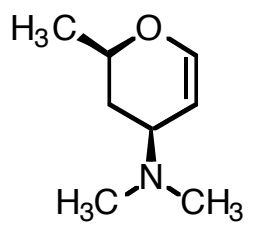

16

To a stirred solution of $10 \mathrm{~b}(1.00 \mathrm{~g}, 4.7 \mathrm{mmol})$ in DMF $(10 \mathrm{~mL})$ at room temperature was carefully added $\mathrm{NaH}(0.281 \mathrm{~g}, 11.7 \mathrm{mmol})$. The reaction mixture was stirred for $15 \mathrm{~min}$, at which time $\mathrm{CH}_{3} \mathrm{l}(4.3 \mathrm{~mL}, 28.2 \mathrm{mmol})$ was added dropwise. The reaction was stirred for $6 \mathrm{~h}$ and quenched with $\mathrm{H}_{2} \mathrm{O}(40 \mathrm{~mL})$. The organic layer was separated, the aqueous layer was extracted with EtOAc $(3 \times 25 \mathrm{~mL})$, and the combined organic layers were washed with $\mathrm{NaHCO}_{3}$, dried over $\mathrm{Na}_{2} \mathrm{SO}_{4}$, and evaporated under reduced pressure. The residual oil was purified by column chromatography on silica gel (pet-ether:EtOAc $=$ $8: 2)$ to yield the $N$-methyl derivative of $10 \mathrm{~b}$ as a clear oil $(0.950 \mathrm{~g}, 89 \%$ yield $)$. $[\alpha]^{26}{ }_{D}$ -39.2 (c = 0.75, $\mathrm{CHCl}_{3}$ ); IR (neat film) 3360, 3061, 2975, 2928, 2874, 1693, 1644, 1383, 1238, $1141 \mathrm{~cm}^{-1} ;{ }^{1} \mathrm{H}$ NMR $\left(600 \mathrm{MHz}, \mathrm{CDCl}_{3}\right) \delta 6.47(\mathrm{~d}, J=5.7 \mathrm{~Hz}, 1 \mathrm{H}), 4.98$ (brs, $\left.0.6 \mathrm{H}\right)$, 4.78 (brs, 0.4H), 4.50 (d, J =5.7 Hz, 1H), 4.13-4.05 (m, 1H), 2.69 (brs, 3H), 1.94-1.82 $(\mathrm{m}, 1 \mathrm{H}), 1.70-1.54(\mathrm{~m}, 1 \mathrm{H}), 1.47(\mathrm{~s}, 18 \mathrm{H}), 1.32-1.23(\mathrm{~m}, 3 \mathrm{H}) ;{ }^{13} \mathrm{C}$ NMR $(150 \mathrm{MHz}$, $\left.\mathrm{CDCl}_{3}\right) \delta 156.10,147.00,102.72,79.69,72.01,47.95,33.32,28.65,21.37$; HRMS (EI) Calcd. for $\mathrm{C}_{12} \mathrm{H}_{21} \mathrm{O}_{3} \mathrm{~N}\left[(\mathrm{M})^{+}\right]$227.15214, found 227.15110. Anal. Calcd. for $\mathrm{C}_{12} \mathrm{H}_{21} \mathrm{O}_{3} \mathrm{~N}$ : C, $63.41 ; \mathrm{H}, 9.31 ; \mathrm{N}, 6.16$; Found: $\mathrm{C}, 63.28 ; \mathrm{H}, 9.38 ; \mathrm{N}, 6.07$. To a stirred solution the $\mathrm{N}$-methyl derivative of $10 \mathrm{~b}(0.420 \mathrm{~g}, 1.8 \mathrm{mmol})$ in THF $(14 \mathrm{~mL})$ was added LAH (1M in THF, $0.200 \mathrm{~g}, 5.5 \mathrm{~mL}, 5.5 \mathrm{mmol}$ ) at $0{ }^{\circ} \mathrm{C}$ dropwise. The solution was allowed to come to room temperature as it was stirred for $18 \mathrm{~h}$. The reaction was carefully quenched stepwise with $\mathrm{H}_{2} \mathrm{O}(0.2 \mathrm{~mL}), \mathrm{NaOH}(15 \%$ soln, $0.4 \mathrm{~mL})$, then $\mathrm{H}_{2} \mathrm{O}(0.6 \mathrm{~mL})$ at $0{ }^{0} \mathrm{C}$, after which it was stirred $30 \mathrm{~min}$ at room temperature. The reaction mixture was dried over $\mathrm{Na}_{2} \mathrm{SO}_{4}$, filtered through Celite, and evaporated under reduced pressure. The residual oil was purified by column chromatography on silica gel (pet-ether:EtOAc $=90: 10$ ) to yield 16 as a pure oil $(0.2414 \mathrm{~g}, 95 \%$ yield $) .[\alpha]^{26}{ }_{D}-29.8\left(c=0.8, \mathrm{CHCl}_{3}\right)$; IR (neat film) $3369,3060,2973,2936,2873,2834,2787,1644,1460,1384,1238,1049 \mathrm{~cm}^{-1} ;{ }^{1} \mathrm{H}$ NMR $\left(600 \mathrm{MHz}, \mathrm{CDCl}_{3}\right) \delta 6.41(\mathrm{dd}, J=6.6,1.8 \mathrm{~Hz}, 1 \mathrm{H}), 4.63(\mathrm{dd}, J=6.6,1.2 \mathrm{~Hz}, 1 \mathrm{H})$, 4.01 (dqd, $J=12.6,6.6,1.8 \mathrm{~Hz} .1 \mathrm{H}$ ), 3.36 (dddd, $J=10.8,6.6,1.8,1.2 \mathrm{~Hz} .1 \mathrm{H}$ ), 2.26 (s, 
$6 \mathrm{H}$ ), 1.82 (ddd, $J=12.6,6.6,1.8 \mathrm{~Hz}, 1 \mathrm{H}$ ), 1.57 (ddd, $J=12.6,12.6,10.8 \mathrm{~Hz}, 1 \mathrm{H}$ ), 1.29 $(\mathrm{d}, J=6.6 \mathrm{~Hz}, 3 \mathrm{H}) ;{ }^{13} \mathrm{C} \mathrm{NMR}\left(150 \mathrm{MHz}, \mathrm{CDCl}_{3}\right) \delta 147.23,100.04,71.81,57.18,40.13$, 30.72, 21.45; HRMS (EI) Calcd. for $\mathrm{C}_{8} \mathrm{H}_{15} \mathrm{ON}\left[(\mathrm{M})^{+}\right]$141.11536, found 141.11505 .

\section{1,2-di-O-acetyl-D-desosamine (18):}

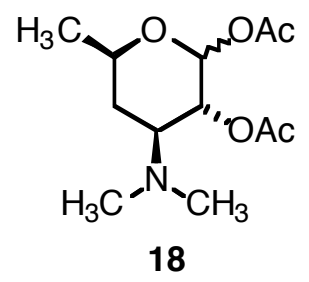

To a stirred solution of 16 (30 mg, $0.22 \mathrm{mmol})$ in $t-\mathrm{BuOH}: \mathrm{H}_{2} \mathrm{O}=1: 1(1 \mathrm{~mL})$ at room temperature was added $\left(\mathrm{CH}_{3}\right)_{3} \mathrm{NO}-\mathrm{H}_{2} \mathrm{O}(36 \mathrm{mg}, 0.33 \mathrm{mmol})$ and citric acid $(82 \mathrm{mg}, 0.44$ mmol). A solution of $\mathrm{OsO}_{4}(6 \mathrm{mg}, 0.02 \mathrm{mmol})$ in $\mathrm{CH}_{3} \mathrm{CN}(0.5 \mathrm{~mL})$ was added dropwise and the reaction mixture was stirred at room temperature for $18 \mathrm{~h}$. The reaction was azeotropically dried with benzene $(3 \times 0.5 \mathrm{~mL})$ and taken up in $\mathrm{CH}_{2} \mathrm{Cl}_{2}(5 \mathrm{~mL})$. To the reaction mixture cooled to $0{ }^{\circ} \mathrm{C}$ was added $\mathrm{Et}_{3} \mathrm{~N}(0.81 \mathrm{~mL}, 1.3 \mathrm{mmol})$, DMAP $(15 \mathrm{mg}$, $0.11 \mathrm{mmol})$, and acetic anhydride $(0.12 \mathrm{~mL}, 1.3 \mathrm{mmol})$ dropwise. The reaction was allowed to come to room temperature over $12 \mathrm{~h}$ with stirring. The reaction was quenched with $\mathrm{NaHCO}_{3}$, the organic phase was separated, and the aqueous phase was extracted with $\mathrm{CH}_{2} \mathrm{Cl}_{2}(6 \times 5 \mathrm{~mL})$. The organic layers were combined, dried over $\mathrm{Na}_{2} \mathrm{SO}_{4}$, and evaporated under reduced pressure. The residual oil was purified by column chromatography on silica gel (pet-ether:EtOAc:EtOH $=1: 1: 1$ ) to yield 1,2diacetyl- $\beta$-D-desosamine (18) as a clear oil (18 $\mathrm{mg}, 32 \%$ yield $) .[\alpha]^{26}{ }_{\mathrm{D}}-32.4(c=0.3$, $\mathrm{CHCl}_{3}$ ); IR (neat film) 3431, 2974, 2919, 2849, 2786, 1744, 1643, 1456, 1443, 1372, 1239, 1219, 1162, 1084, $10401003 \mathrm{~cm}^{-1} ;{ }^{1} \mathrm{H}$ NMR $\left(600 \mathrm{MHz}, \mathrm{CDCl}_{3}\right) \delta 5.60$ (d, J= 7.8 $\mathrm{Hz}, 1 \mathrm{H}$ ), 4.94 (dd, $J=10.2,7.8 \mathrm{~Hz}, 1 \mathrm{H}$ ), 3.72 (dqd, $J=12,6.6,1.8 \mathrm{~Hz} .1 \mathrm{H}), 2.80$ (ddd, $J$ $=12,10.2,4.2, \mathrm{~Hz} .1 \mathrm{H}$ ), $2.28(\mathrm{~s}, 6 \mathrm{H}), 2.09(\mathrm{~s}, 3 \mathrm{H}), 2.06(\mathrm{~s}, 3 \mathrm{H}), 1.80$ (ddd, $J=13.2,4.2$, $1.8 \mathrm{~Hz}, 1 \mathrm{H}$ ), 1.40 (ddd, $J=13.2,12,12 \mathrm{~Hz}, 1 \mathrm{H}), 1.29$ (d, $J=6.6 \mathrm{~Hz}, 3 \mathrm{H}) ;{ }^{13} \mathrm{C}$ NMR $(150$ $\left.\mathrm{MHz}, \mathrm{CDCl}_{3}\right) \delta 170.18,169.82,93.89,70.72,69.94,63.34,40.85,30.68,29.91,21.33$, 21.22; HRMS (EI) Calcd. for $\mathrm{C}_{12} \mathrm{H}_{21} \mathrm{O}_{5} \mathrm{~N}\left[(\mathrm{M})^{+}\right]$259.14197, found 259.14167. $[\alpha]^{26}$ $-32.4\left(c=0.3, \mathrm{CHCl}_{3}\right)$. Further elution (pet-ether:EtOAc:EtOH = 1:1:1) provided 1,2diacetyl- $\alpha$-D-desosamine (18) as a clear oil (8 mg, $14 \%$ yield). IR (neat film) 3431 , 
2974, 2919, 2849, 2786, 1744, 1643, 1456, 1443, 1372, 1239, 1219, 1162, 1084, 1040 $1003 \mathrm{~cm}^{-1} ;{ }^{1} \mathrm{H}$ NMR $\left(600 \mathrm{MHz}, \mathrm{CDCl}_{3}\right) \delta 6.24(\mathrm{~d}, J=4.2 \mathrm{~Hz}, 1 \mathrm{H}), 5.04(\mathrm{dd}, J=10.8,4.2$ $\mathrm{Hz}, 1 \mathrm{H}$ ), 4.05 (dqd, $J=12,6,2.4 \mathrm{~Hz} .1 \mathrm{H}$ ), 3.15 (ddd, $J=12,10.8,3.6, \mathrm{~Hz} .1 \mathrm{H}$ ), ), 2.31 (s, 6H), $2.15(\mathrm{~s}, 3 \mathrm{H}), 2.06(\mathrm{~s}, 3 \mathrm{H}), 1.86(\mathrm{ddd}, J=12.6,3.6,2.4 \mathrm{~Hz}, 1 \mathrm{H}), 1.43$ (ddd, $J=$ 12.6, $12,12 \mathrm{~Hz}, 1 \mathrm{H}), 1.22$ (d, $J=6 \mathrm{~Hz}, 3 \mathrm{H}) ;{ }^{13} \mathrm{C} \mathrm{NMR}\left(150 \mathrm{MHz}, \mathrm{CDCl}_{3}\right) \delta 170.52$, 169.82, 90.97, 69.10, 67.51, 57.89, 40.63, 3174, 29.91, 21.33, 21.22. 


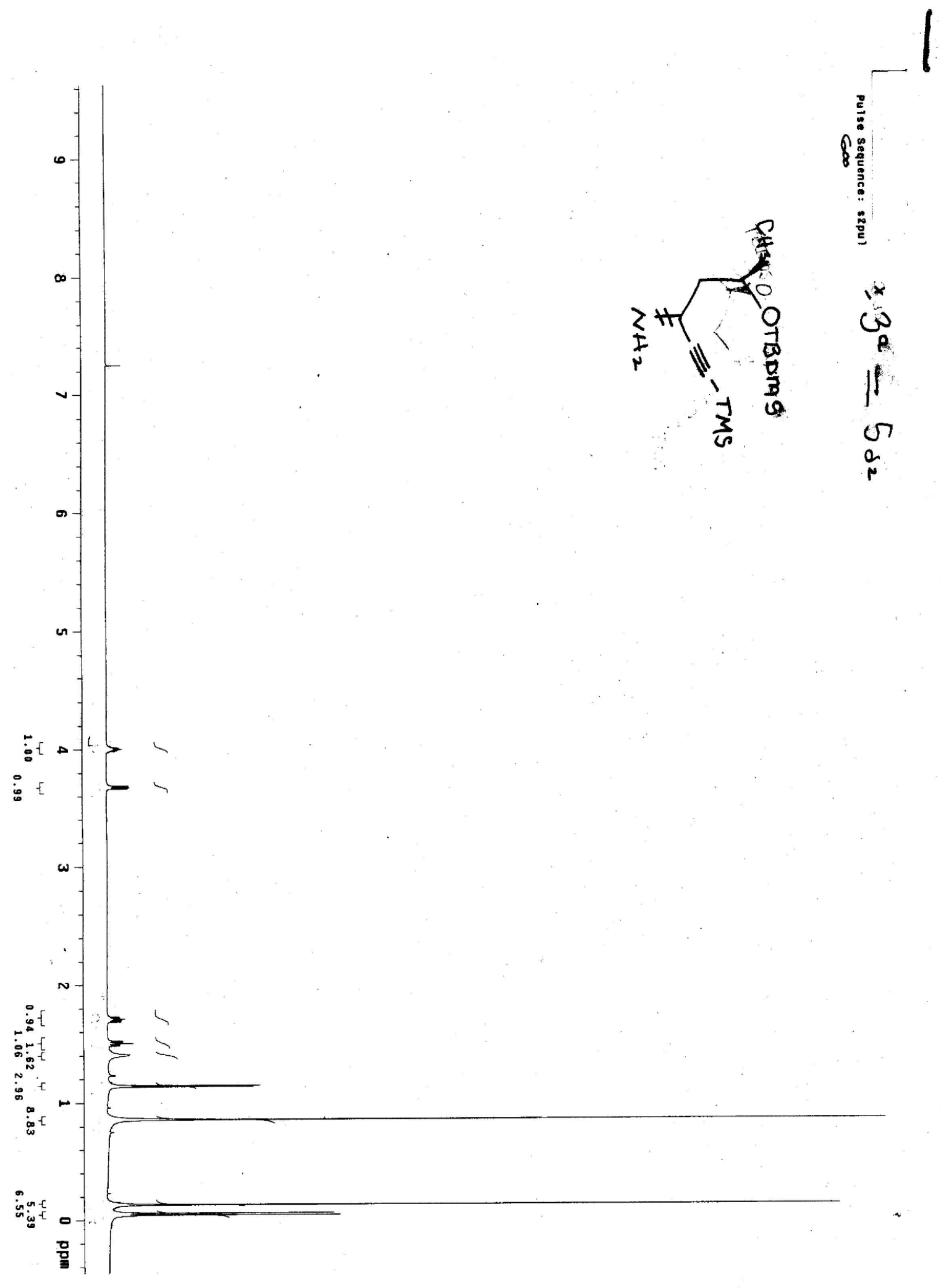




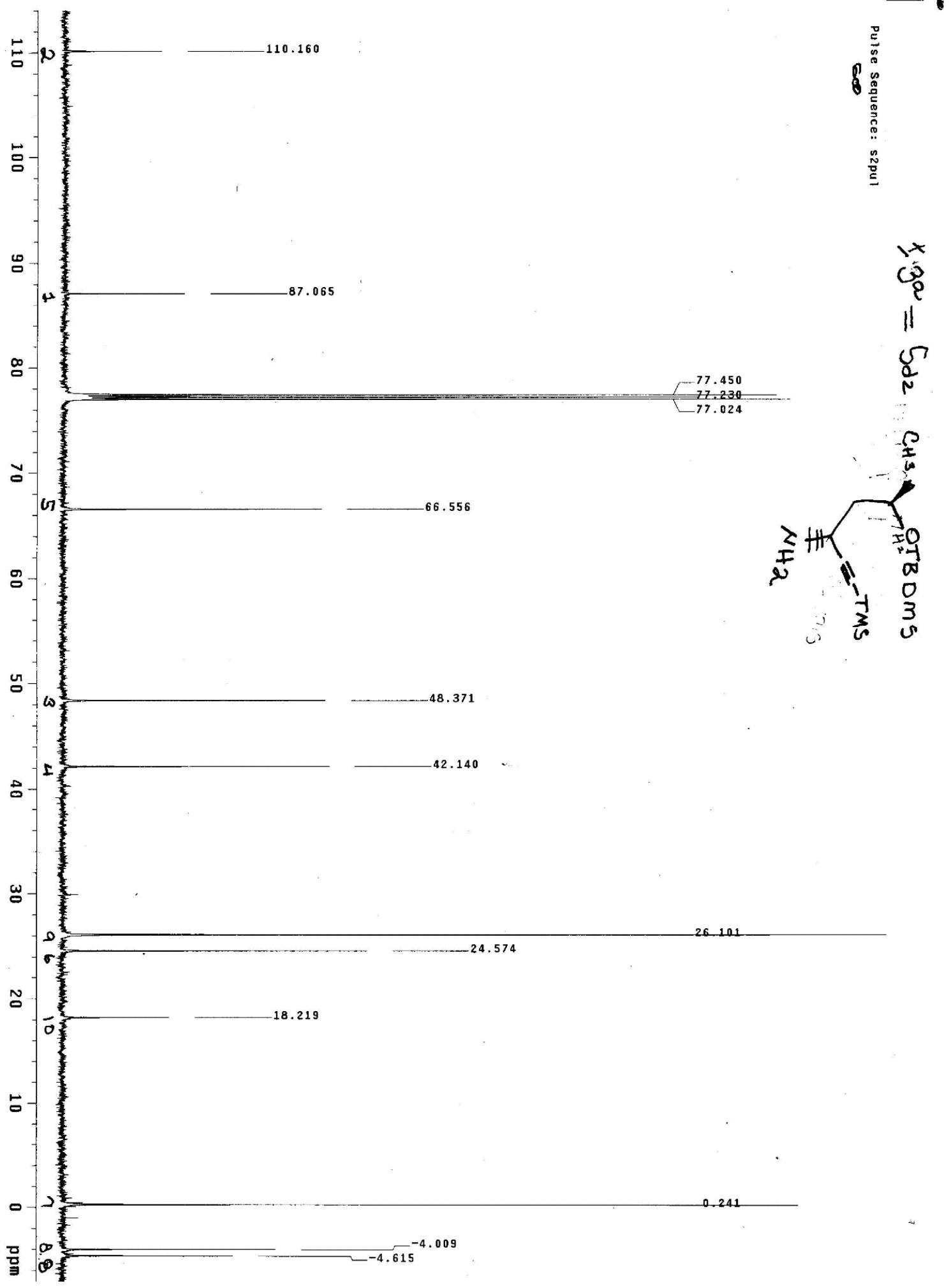




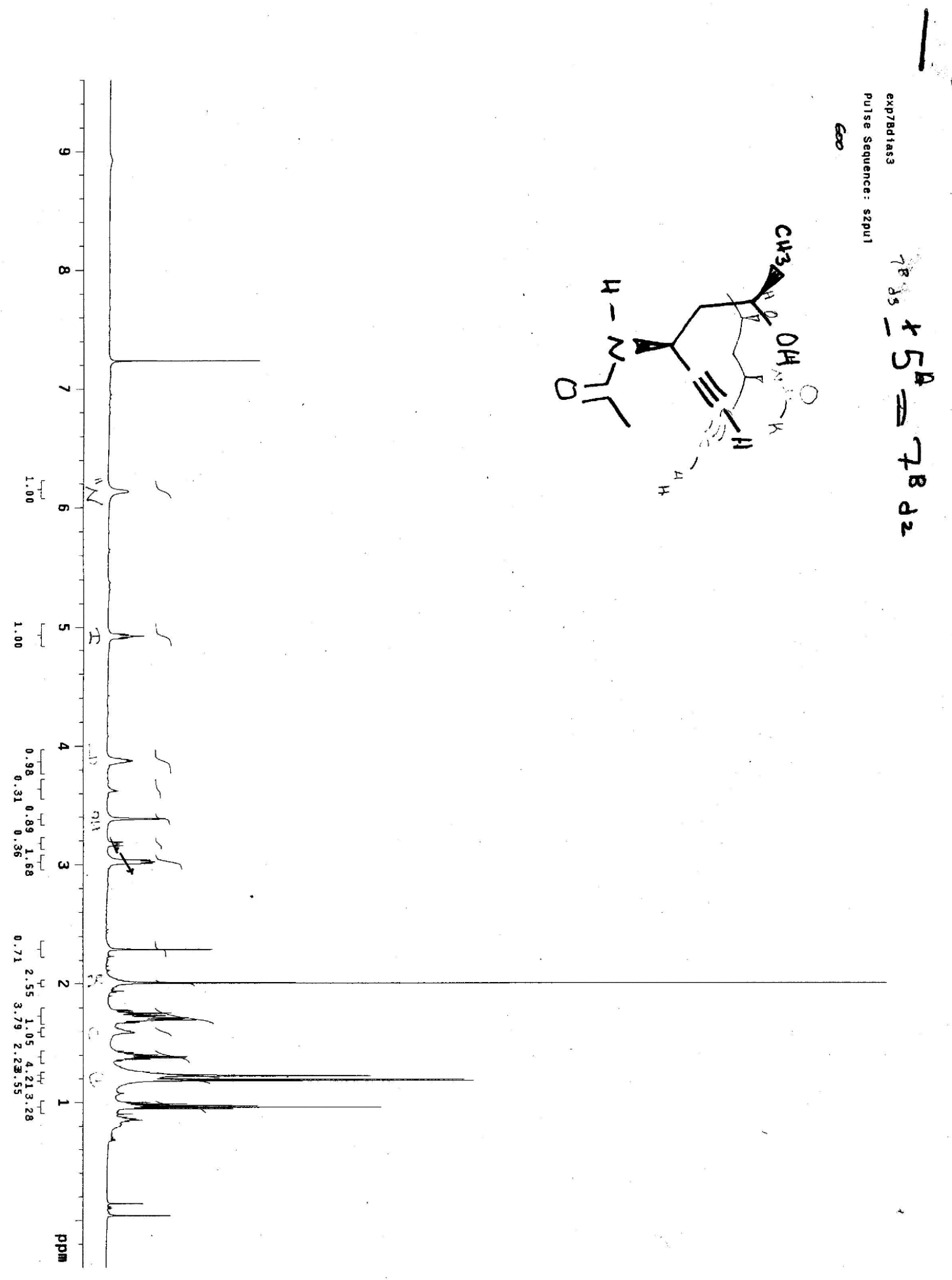




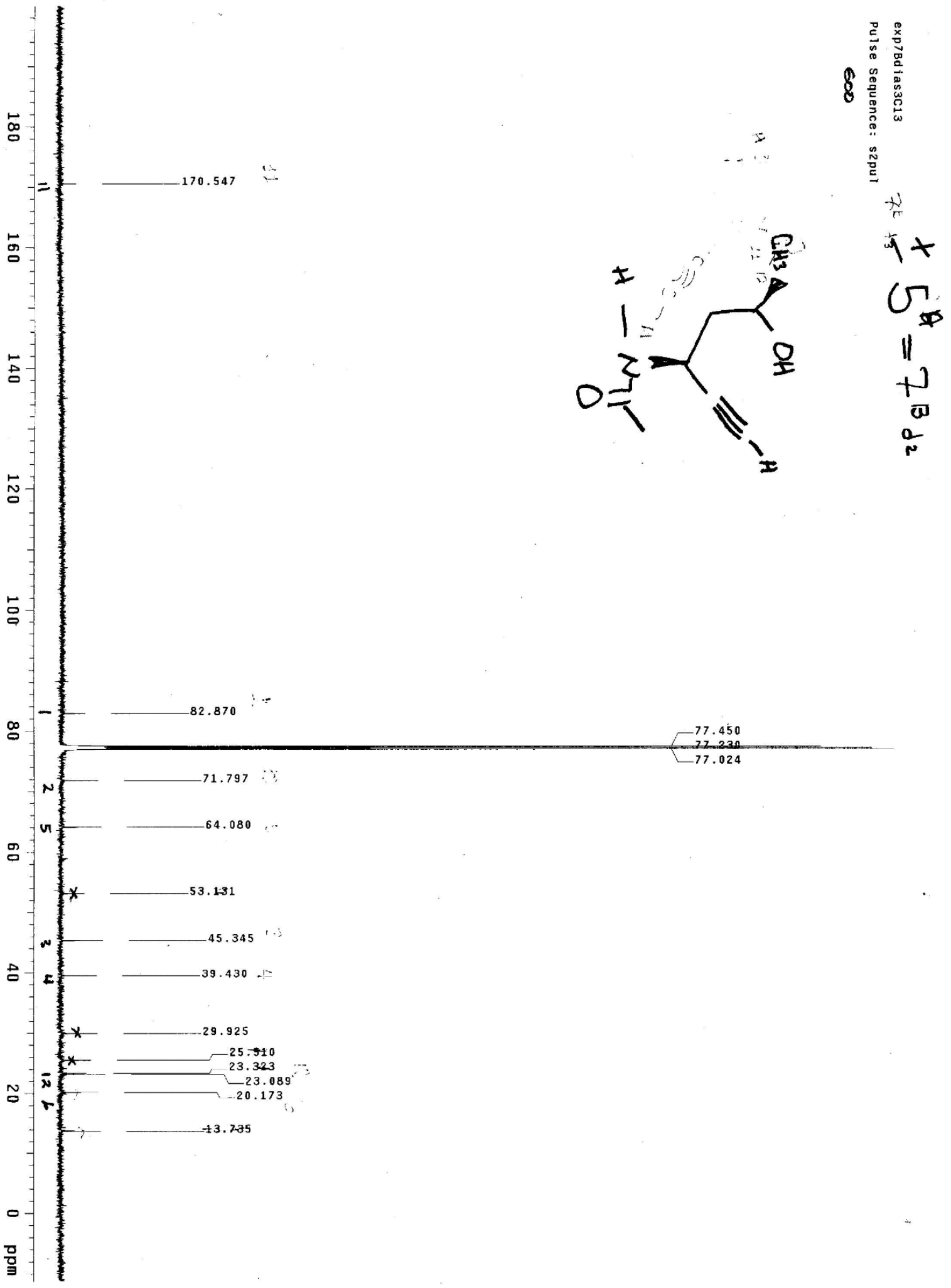




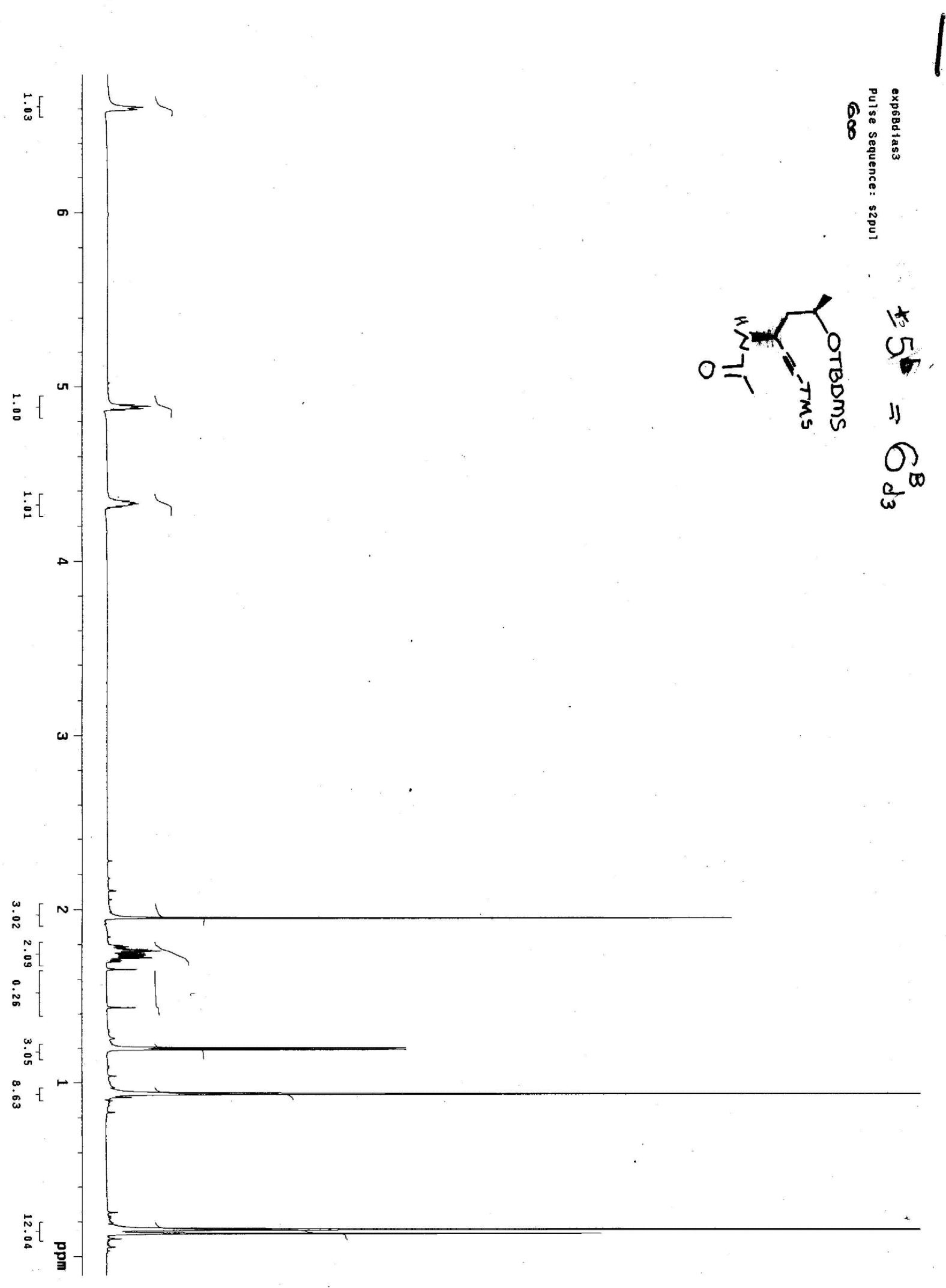



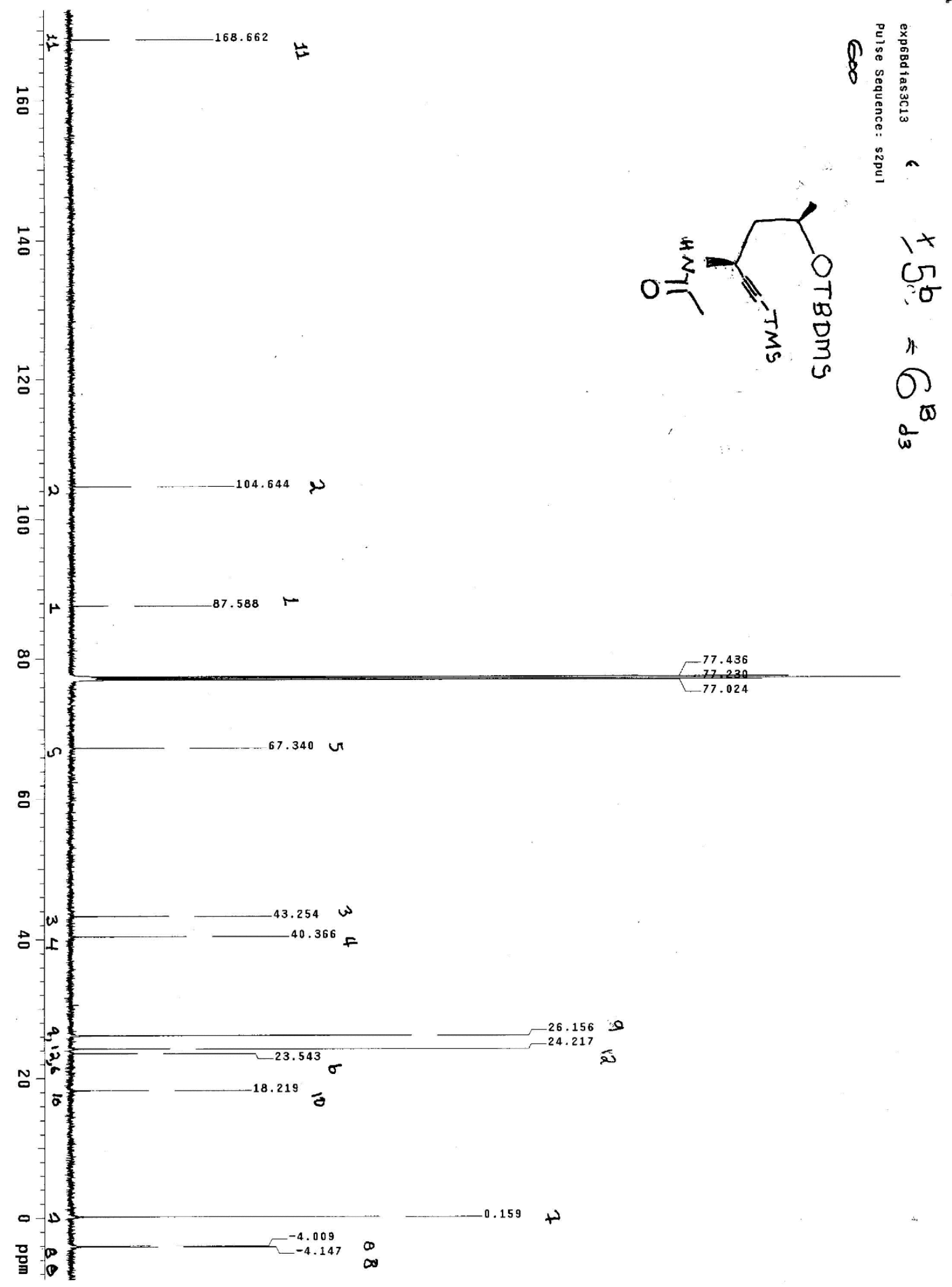


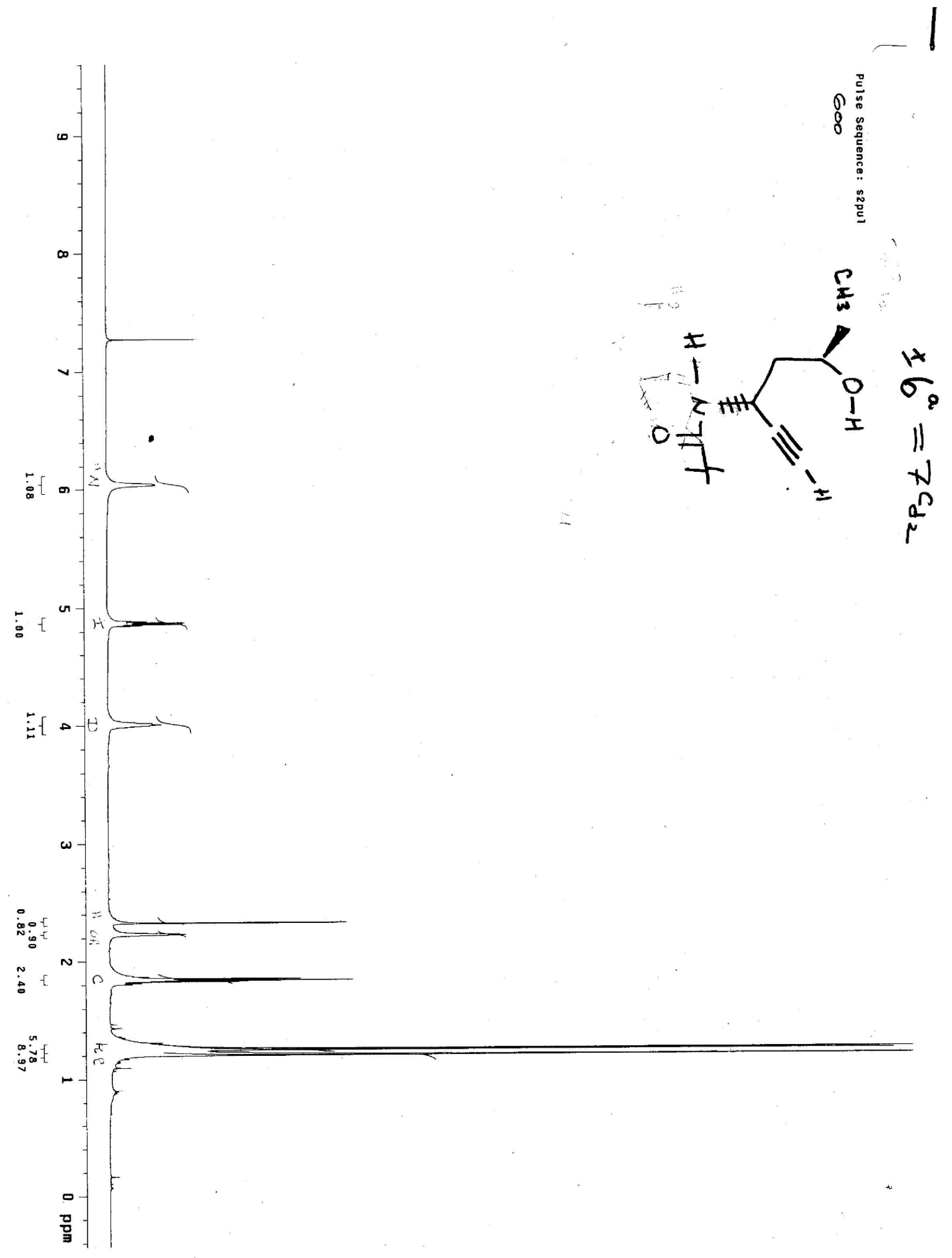




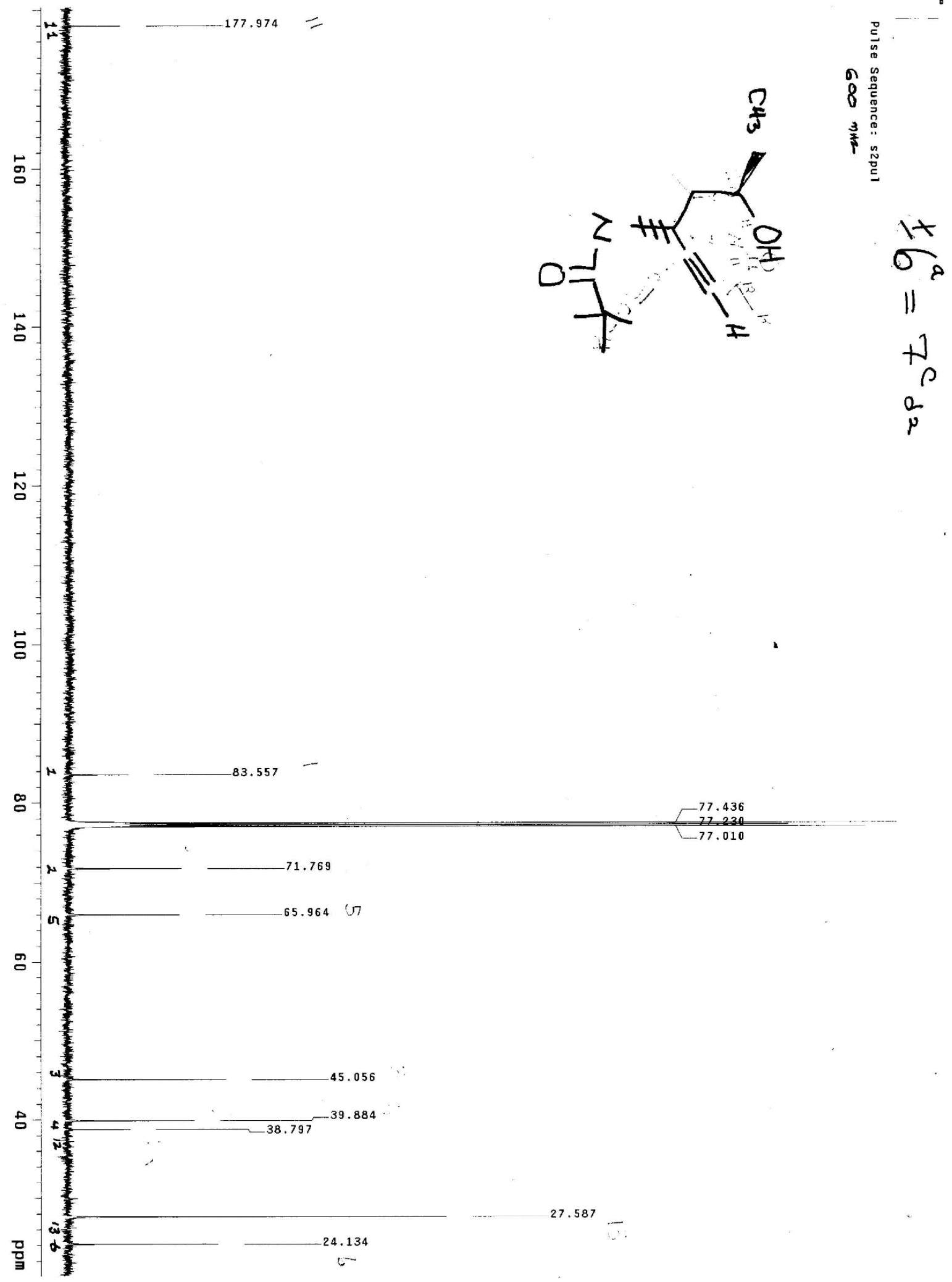




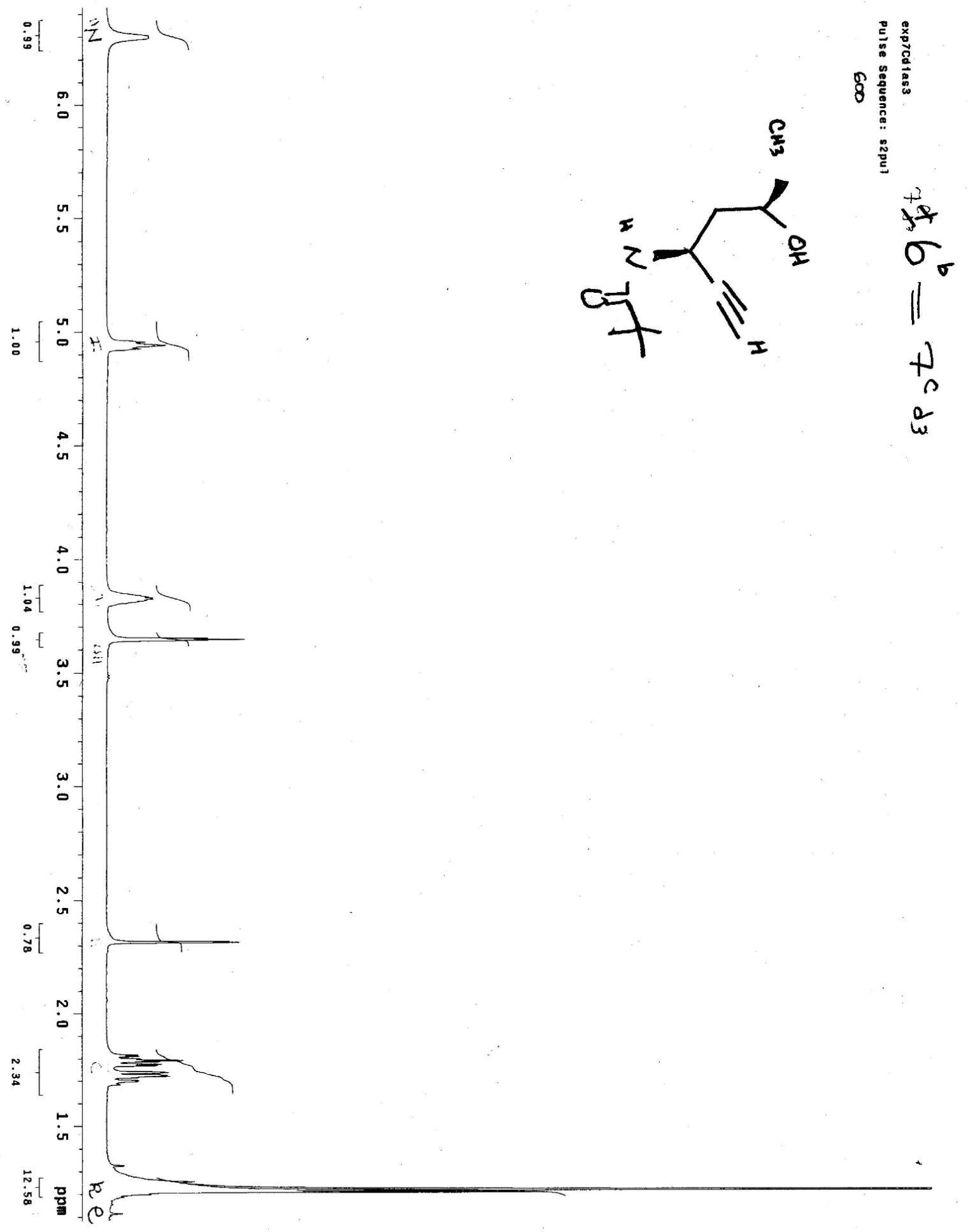




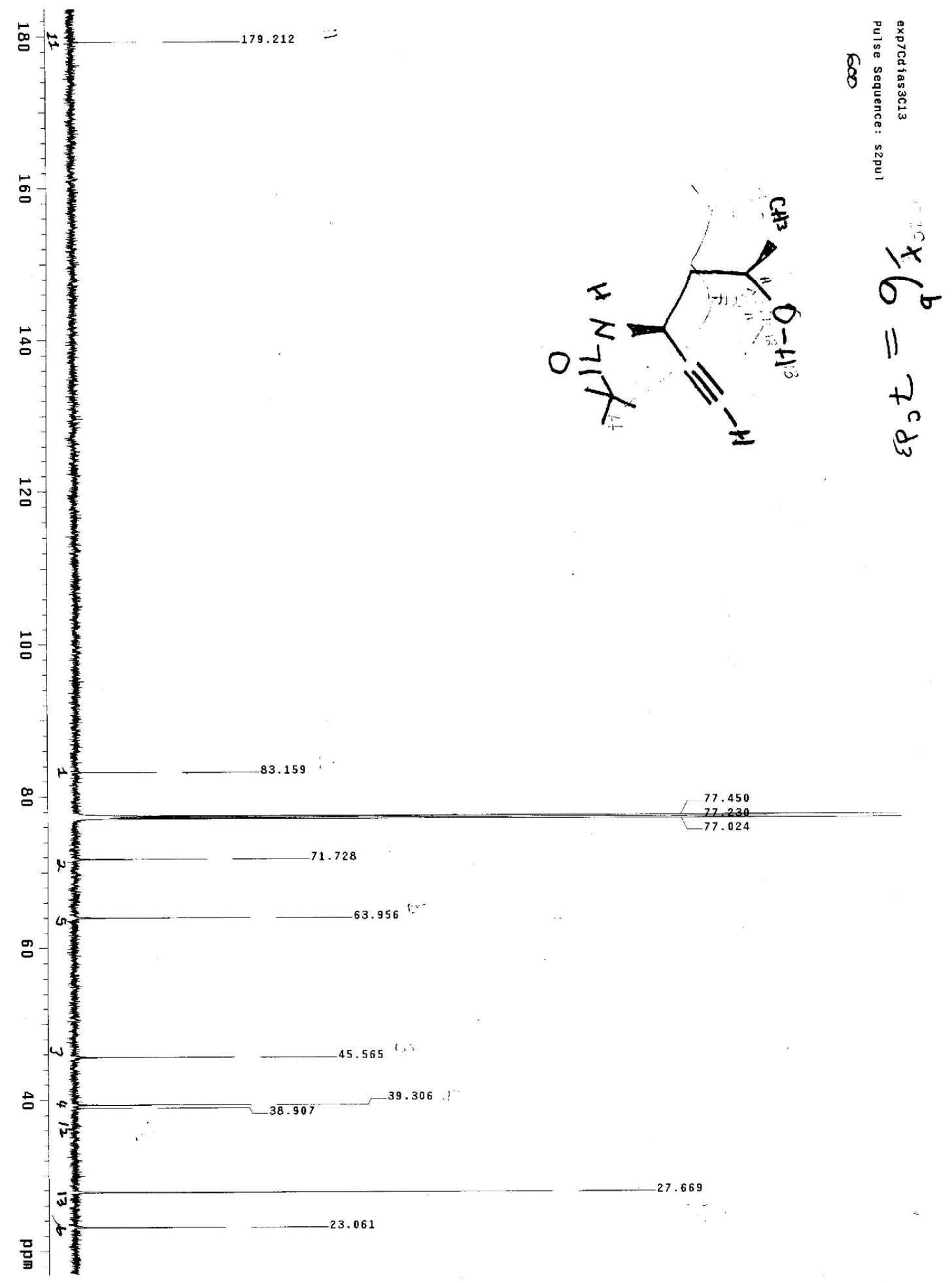




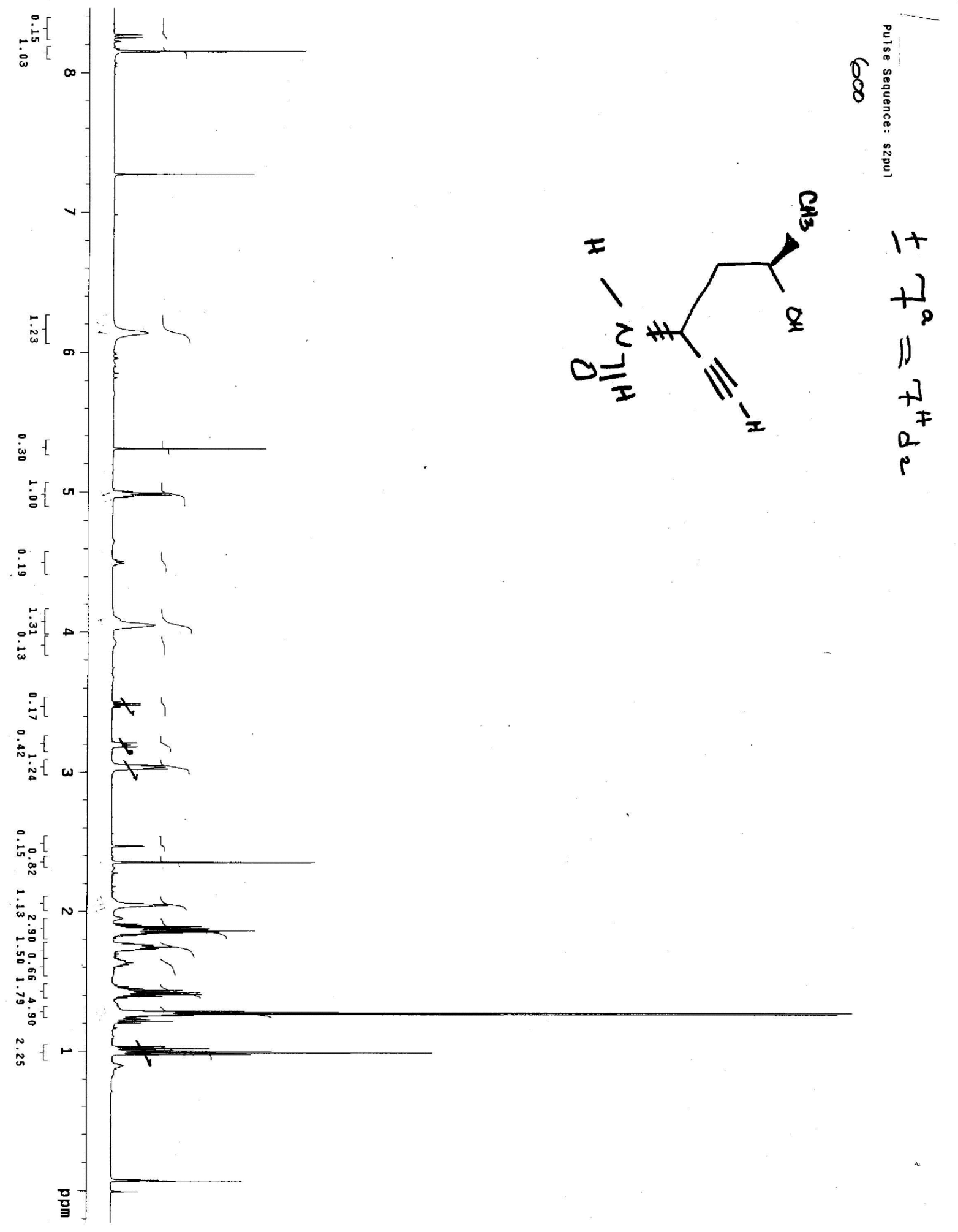




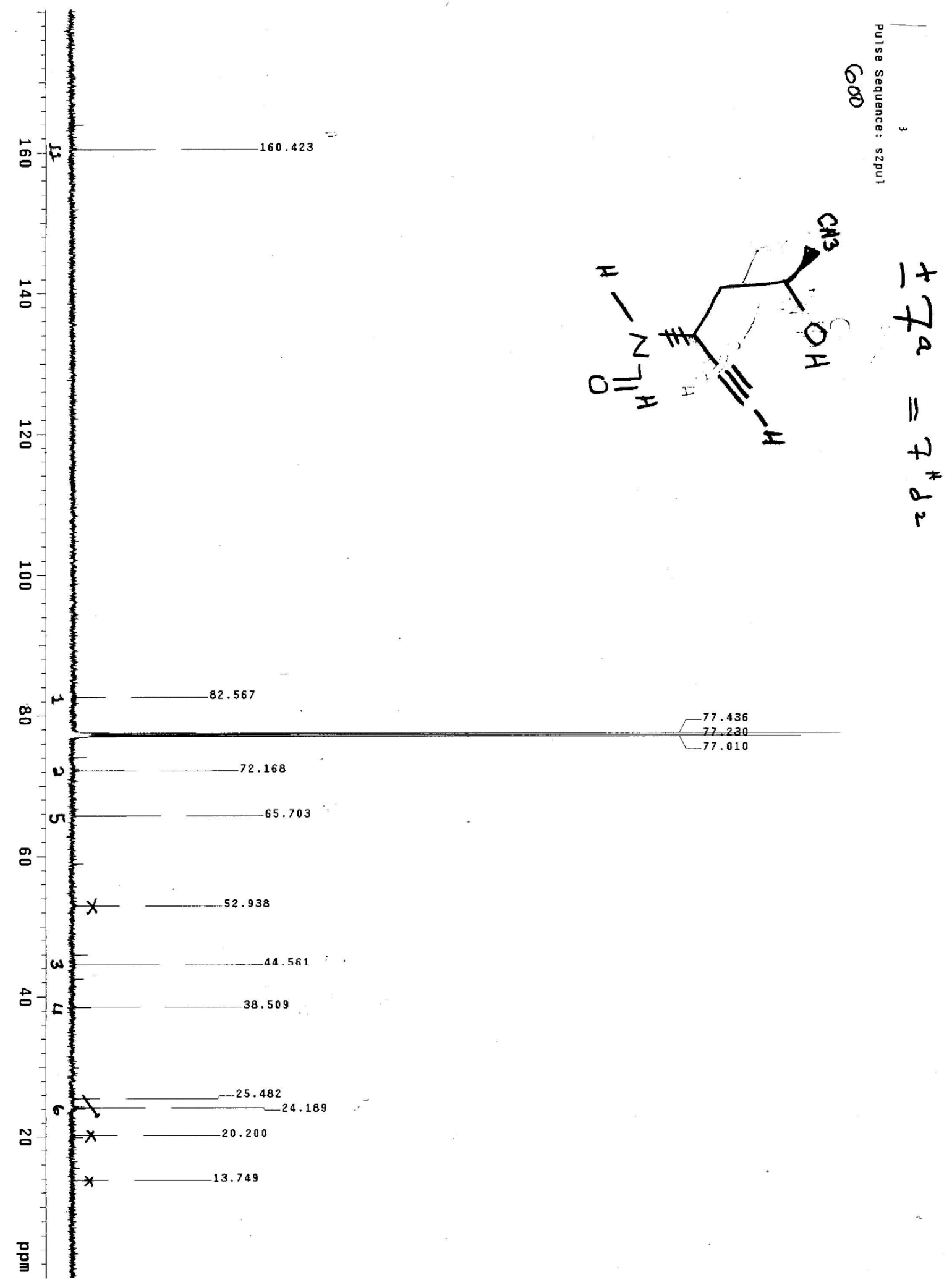



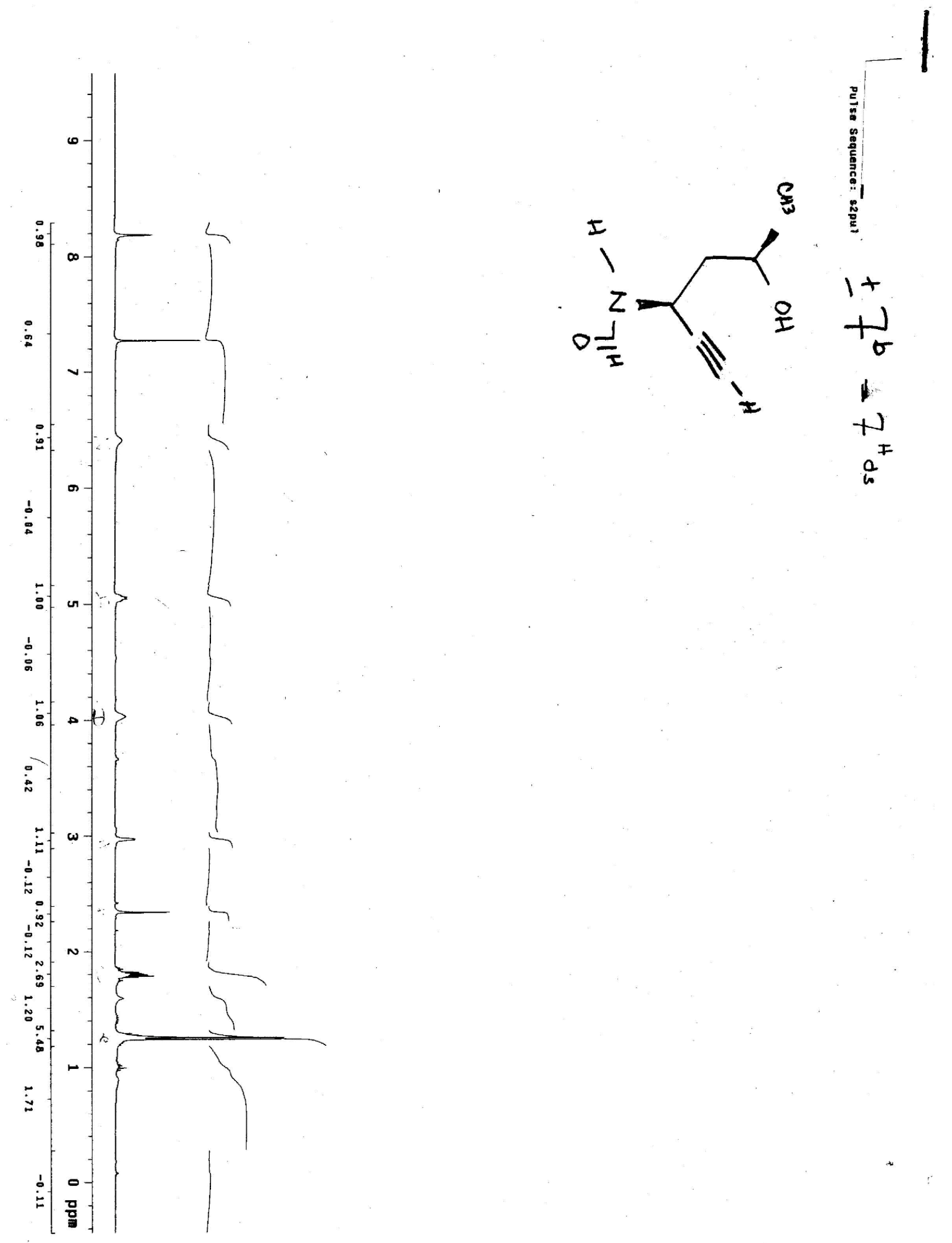


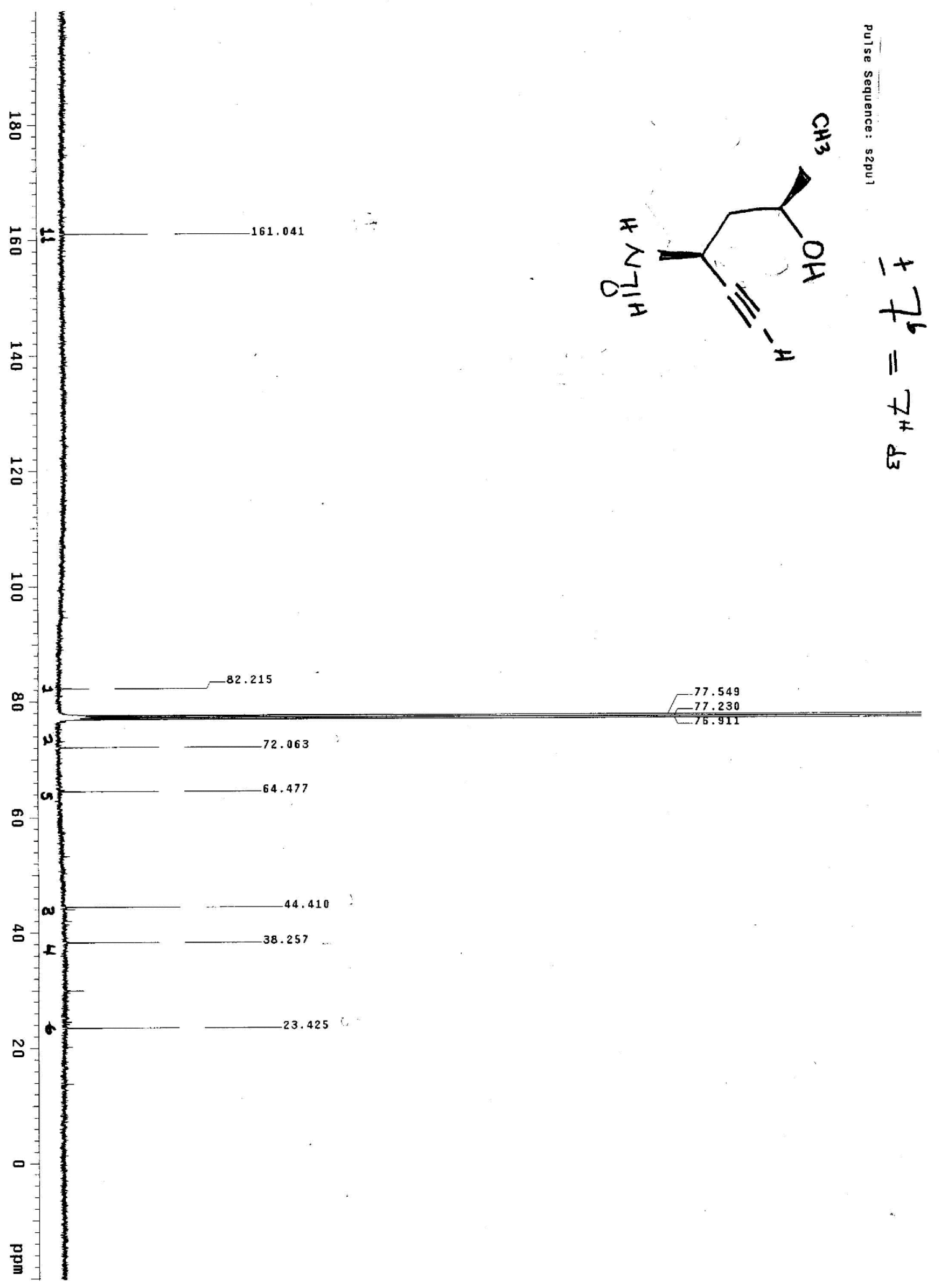




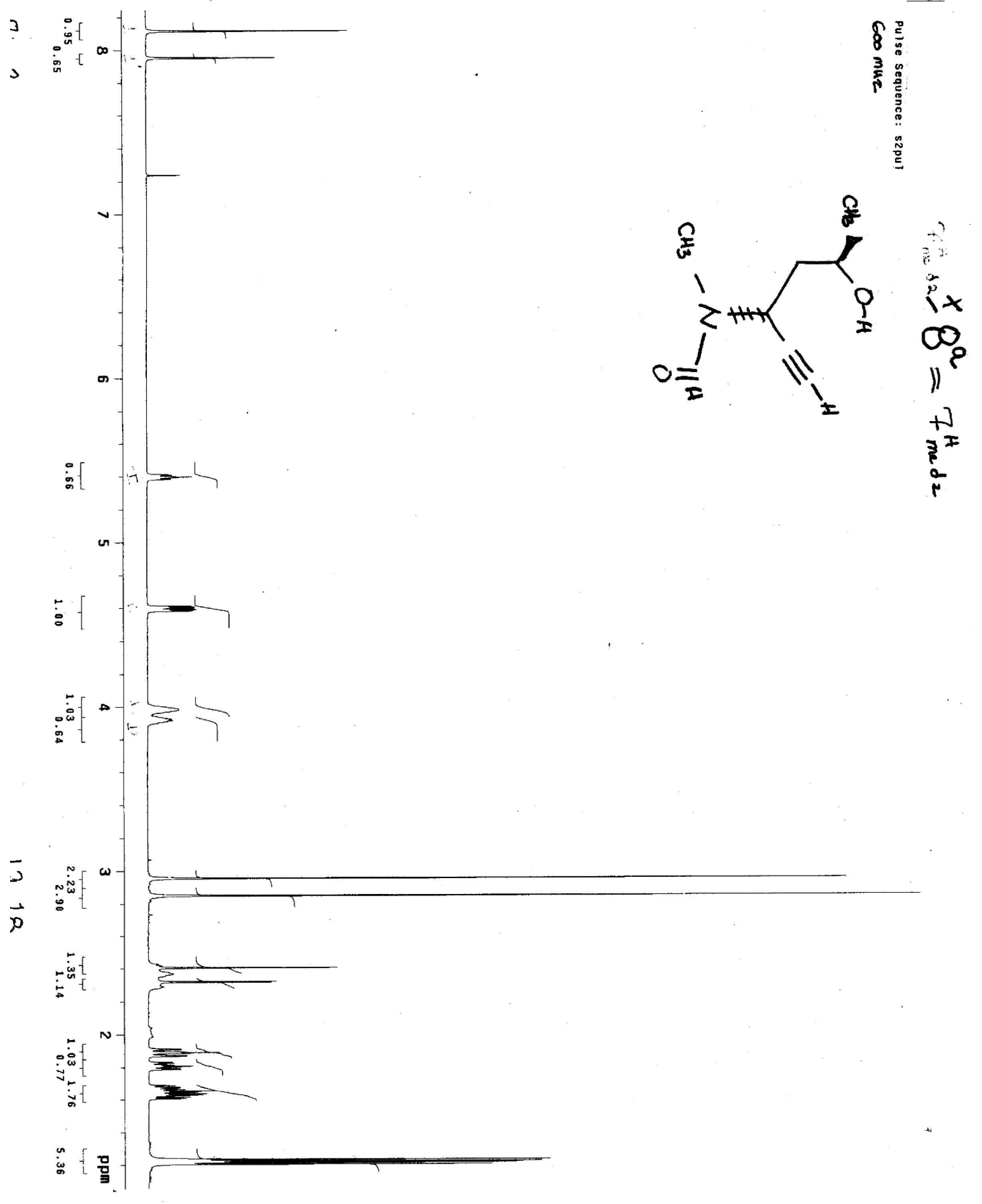




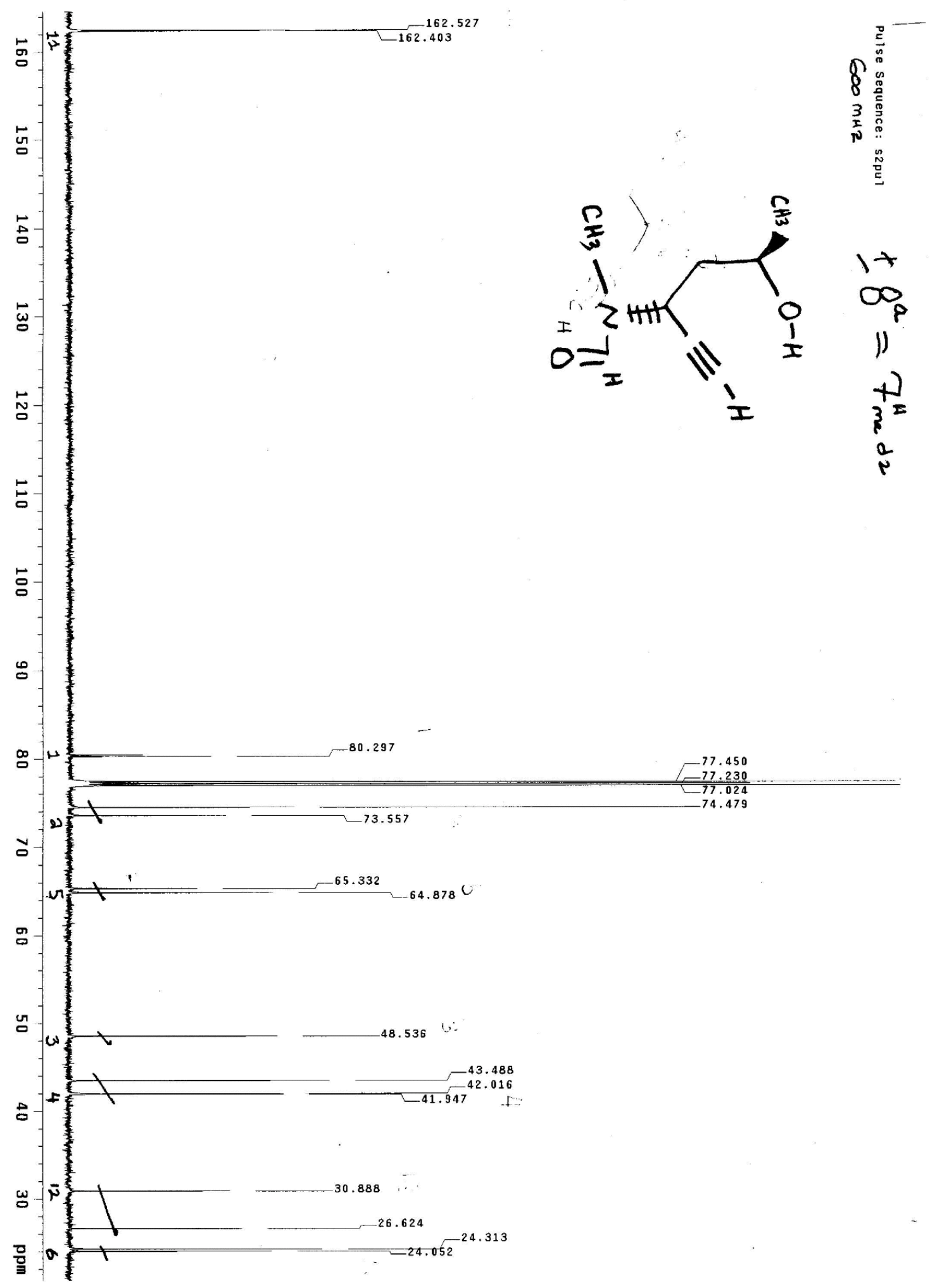




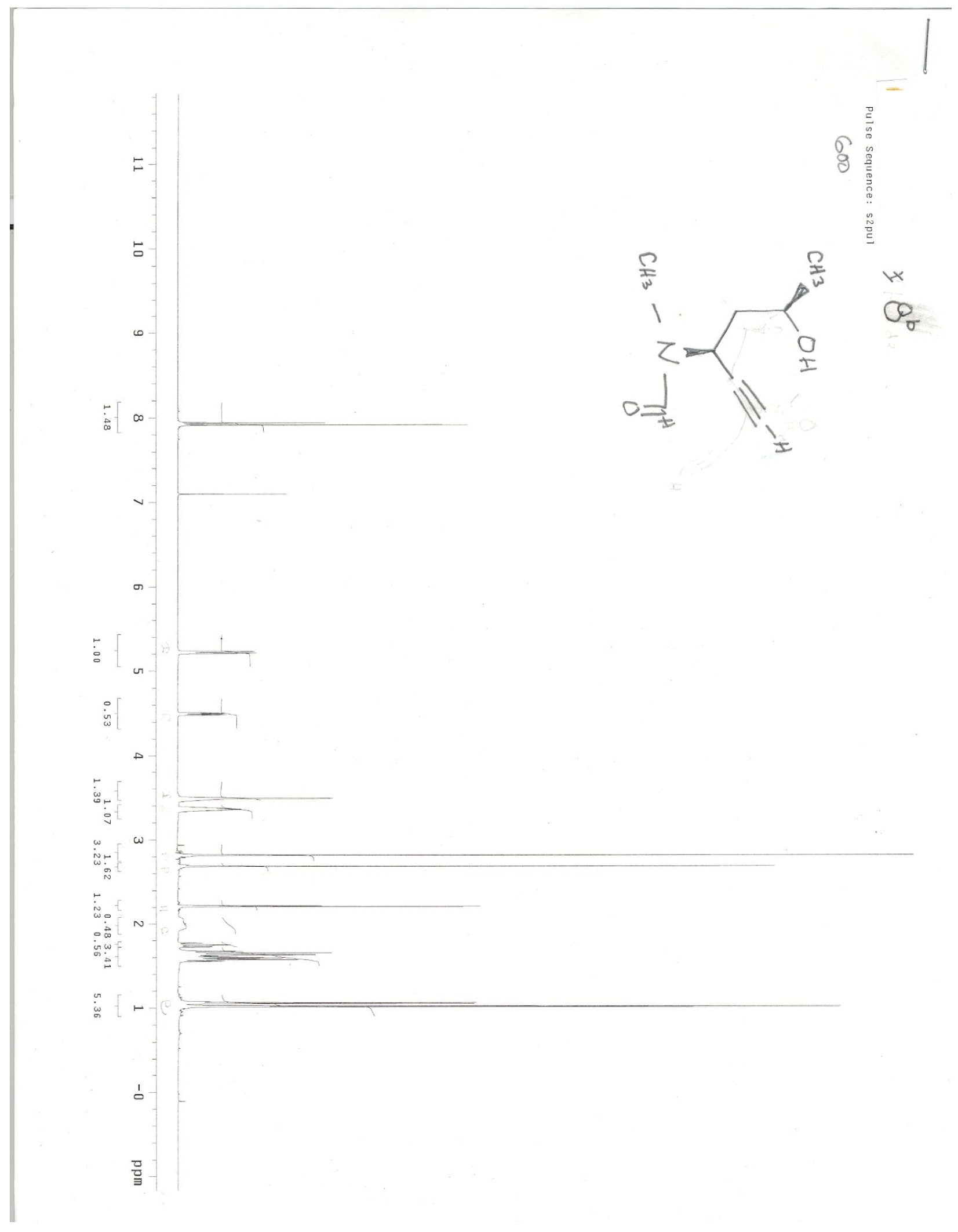




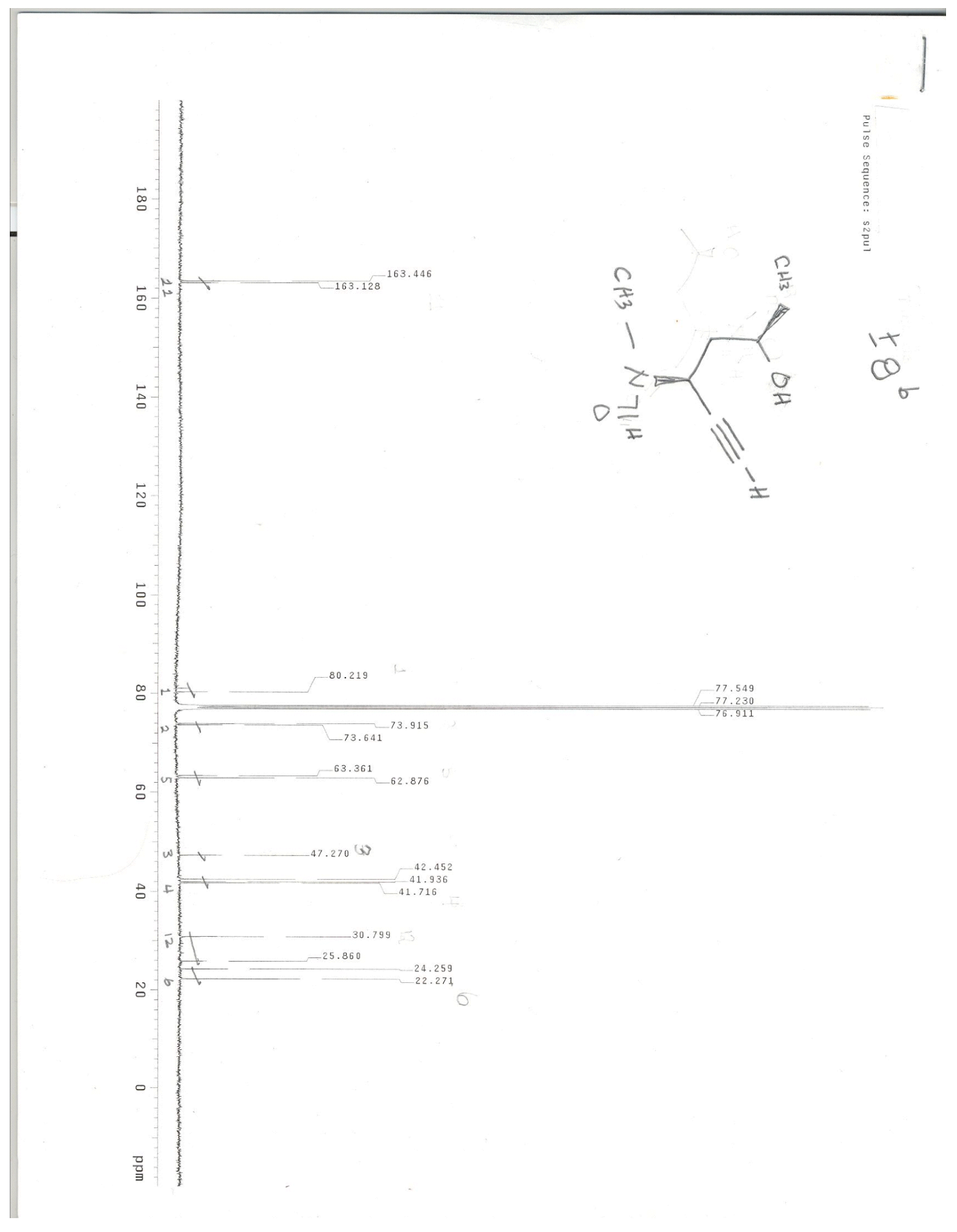




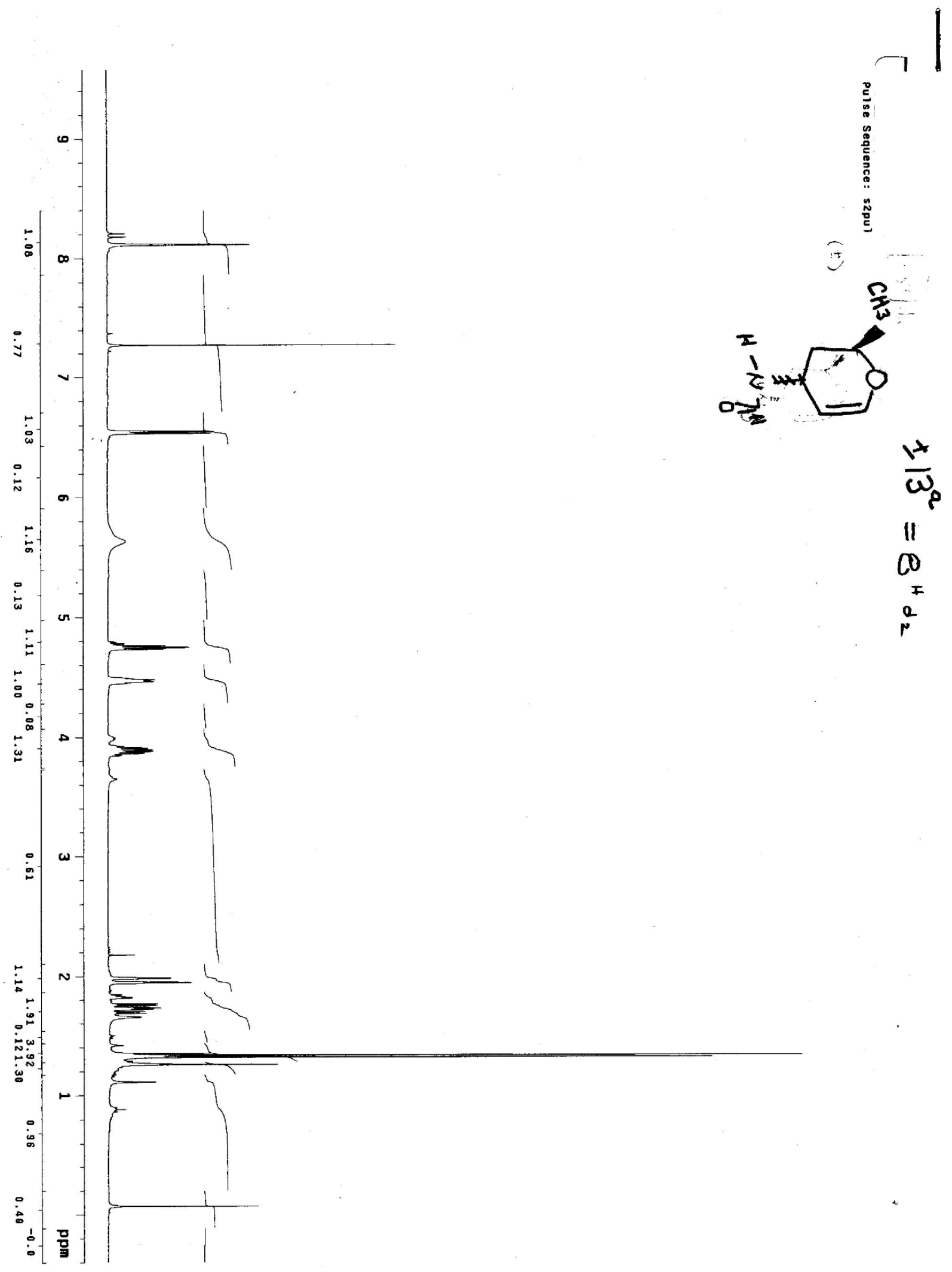




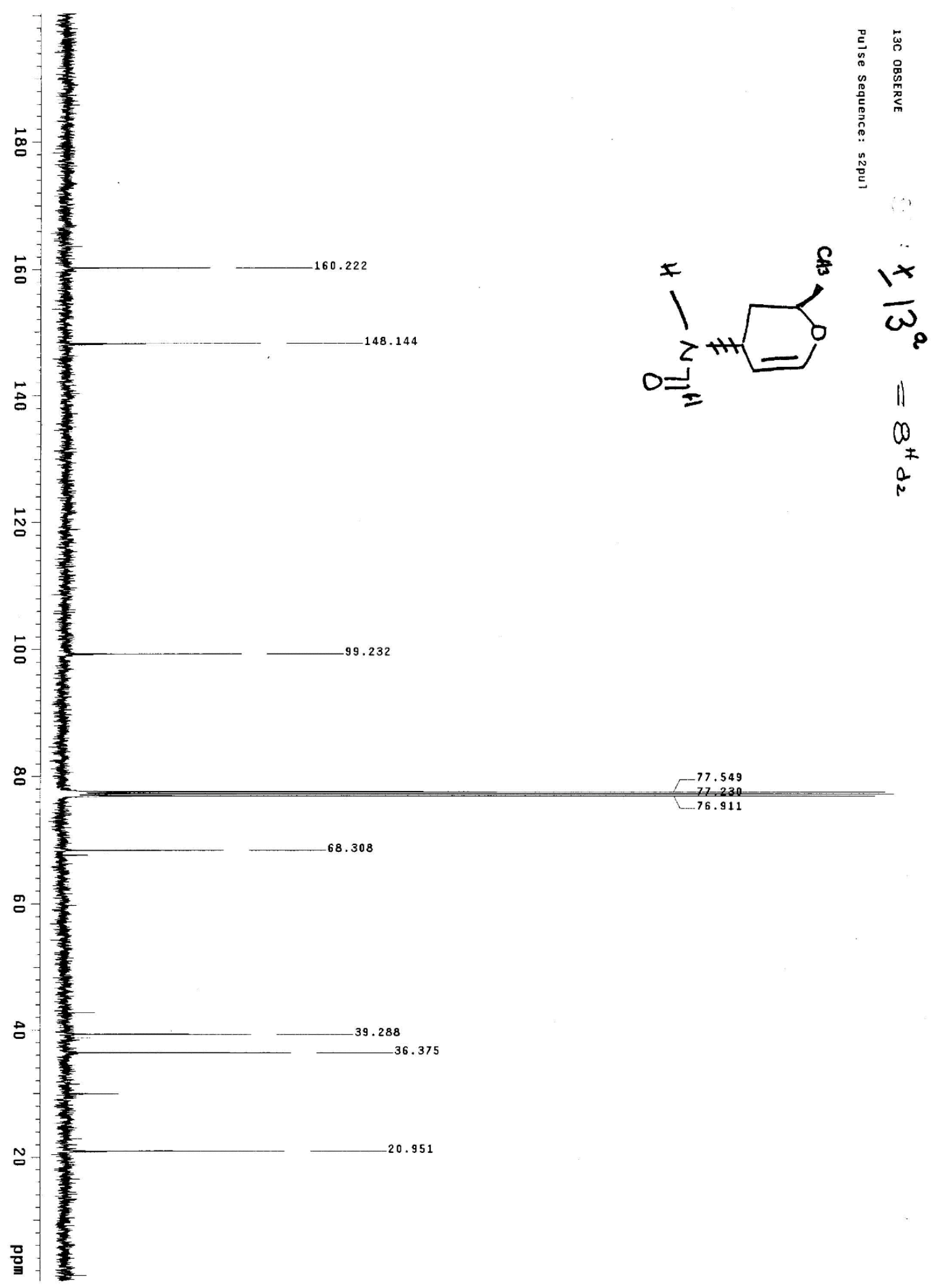



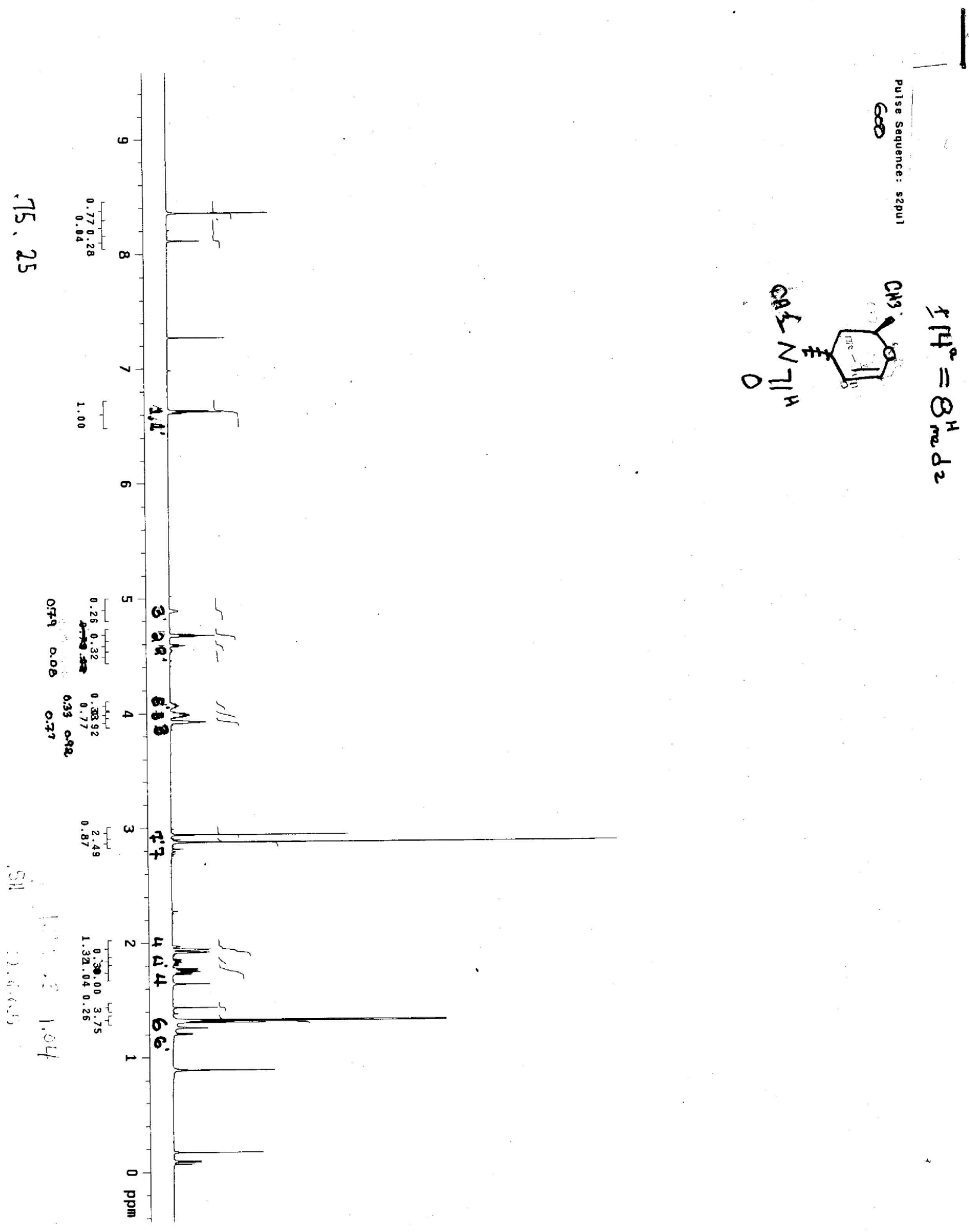


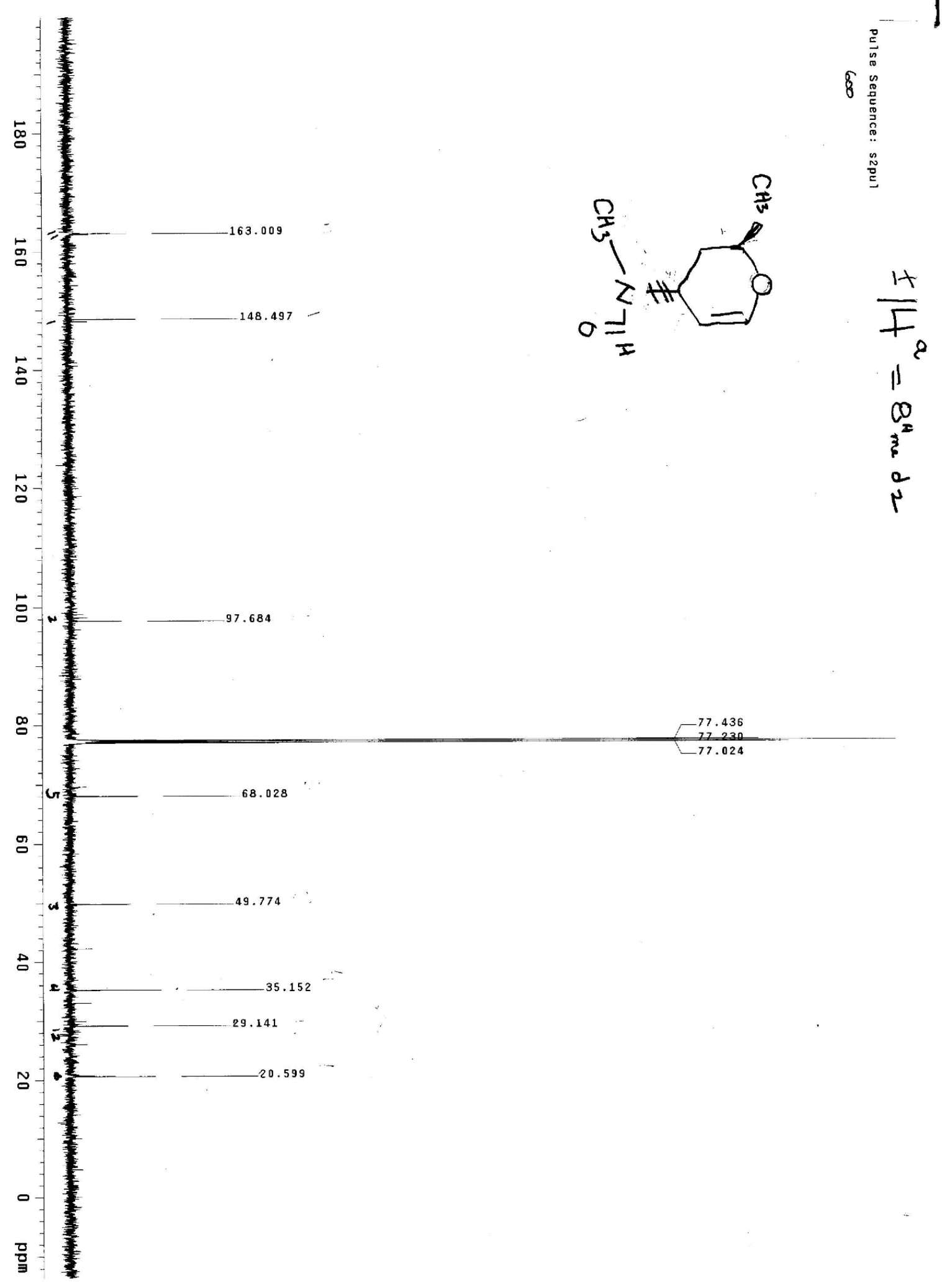




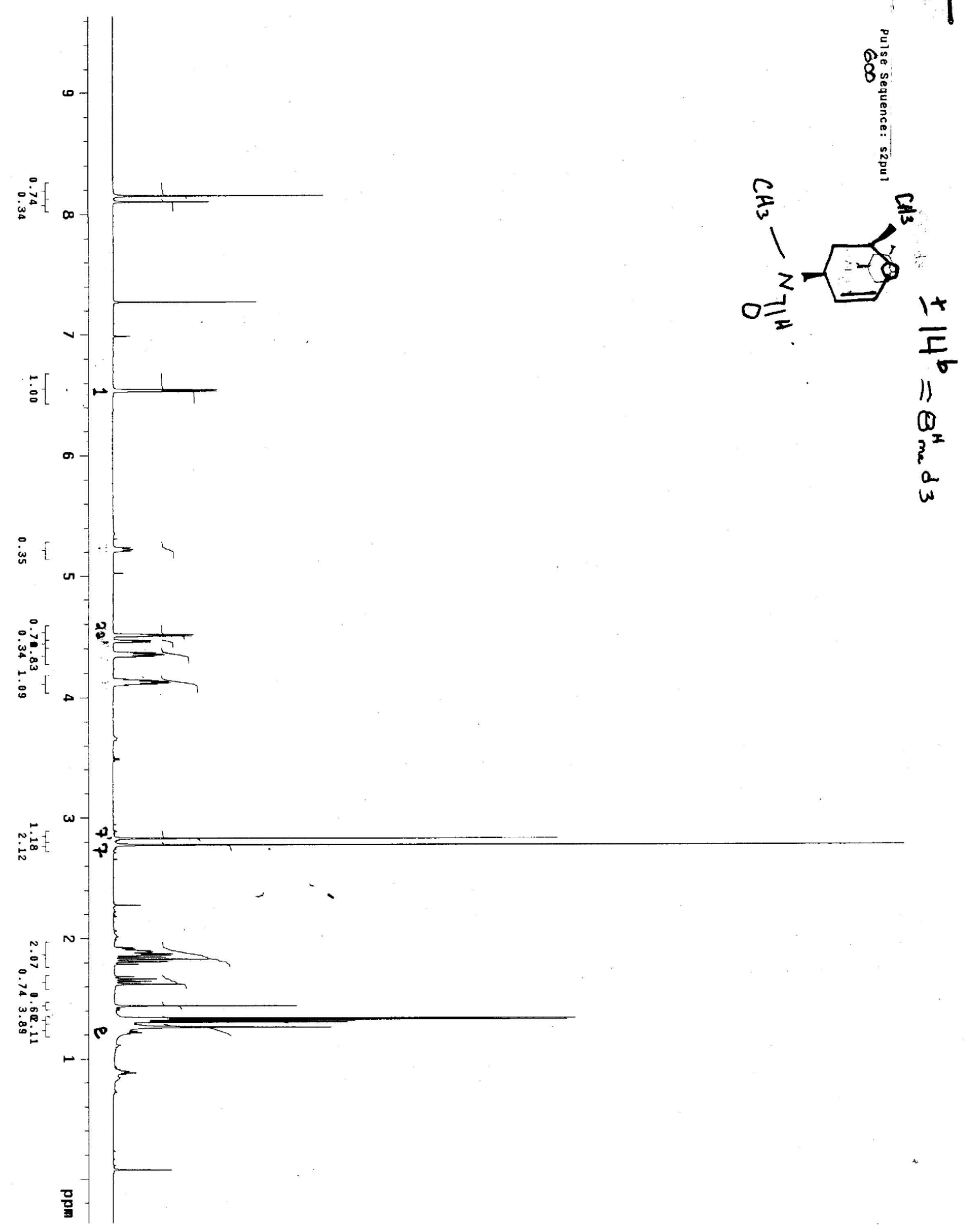




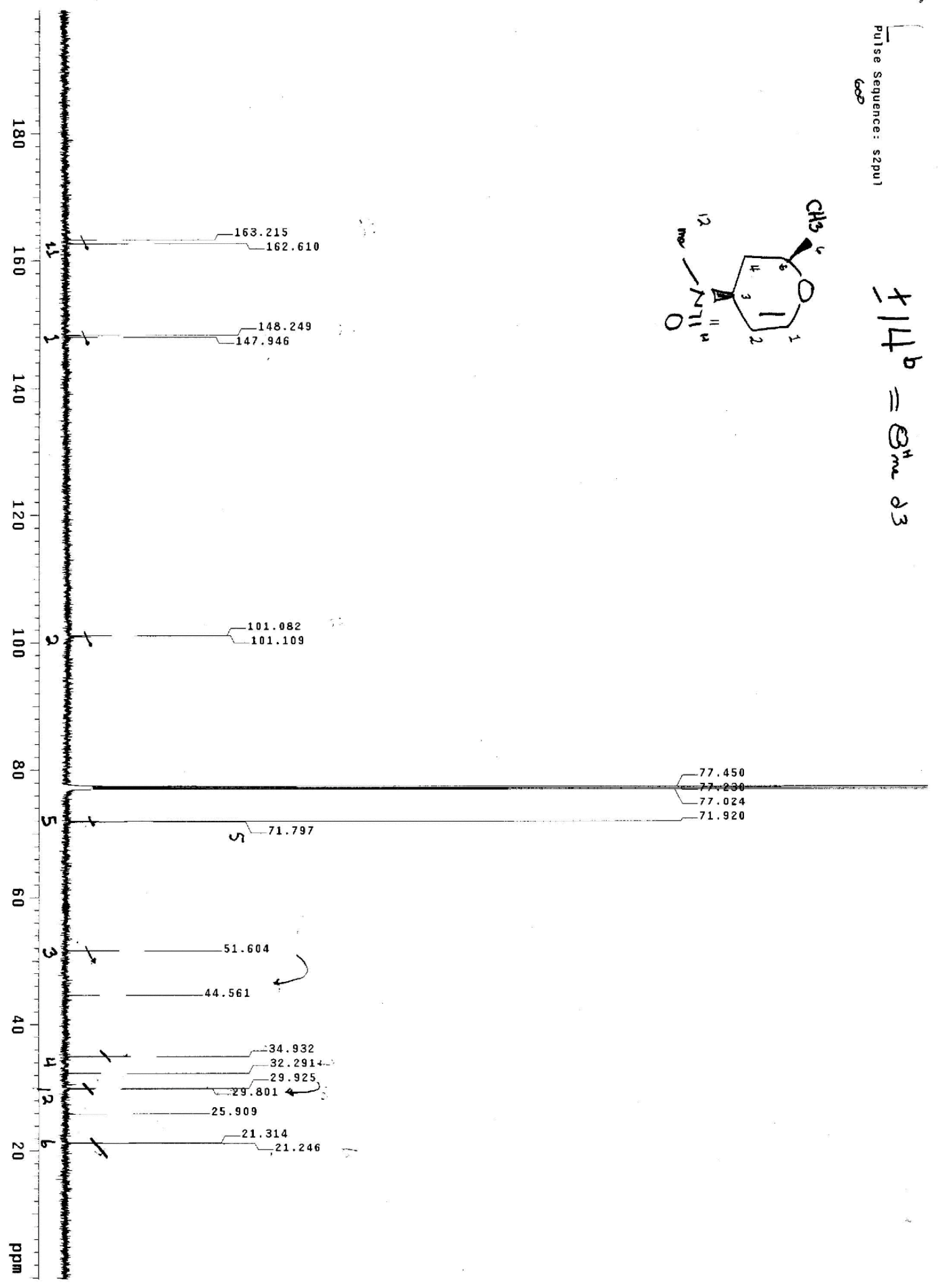




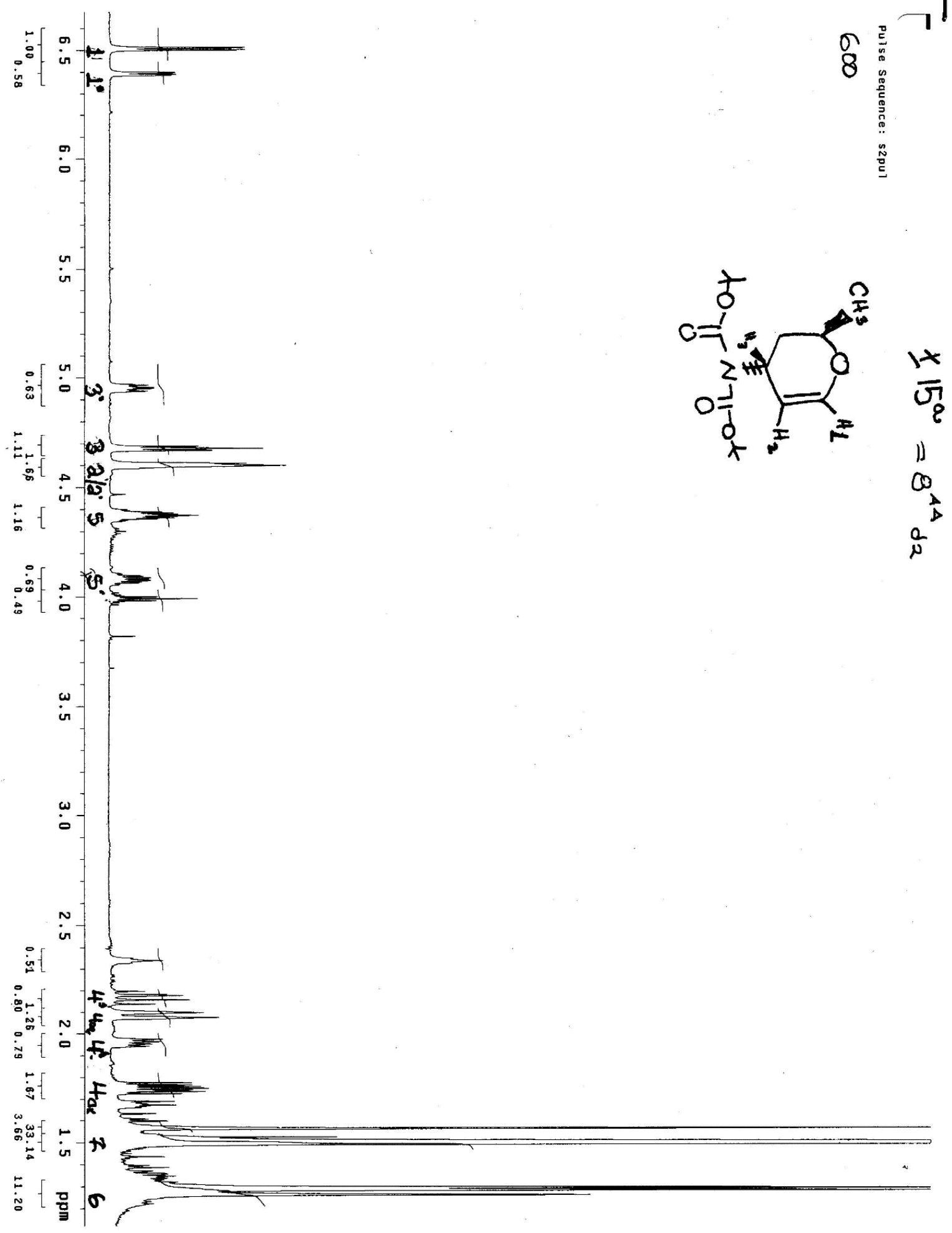




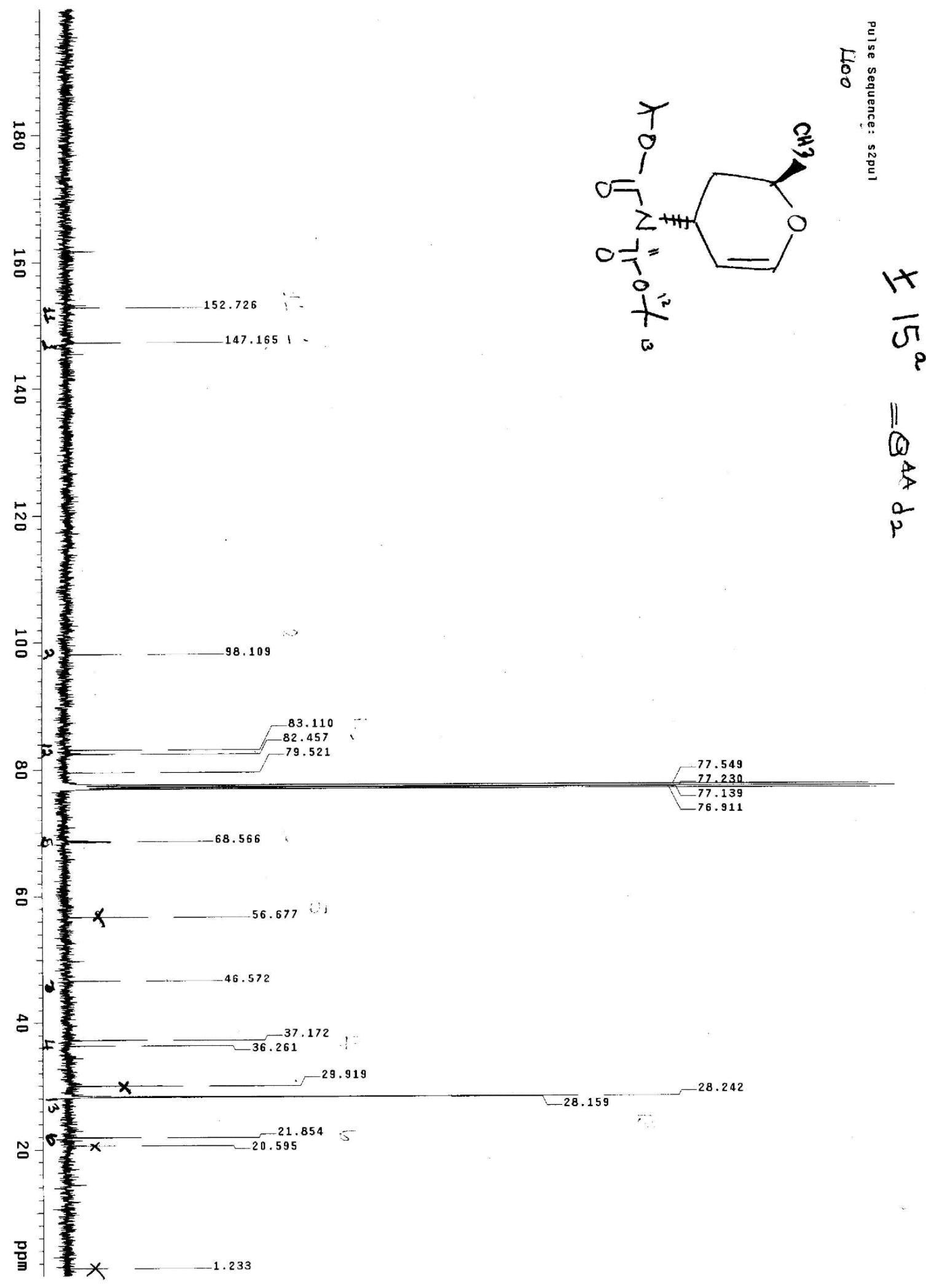




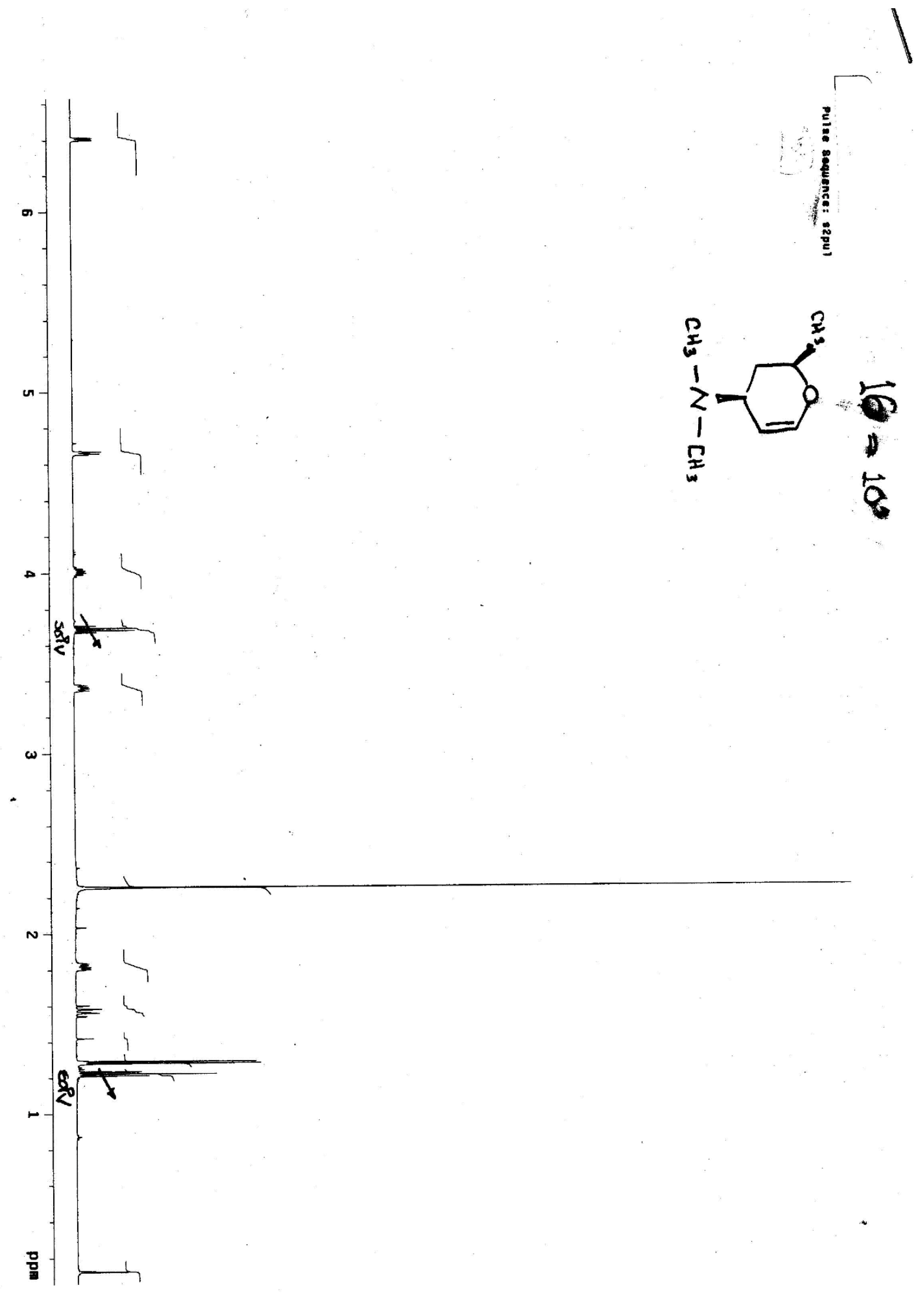




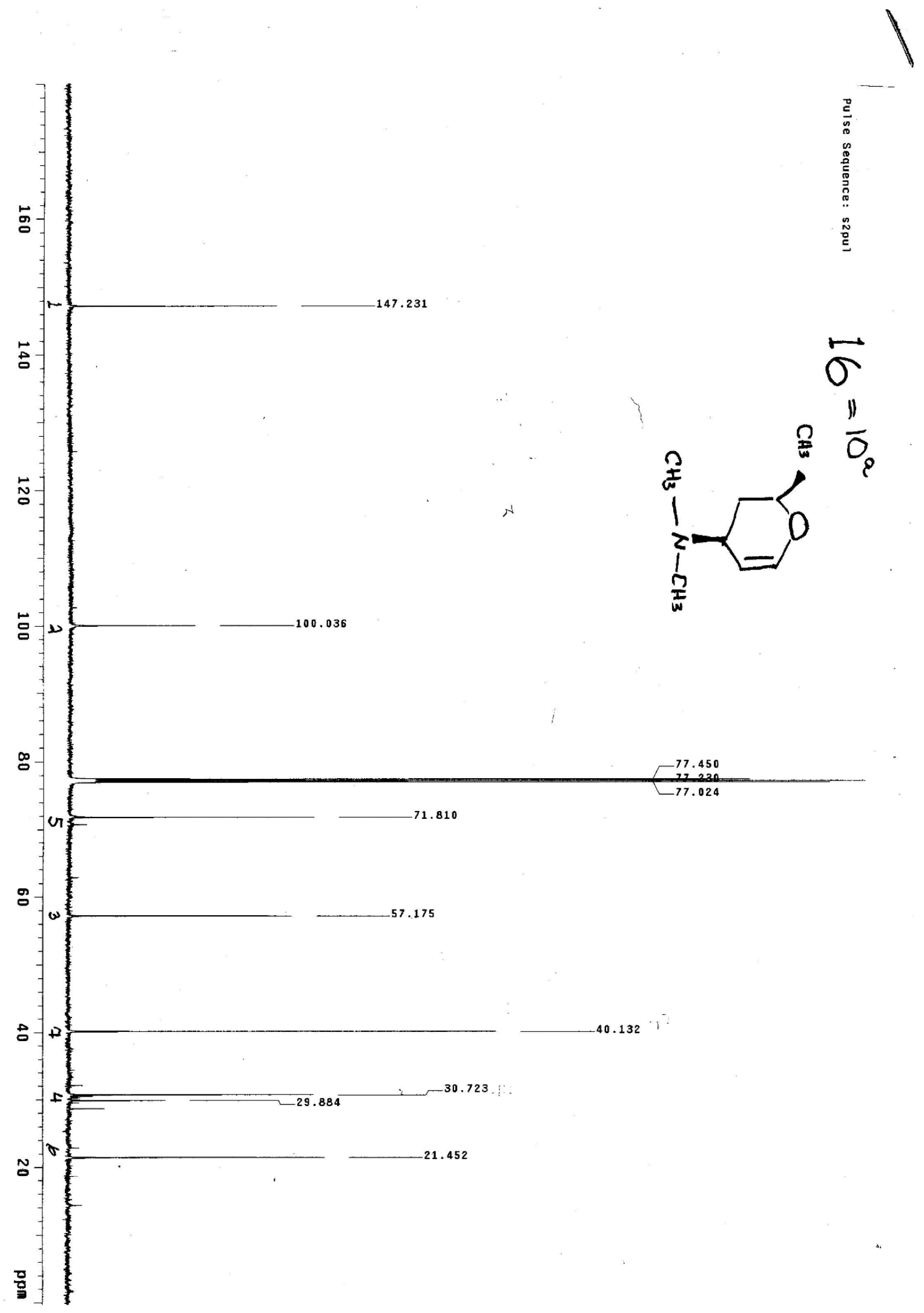




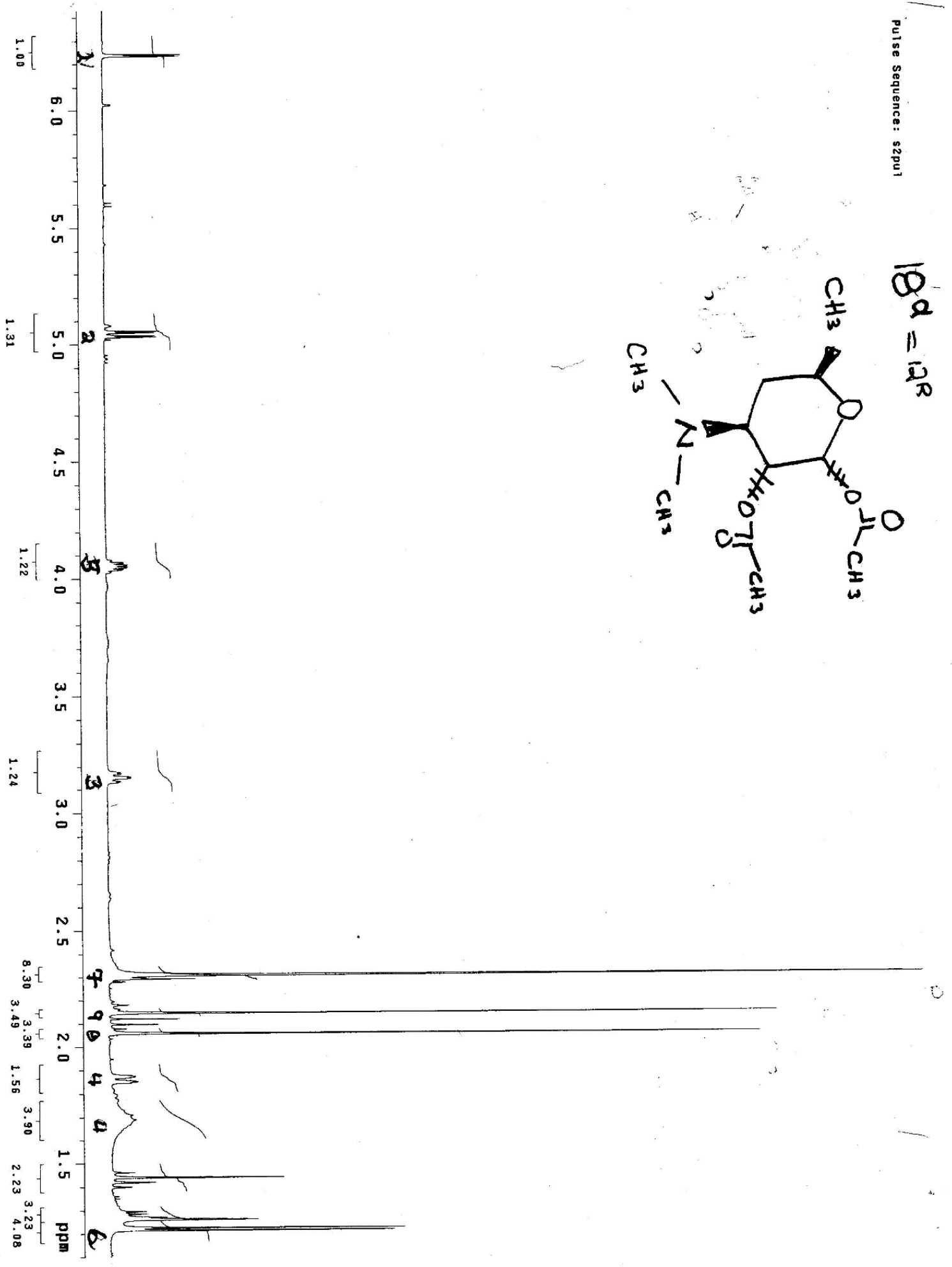




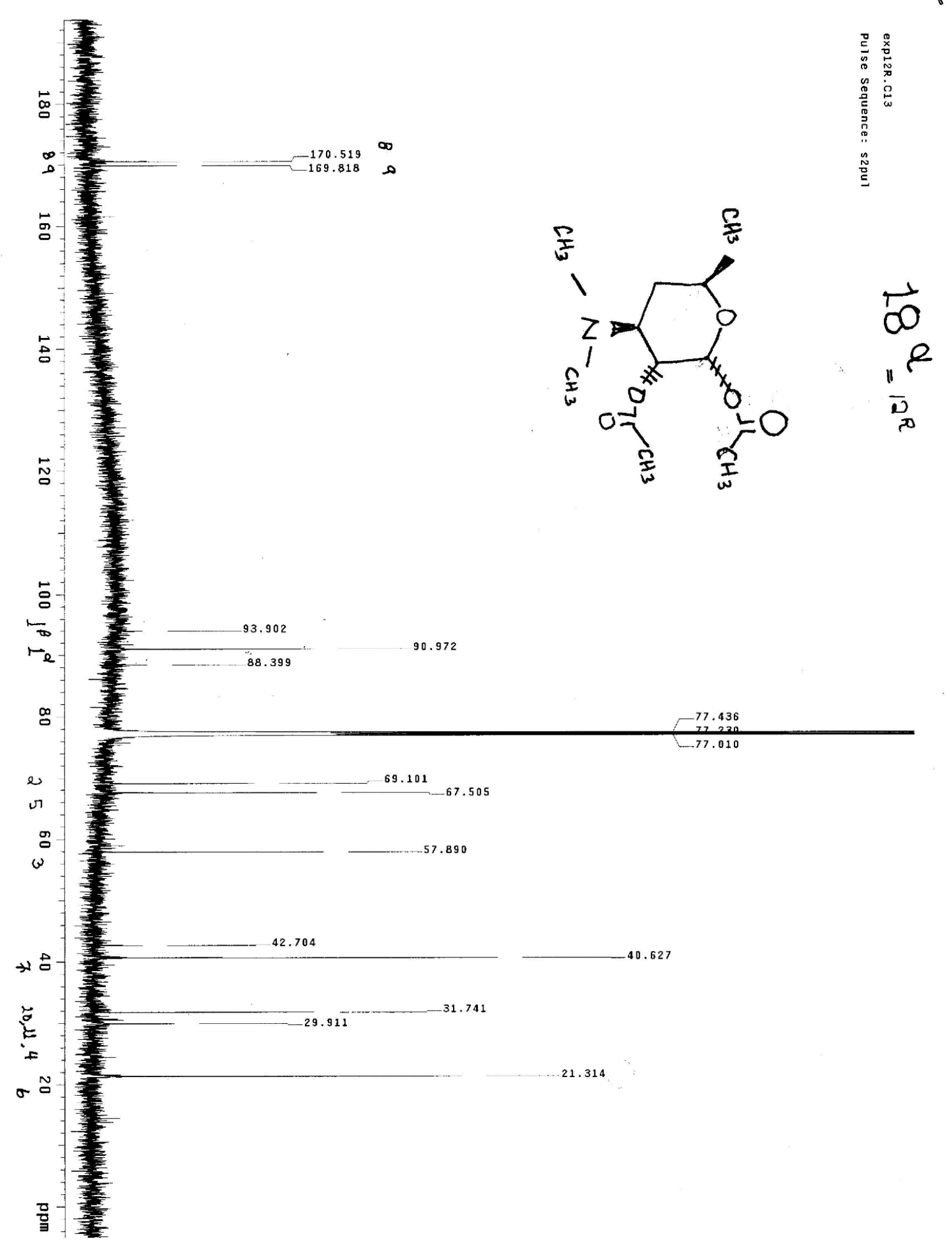




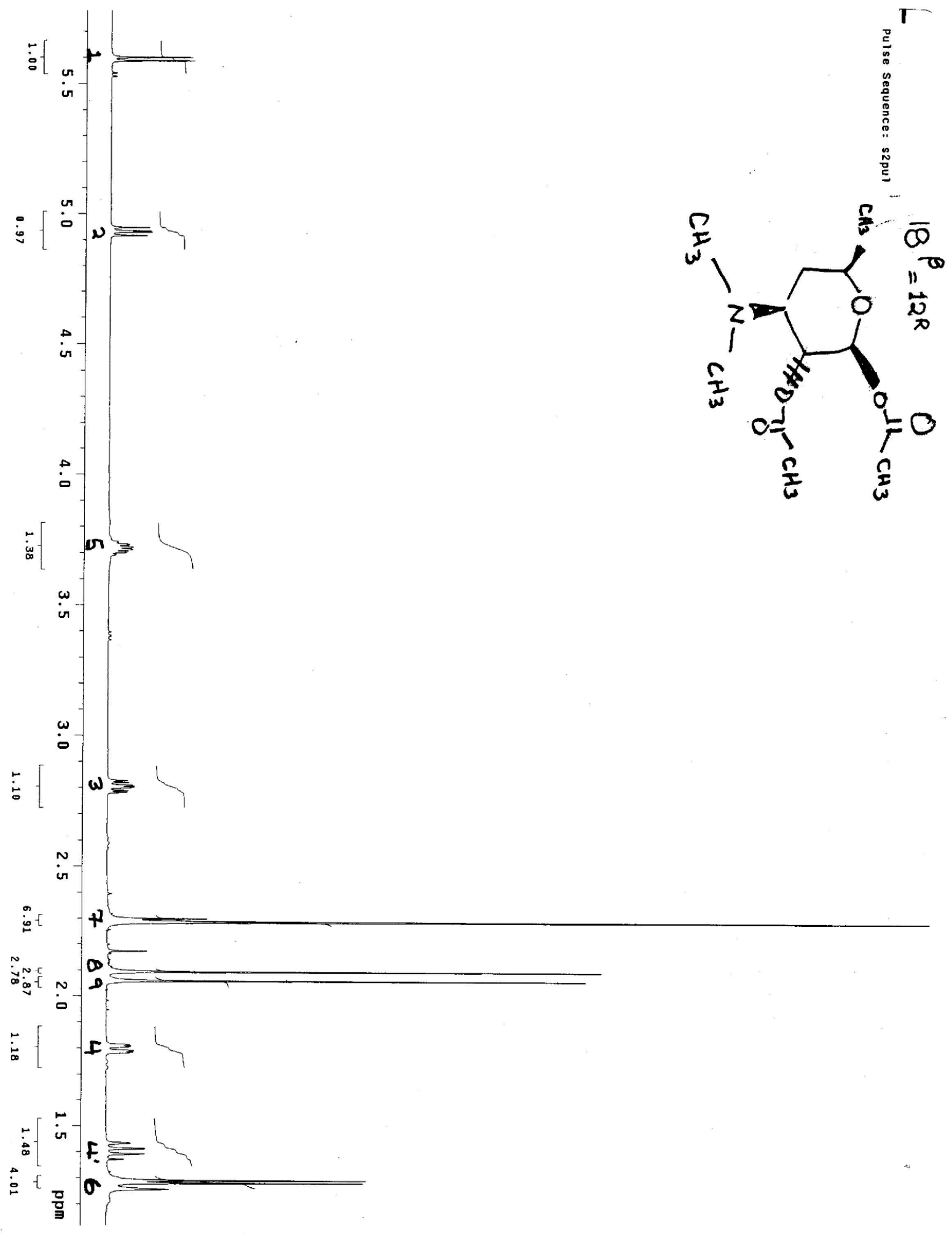




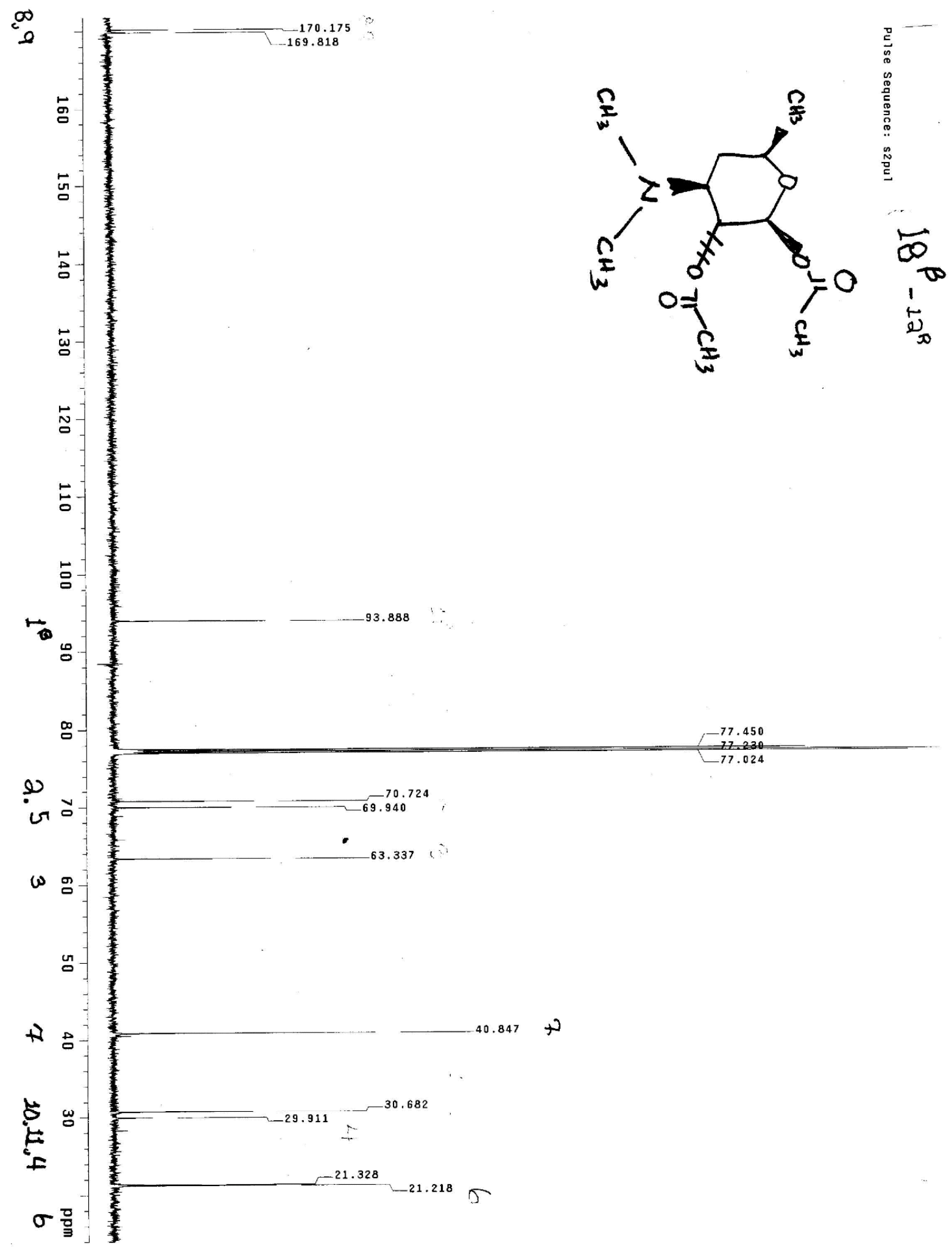

